\title{
pdCSM-PPI: Using Graph-Based Signatures to Identify Protein-Protein Interaction Inhibitors
}

\author{
Carlos H. M. Rodrigues ${ }^{1,2,3}$, Douglas E. V. Pires ${ }^{1,2,3,4^{*}}$, David B. Ascher ${ }^{1,2,3^{*}}$ \\ ${ }^{1}$ Systems and Computational Biology, Bio21 Institute, University of Melbourne, Parkville 3052, Victoria, Australia \\ ${ }^{2}$ Computational Biology and Clinical Informatics, Baker Heart and Diabetes Institute, Melbourne 3004, Victoria, Australia \\ ${ }^{3}$ School of Chemistry and Molecular Biosciences, The University of Queensland, Brisbane, 4072 Australia \\ ${ }^{4}$ School of Computing and Information Systems, University of Melbourne, Parkville 3052, Victoria, Australia \\ *To whom correspondence should be addressed D.B.A. Tel: +61 90354794; Email: david.ascher@unimelb.edu.au. \\ Correspondence may also be addressed to D.E.V.P. douglas.pires@unimelb.edu.au.
}

\section{TABLES}

Table S1. Feature importance for predictive model of PPI inhibitors targeting the Bcl2/Bak complex.

\begin{tabular}{|l|l|r|}
\hline Feature & Description & Importance \\
\hline Aromatic_Count & Number of atoms in aromatic rings & 0.105 \\
\hline Hydrophobe:Hydrophobe-4.00 & $\begin{array}{l}\text { Number of pairs of hydrophobe atoms within 4 } \\
\text { bonds }\end{array}$ & 0.055 \\
\hline Chi2n & Molecular connectivity index & 0.052 \\
\hline fr_sulfide & Number of thioether & 0.051 \\
\hline SlogP_VSA8 & $\begin{array}{l}\text { MOE-type descriptors, LogP and surface area } \\
\text { contributions }\end{array}$ & 0.046 \\
\hline fr_Ar_N & Number of aromatic nitrogens & 0.042 \\
\hline fr_bicyclic & Number of bicyclic structures & 0.042 \\
\hline SMR_VSA10 & $\begin{array}{l}\text { MOE-type descriptors, molar refractivity and } \\
\text { surface area contributions }\end{array}$ \\
\hline Acceptor:Hydrophobe-3.00 & $\begin{array}{l}\text { Number of pairs of acceptor-hydrophobe atoms } \\
\text { within 3 bonds }\end{array}$ & 0.037 \\
\hline VSA_EState8 & $\begin{array}{l}\text { MOE-type descriptors, surface area contributions } \\
\text { and EState indices }\end{array}$ & 0.028 \\
\hline SlogP_VSA12 & $\begin{array}{l}\text { MOE-type descriptors, LogP and surface area } \\
\text { contributions }\end{array}$ & 0.027 \\
\hline SMR_VSA9 & $\begin{array}{l}\text { MOE-type descriptors, LogP and surface area } \\
\text { contributions }\end{array}$ & 0.026 \\
\hline SlogP_VSA10 & $\begin{array}{l}\text { MOE-type descriptors, LogP and surface area } \\
\text { contributions }\end{array}$ \\
\hline Donor:Donor-3.00 & $\begin{array}{l}\text { Number of pairs of hydrophobe atoms within 3 } \\
\text { bonds }\end{array}$ & 0.022 \\
\hline
\end{tabular}




\begin{tabular}{|c|c|c|}
\hline fr_thiazole & Number of thiazole rings & 0.021 \\
\hline Donor_Count & Number hydrogen bond donors & 0.020 \\
\hline SMR_VSA5 & $\begin{array}{l}\text { MOE-type descriptors, molar refractivity and } \\
\text { surface area contributions }\end{array}$ & 0.019 \\
\hline Acceptor:Hydrophobe-2.00 & $\begin{array}{l}\text { Number of pairs of acceptor-hydrophobe atoms } \\
\text { within } 2 \text { bonds }\end{array}$ & 0.019 \\
\hline Donor:Hydrophobe-1.00 & $\begin{array}{l}\text { Number of pairs of donor-hydrophobe atoms within } \\
1 \text { bond }\end{array}$ & 0.018 \\
\hline Donor:Hydrophobe-3.00 & $\begin{array}{l}\text { Number of pairs of donor-hydrophobe atoms within } \\
3 \text { bond }\end{array}$ & 0.018 \\
\hline fr_pyridine & Number of pyridine rings & 0.017 \\
\hline fr_phenol & $\begin{array}{l}\text { Number of phenolic } \mathrm{OH} \text { excluding ortho } \\
\text { intramolecular Hbond substituents }\end{array}$ & 0.017 \\
\hline PEOE_VSA9 & $\begin{array}{l}\text { MOE-type descriptors, partial charges and surface } \\
\text { area contributions }\end{array}$ & 0.016 \\
\hline SlogP_VSA7 & $\begin{array}{l}\text { MOE-type descriptors, LogP and surface area } \\
\text { contributions }\end{array}$ & 0.016 \\
\hline Donor:Donor-6.00 & Number of pairs of hydrogen donor within 6 bonds & 0.016 \\
\hline Poslonizable_Count & Number of Poslonizable atoms & 0.015 \\
\hline fr_Ar_OH & Number of aromatic hydroxyl groups & 0.014 \\
\hline Donor:Donor-1.00 & $\begin{array}{l}\text { Number of pairs of hydrogen donor atoms within } 1 \\
\text { bond }\end{array}$ & 0.012 \\
\hline Aromatic:Neglonizable-4.00 & $\begin{array}{l}\text { Number of pairs of aromatic-neglonizable atoms } \\
\text { within } 4 \text { bonds }\end{array}$ & 0.011 \\
\hline fr_Imine & Number of Imines & 0.009 \\
\hline fr_NH2 & Number of Primary amines & 0.009 \\
\hline Hydrophobe:Poslonizable-4.00 & $\begin{array}{l}\text { Number of pairs of hydrophobe-neglonizable atoms } \\
\text { within } 4 \text { bonds }\end{array}$ & 0.008 \\
\hline Acceptor:Poslonizable-1.00 & $\begin{array}{l}\text { Number of pairs of acceptor-poslonizable atoms } \\
\text { within } 1 \text { bond }\end{array}$ & 0.008 \\
\hline Acceptor:Poslonizable-4.00 & $\begin{array}{l}\text { Number of pairs of acceptor-poslonizable atoms } \\
\text { within } 4 \text { bonds }\end{array}$ & 0.008 \\
\hline Neglonizable:Neglonizable-1.00 & $\begin{array}{l}\text { Number of pairs of neglonizable-neglonizable } \\
\text { atoms within } 1 \text { bond }\end{array}$ & 0.008 \\
\hline Poslonizable:Poslonizable-4.00 & $\begin{array}{l}\text { Number of pairs of poslonizable-poslonizable } \\
\text { atoms within } 4 \text { bonds }\end{array}$ & 0.007 \\
\hline fr_alkyl_halide & Number of alkyl halides & 0.007 \\
\hline Neglonizable:Neglonizable-4.00 & $\begin{array}{l}\text { Number of pairs of neglonizable-neglonizable } \\
\text { atoms within } 4 \text { bonds }\end{array}$ & 0.007 \\
\hline fr_unbrch_alkane & $\begin{array}{l}\text { Number of unbranched alkanes of at least } 4 \\
\text { members (excludes halogenated alkanes) }\end{array}$ & 0.007 \\
\hline Neglonizable:Neglonizable-2.00 & $\begin{array}{l}\text { Number of pairs of neglonizable-neglonizable } \\
\text { atoms within } 2 \text { bonds }\end{array}$ & 0.007 \\
\hline Neglonizable:Neglonizable-5.00 & $\begin{array}{l}\text { Number of pairs of neglonizable-neglonizable } \\
\text { atoms within } 5 \text { bonds }\end{array}$ & 0.006 \\
\hline Donor:Poslonizable-3.00 & $\begin{array}{l}\text { Number of pairs of donor-poslonizable atoms } \\
\text { within } 3 \text { bonds }\end{array}$ & 0.006 \\
\hline
\end{tabular}




\begin{tabular}{|l|l|r|}
\hline Hydrophobe:Poslonizable-3.00 & $\begin{array}{l}\text { Number of pairs of hydrophobe-poslonizable atoms } \\
\text { within 3 bonds }\end{array}$ & 0.006 \\
\hline Poslonizable:Poslonizable-3.00 & $\begin{array}{l}\text { Number of pairs of poslonizable-poslonizable } \\
\text { atoms within 3 bonds }\end{array}$ & 0.006 \\
\hline Hydrophobe:Neglonizable-1.00 & $\begin{array}{l}\text { Number of pairs of hydrophobe-neglonizable atoms } \\
\text { within 1 bond }\end{array}$ & 0.005 \\
\hline Hydrophobe:Neglonizable-4.00 & $\begin{array}{l}\text { Number of pairs of hydrophobe-neglonizable atoms } \\
\text { within 4 bonds }\end{array}$ & 0.005 \\
\hline fr_ketone & Number of ketones & 0.005 \\
\hline Poslonizable:Poslonizable-2.00 & $\begin{array}{l}\text { Number of pairs of poslonizable-poslonizable } \\
\text { atoms within 2 bonds }\end{array}$ & 0.005 \\
\hline Hydrophobe:Neglonizable-2.00 & $\begin{array}{l}\text { Number of pairs of hydrophobe-neglonizable atoms } \\
\text { within 2 bonds }\end{array}$ & 0.005 \\
\hline Acceptor:Neglonizable-1.00 & $\begin{array}{l}\text { Number of pairs of acceptor-neglonizable atoms } \\
\text { within 1 bond }\end{array}$ & 0.004 \\
\hline fr_priamide & Number of primary amides & 0.004 \\
\hline Donor:Poslonizable-1.00 & $\begin{array}{l}\text { Number of pairs of donor-poslonizable atoms } \\
\text { within 1 bond }\end{array}$ & 0.004 \\
\hline Acceptor:Neglonizable-4.00 & $\begin{array}{l}\text { Number of pairs of acceptor-neglonizable atoms } \\
\text { within 4 bonds }\end{array}$ & 0.004 \\
\hline Acceptor:Neglonizable-2.00 & $\begin{array}{l}\text { Number of pairs of acceptor-neglonizable atoms } \\
\text { within 2 bonds }\end{array}$ & 0.004 \\
\hline Donor:Neglonizable-5.00 & $\begin{array}{l}\text { Number of pairs of acceptor-neglonizable atoms } \\
\text { within 5 bonds }\end{array}$ & 0.004 \\
\hline Tox_2 & {$[$ CH]=[CH]O } & 0.004 \\
\hline fr_thiophene & Number of thiophene rings \\
\hline Donor:Neglonizable-1.00 & $\begin{array}{l}\text { Number of pairs of donor-neglonizable atoms } \\
\text { within 1 bond }\end{array}$ & $\begin{array}{l}\text { Number of pairs of hydrophobe-poslonizable atoms } \\
\text { within 1 bond }\end{array}$ \\
\hline Hydrophobe:Poslonizable-1.00 & $\begin{array}{l}\text { Number of pairs of donor-poslonizable atoms } \\
\text { within 3 bonds }\end{array}$ & 0.003 \\
\hline Donor:Neglonizable-3.00 & Number of guanidine groups \\
\hline fr_lactone & Number of cyclic esters (lactones) \\
\hline
\end{tabular}

Table S2. Feature importance for predictive model of PPI inhibitors targeting the Bromodomain/Histone complex.

\begin{tabular}{|l|l|r|}
\hline Feature & Description & Importance \\
\hline Donor:Hydrophobe-4.00 & $\begin{array}{l}\text { Number of pairs of donor-hydrophobe atoms } \\
\text { within 4 bonds }\end{array}$ & 0.044 \\
\hline Kappa2 & Molecular shape index & 0.043 \\
\hline fr_aryl_methyl & Number of aryl methyl sites for hydroxylation & 0.041 \\
\hline Donor:Hydrophobe-1.00 & $\begin{array}{l}\text { Number of pairs of donor-hydrophobe atoms } \\
\text { within 1 bond }\end{array}$ & 0.037 \\
\hline BalabanJ & Balaban's connectivity topological index & 0.033 \\
\hline fr_Al_COO & Number of aliphatic carboxylic acids & 0.024 \\
\hline
\end{tabular}




\begin{tabular}{|c|c|c|}
\hline fr_halogen & Number of halogens & 0.024 \\
\hline VSA_EState10 & $\begin{array}{l}\text { MOE-type descriptors surface area contributions } \\
\text { and EState indices }\end{array}$ & 0.024 \\
\hline Chi1v & Molecular connectivity index & 0.023 \\
\hline SMR_VSA5 & $\begin{array}{l}\text { MOE-type descriptors, molar refractivity and } \\
\text { surface area contributions }\end{array}$ & 0.022 \\
\hline fr_aniline & Number of anilines & 0.022 \\
\hline Aromatic:Aromatic-5.00 & $\begin{array}{l}\text { Number of pairs of aromatic-aromatic atoms } \\
\text { within } 5 \text { bonds }\end{array}$ & 0.021 \\
\hline Aromatic:Donor-3.00 & $\begin{array}{l}\text { Number of pairs of aromatic-donor atoms within } 3 \\
\text { bonds }\end{array}$ & 0.021 \\
\hline Hydrophobe:Hydrophobe- 4.00 & $\begin{array}{l}\text { Number of pairs of hydrophobe-hydrophobe } \\
\text { atoms within } 4 \text { bonds }\end{array}$ & 0.020 \\
\hline fr_benzene & Number of benzene rings & 0.020 \\
\hline VSA_EState8 & $\begin{array}{l}\text { MOE-type descriptors surface area contributions } \\
\text { and EState indices }\end{array}$ & 0.018 \\
\hline SlogP_VSA5 & $\begin{array}{l}\text { MOE-type descriptors, LogP and surface area } \\
\text { contributions }\end{array}$ & 0.018 \\
\hline PEOE_VSA2 & $\begin{array}{l}\text { MOE-type descriptors, partial charges and } \\
\text { surface area contributions }\end{array}$ & 0.017 \\
\hline Aromatic:Hydrophobe-1.00 & $\begin{array}{l}\text { Number of pairs of aromatic-hydrophobe atoms } \\
\text { within } 1 \text { bond }\end{array}$ & 0.017 \\
\hline Acceptor:Aromatic-5.00 & $\begin{array}{l}\text { Number of pairs of acceptor-aromatic atoms } \\
\text { within } 5 \text { bonds }\end{array}$ & 0.017 \\
\hline SMR_VSA6 & $\begin{array}{l}\text { MOE-type descriptors, molar refractivity and } \\
\text { surface area contributions }\end{array}$ & 0.017 \\
\hline Aromatic:Hydrophobe-5.00 & $\begin{array}{l}\text { Number of pairs of aromatic-hydrophobe atoms } \\
\text { within } 5 \text { bonds }\end{array}$ & 0.017 \\
\hline Aromatic_Count & Number of atoms in aromatic rings & 0.016 \\
\hline Hydrophobe:Hydrophobe- 1.00 & $\begin{array}{l}\text { Number of pairs of hydrophobe-hydrophobe } \\
\text { atoms within } 1 \text { bond }\end{array}$ & 0.016 \\
\hline SlogP_VSA10 & $\begin{array}{l}\text { MOE-type descriptors, LogP and surface area } \\
\text { contributions }\end{array}$ & 0.016 \\
\hline SMR_VSA10 & $\begin{array}{l}\text { MOE-type descriptors, molar refractivity and } \\
\text { surface area contributions }\end{array}$ & 0.015 \\
\hline fr_NHO & Number of Tertiary amines & 0.015 \\
\hline Donor:Donor-6.00 & $\begin{array}{l}\text { Number of pairs of donor-donor atoms within } 6 \\
\text { bonds }\end{array}$ & 0.015 \\
\hline SMR_VSA4 & $\begin{array}{l}\text { MOE-type descriptors, molar refractivity and } \\
\text { surface area contributions }\end{array}$ & 0.015 \\
\hline Acceptor:Aromatic-4.00 & $\begin{array}{l}\text { Number of pairs of acceptor-aromatic atoms } \\
\text { within } 4 \text { bonds }\end{array}$ & 0.015 \\
\hline PEOE_VSA7 & $\begin{array}{l}\text { MOE-type descriptors, partial charges and } \\
\text { surface area contributions }\end{array}$ & 0.014 \\
\hline Acceptor:Aromatic-2.00 & $\begin{array}{l}\text { Number of pairs of acceptor-aromatic atoms } \\
\text { within } 2 \text { bonds }\end{array}$ & 0.014 \\
\hline Donor:Donor-5.00 & $\begin{array}{l}\text { Number of pairs of donor-donor atoms within } 5 \\
\text { bonds }\end{array}$ & 0.014 \\
\hline
\end{tabular}




\begin{tabular}{|c|c|c|}
\hline PEOE_VSA3 & $\begin{array}{l}\text { MOE-type descriptors, partial charges and } \\
\text { surface area contributions }\end{array}$ & 0.014 \\
\hline PEOE_VSA9 & $\begin{array}{l}\text { MOE-type descriptors, partial charges and } \\
\text { surface area contributions }\end{array}$ & 0.013 \\
\hline SlogP_VSA1 & $\begin{array}{l}\text { MOE-type descriptors, LogP and surface area } \\
\text { contributions }\end{array}$ & 0.013 \\
\hline fr_Nhpyrrole & Number of $\mathrm{H}$-pyrrole nitrogens & 0.013 \\
\hline Aromatic:Donor-1.00 & $\begin{array}{l}\text { Number of pairs of aromatic-donor atoms within } 1 \\
\text { bond }\end{array}$ & 0.013 \\
\hline PEOE_VSA11 & $\begin{array}{l}\text { MOE-type descriptors, partial charges and } \\
\text { surface area contributions }\end{array}$ & 0.012 \\
\hline PEOE_VSA4 & $\begin{array}{l}\text { MOE-type descriptors, partial charges and } \\
\text { surface area contributions }\end{array}$ & 0.012 \\
\hline Acceptor:Acceptor-1.00 & $\begin{array}{l}\text { Number of pairs of acceptor-acceptor atoms } \\
\text { within } 1 \text { bond }\end{array}$ & 0.012 \\
\hline Acceptor:Acceptor-5.00 & $\begin{array}{l}\text { Number of pairs of acceptor-acceptor atoms } \\
\text { within } 5 \text { bonds }\end{array}$ & 0.012 \\
\hline Neglonizable_Count & Number of neglonizable atoms & 0.011 \\
\hline Poslonizable_Count & Number of poslonizable atoms & 0.010 \\
\hline Donor:Donor-4.00 & $\begin{array}{l}\text { Number of pairs of donor-donor atoms within } 4 \\
\text { bonds }\end{array}$ & 0.010 \\
\hline PEOE_VSA5 & $\begin{array}{l}\text { MOE-type descriptors, partial charges and } \\
\text { surface area contributions }\end{array}$ & 0.009 \\
\hline Aromatic:Poslonizable-6.00 & $\begin{array}{l}\text { Number of pairs of aromatic-poslonizable atoms } \\
\text { within } 6 \text { bonds }\end{array}$ & 0.008 \\
\hline Donor:Donor-3.00 & $\begin{array}{l}\text { Number of pairs of donor-donor atoms within } 3 \\
\text { bonds }\end{array}$ & 0.008 \\
\hline fr_Imine & Number of Imines & 0.007 \\
\hline fr_Ar_OH & Number of aromatic hydroxyl groups & 0.007 \\
\hline fr_ketone & Number of ketones & 0.007 \\
\hline fr_thiophene & Number of thiophene rings & 0.006 \\
\hline Acceptor:Poslonizable-6.00 & $\begin{array}{l}\text { Number of pairs of acceptor-poslonazable atoms } \\
\text { within } 6 \text { bonds }\end{array}$ & 0.006 \\
\hline Donor:Donor-2.00 & $\begin{array}{l}\text { Number of pairs of donor-donor atoms within } 2 \\
\text { bonds }\end{array}$ & 0.006 \\
\hline Aromatic:Poslonizable-5.00 & $\begin{array}{l}\text { Number of pairs of acceptor-poslonazable atoms } \\
\text { within } 5 \text { bonds }\end{array}$ & 0.006 \\
\hline Aromatic:Poslonizable-4.00 & $\begin{array}{l}\text { Number of pairs of acceptor-poslonazable atoms } \\
\text { within } 4 \text { bonds }\end{array}$ & 0.006 \\
\hline fr_unbrch_alkane & $\begin{array}{l}\text { Number of unbranched alkanes of at least } 4 \\
\text { members (excludes halogenated alkanes) }\end{array}$ & 0.005 \\
\hline fr_nitrile & Number of nitriles & 0.005 \\
\hline Acceptor:Neglonizable-1.00 & $\begin{array}{l}\text { Number of pairs of acceptor-neglonazable atoms } \\
\text { within } 1 \text { bond }\end{array}$ & 0.005 \\
\hline fr_nitro_arom & Number of nitro benzene ring substituents & 0.004 \\
\hline Tox_2 & {$[\mathrm{CH}]=[\mathrm{CH}] \mathrm{O}$} & 0.004 \\
\hline fr_nitro & Number of nitro groups & 0.004 \\
\hline
\end{tabular}




\begin{tabular}{|l|l|r|}
\hline Hydrophobe:Poslonizable-3.00 & $\begin{array}{l}\text { Number of pairs of hydrophobe-poslonazable } \\
\text { atoms within 3 bonds }\end{array}$ & 0.004 \\
\hline Donor:Neglonizable-1.00 & $\begin{array}{l}\text { Number of pairs of donor-neglonazable atoms } \\
\text { within 1 bond }\end{array}$ & 0.004 \\
\hline fr_azo & Number of azo groups & 0.004 \\
\hline Donor:Poslonizable-4.00 & $\begin{array}{l}\text { Number of pairs of donor-neglonazable atoms } \\
\text { within 2 bonds }\end{array}$ & 0.004 \\
\hline Poslonizable:Poslonizable-5.00 & $\begin{array}{l}\text { Number of pairs of poslonazable-poslonazable } \\
\text { atoms within 5 bonds }\end{array}$ & 0.004 \\
\hline Acceptor:Poslonizable-3.00 & $\begin{array}{l}\text { Number of pairs of acceptor-poslonazable atoms } \\
\text { within 3 bonds }\end{array}$ & 0.004 \\
\hline Hydrophobe:Neglonizable-1.00 & $\begin{array}{l}\text { Number of pairs of hydrophobe-neglonazable } \\
\text { atoms within 1 bond }\end{array}$ & 0.003 \\
\hline Acceptor:Poslonizable-2.00 & $\begin{array}{l}\text { Number of pairs of acceptor-poslonazable atoms } \\
\text { within 2 bonds }\end{array}$ & 0.003 \\
\hline fr_hdrzone & Number of hydrazone groups & 0.003 \\
\hline Hydrophobe:Neglonizable-3.00 & $\begin{array}{l}\text { Number of pairs of hydrophobe-neglonazable } \\
\text { atoms within 3 bonds }\end{array}$ & 0.002 \\
\hline Acceptor:Poslonizable-1.00 & $\begin{array}{l}\text { Number of pairs of acceptor-poslonazable atoms } \\
\text { within 1 bond }\end{array}$ & 0.002 \\
\hline Aromatic:Neglonizable-1.00 & $\begin{array}{l}\text { Number of pairs of aromatic-neglonazable atoms } \\
\text { within 1 bond }\end{array}$ & 0.002 \\
\hline fr_C_S & Number of thiocarbonyl \\
\hline Donor:Poslonizable-1.00 & $\begin{array}{l}\text { Number of pairs of donor-poslonazable atoms } \\
\text { within 1 bond }\end{array}$ & $\begin{array}{l}\text { Number of pairs of donor-neglonazable atoms } \\
\text { within 2 bonds }\end{array}$ \\
\hline Donor:Neglonizable-2.00 & & 0.002 \\
\hline
\end{tabular}

Table S3. Feature importance for predictive model of PPI inhibitors targeting the CD4/gp120 complex.

\begin{tabular}{|l|l|r|}
\hline Feature & Description & Importance \\
\hline fr_piperzine & Number of piperzine rings & 0.559 \\
\hline PEOE_VSA13 & $\begin{array}{l}\text { MOE-type descriptors, partial charges and } \\
\text { surface area contributions }\end{array}$ & 0.270 \\
\hline fr_amide & Number of amides & 0.169 \\
\hline Poslonizable:Poslonizable-1.00 & $\begin{array}{l}\text { Number of pairs of poslonazable- } \\
\text { poslonazable atoms within 1 bond }\end{array}$ & 0.002 \\
\hline
\end{tabular}

Table S4. Feature importance for predictive model of PPI inhibitors targeting the Cyclophilins complex.

\begin{tabular}{|l|l|r|}
\hline Feature & Description & Importance \\
\hline fr_amide & Number of amides & 0.102 \\
\hline Aromatic:Donor-1.00 & $\begin{array}{l}\text { Number of pairs of aromatic-donor atoms within } \\
1 \text { bond }\end{array}$ & 0.074 \\
\hline fr_Ar_N & Number of aromatic nitrogens & 0.067 \\
\hline Chi1n & Molecular connectivity index & 0.059 \\
\hline SMR_VSA3 & $\begin{array}{l}\text { MOE-type descriptors, molar refractivity and } \\
\text { surface area contributions }\end{array}$ & 0.054 \\
\hline
\end{tabular}




\begin{tabular}{|c|c|c|}
\hline Donor:Donor-5.00 & $\begin{array}{l}\text { Number of pairs of donor-donor atoms within } 5 \\
\text { bonds }\end{array}$ & 0.046 \\
\hline SMR_VSA10 & $\begin{array}{l}\text { MOE-type descriptors, molar refractivity and } \\
\text { surface area contributions }\end{array}$ & 0.044 \\
\hline Donor:Hydrophobe-1.00 & $\begin{array}{l}\text { Number of pairs of donor-hydrophobe atoms } \\
\text { within } 1 \text { bond }\end{array}$ & 0.039 \\
\hline fr_benzene & Number of benzene rings & 0.038 \\
\hline Aromatic:Aromatic-3.00 & $\begin{array}{l}\text { Number of pairs of aromatic-aromatic atoms } \\
\text { within } 3 \text { bonds }\end{array}$ & 0.033 \\
\hline fr_pyridine & Number of pyridine rings & 0.029 \\
\hline Aromatic:Hydrophobe- 1.00 & $\begin{array}{l}\text { Number of pairs of aromatic-hydrophobe atoms } \\
\text { within } 1 \text { bond }\end{array}$ & 0.027 \\
\hline Poslonizable:Poslonizable-2.00 & $\begin{array}{l}\text { Number of pairs of poslonizable-poslonizable } \\
\text { atoms within } 2 \text { bonds }\end{array}$ & 0.027 \\
\hline Aromatic:Aromatic-2.00 & $\begin{array}{l}\text { Number of pairs of aromatic-aromatic atoms } \\
\text { within } 2 \text { bonds }\end{array}$ & 0.027 \\
\hline Aromatic:Hydrophobe-2.00 & $\begin{array}{l}\text { Number of pairs of aromatic-hydrophobe atoms } \\
\text { within } 2 \text { bonds }\end{array}$ & 0.026 \\
\hline SMR_VSA6 & $\begin{array}{l}\text { MOE-type descriptors, molar refractivity and } \\
\text { surface area contributions }\end{array}$ & 0.025 \\
\hline Aromatic:Aromatic-1.00 & $\begin{array}{l}\text { Number of pairs of aromatic-aromatic atoms } \\
\text { within } 1 \text { bond }\end{array}$ & 0.025 \\
\hline Acceptor:Donor-1.00 & $\begin{array}{l}\text { Number of pairs of acceptor-donor atoms within } \\
1 \text { bond }\end{array}$ & 0.023 \\
\hline Poslonizable:Poslonizable-1.00 & $\begin{array}{l}\text { Number of pairs of poslonizable-poslonizable } \\
\text { atoms within } 1 \text { bond }\end{array}$ & 0.021 \\
\hline Acceptor:Acceptor-4.00 & $\begin{array}{l}\text { Number of pairs of acceptor-acceptor atoms } \\
\text { within } 4 \text { bonds }\end{array}$ & 0.021 \\
\hline Hydrophobe:Hydrophobe-2.00 & $\begin{array}{l}\text { Number of pairs of hydrophobe-hydrophobe } \\
\text { atoms within } 2 \text { bonds }\end{array}$ & 0.019 \\
\hline Acceptor:Acceptor-1.00 & $\begin{array}{l}\text { Number of pairs of acceptor-acceptor atoms } \\
\text { within } 1 \text { bond }\end{array}$ & 0.019 \\
\hline Aromatic:Poslonizable-1.00 & $\begin{array}{l}\text { Number of pairs of aromatic-poslonizable atoms } \\
\text { within } 1 \text { bond }\end{array}$ & 0.017 \\
\hline Hydrophobe:Hydrophobe-1.00 & $\begin{array}{l}\text { Number of pairs of hydrophobe-hydrophobe } \\
\text { atoms within } 1 \text { bond }\end{array}$ & 0.017 \\
\hline Acceptor:Acceptor-2.00 & $\begin{array}{l}\text { Number of pairs of acceptor-acceptor atoms } \\
\text { within } 2 \text { bonds }\end{array}$ & 0.016 \\
\hline Acceptor:Hydrophobe-2.00 & $\begin{array}{l}\text { Number of pairs of acceptor-hydrophobe atoms } \\
\text { within } 2 \text { bonds }\end{array}$ & 0.015 \\
\hline Donor:Poslonizable-1.00 & $\begin{array}{l}\text { Number of pairs of donor-poslonizable atoms } \\
\text { within } 1 \text { bond }\end{array}$ & 0.014 \\
\hline Hydrophobe:Neglonizable-2.00 & $\begin{array}{l}\text { Number of pairs of hydrophobe-neglonizable } \\
\text { atoms within } 2 \text { bonds }\end{array}$ & 0.012 \\
\hline Hydrophobe:Neglonizable-1.00 & $\begin{array}{l}\text { Number of pairs of hydrophobe-neglonizable } \\
\text { atoms within } 1 \text { bond }\end{array}$ & 0.011 \\
\hline Donor:Poslonizable-2.00 & $\begin{array}{l}\text { Number of pairs of donor-poslonizable atoms } \\
\text { within } 2 \text { bonds }\end{array}$ & 0.011 \\
\hline Neglonizable:Neglonizable-1.00 & Number of pairs of neglonizable-neglonizable & 0.008 \\
\hline
\end{tabular}




\begin{tabular}{|l|l|r|}
\hline & atoms within 1 bond & 0.006 \\
\hline Acceptor:Neglonizable-1.00 & $\begin{array}{l}\text { Number of pairs of acceptor-neglonizable } \\
\text { atoms within 1 bond }\end{array}$ & 0.006 \\
\hline Donor:Neglonizable-2.00 & $\begin{array}{l}\text { Number of pairs of donor-neglonizable atoms } \\
\text { within 2 bonds }\end{array}$ & 0.005 \\
\hline Hydrophobe:Poslonizable-2.00 & $\begin{array}{l}\text { Number of pairs of hydrophobe-poslonizable } \\
\text { atoms within 2 bonds }\end{array}$ & 0.005 \\
\hline Acceptor:Poslonizable-1.00 & $\begin{array}{l}\text { Number of pairs of acceptor-poslonizable atoms } \\
\text { within 1 bond }\end{array}$ & 0.003 \\
\hline Hydrophobe:Poslonizable-1.00 & $\begin{array}{l}\text { Number of pairs of hydrophobe-poslonizable } \\
\text { atoms within 1 bond }\end{array}$ & 0.003 \\
\hline Donor:Neglonizable-3.00 & $\begin{array}{l}\text { Number of pairs of donor-neglonizable atoms } \\
\text { within 3 bonds }\end{array}$ & 0.002 \\
\hline Donor:Neglonizable-1.00 & $\begin{array}{l}\text { Number of pairs of donor-neglonizable atoms } \\
\text { within 1 bond }\end{array}$ \\
\hline Aromatic:Neglonizable-2.00 & $\begin{array}{l}\text { Number of pairs of aromatic-neglonizable } \\
\text { atoms within 2 bonds }\end{array}$ & 0.002 \\
\hline Aromatic:Neglonizable-1.00 & $\begin{array}{l}\text { Number of pairs of aromatic-neglonizable } \\
\text { atoms within 1 bond }\end{array}$ & \\
\hline
\end{tabular}

Table S5. Feature importance for predictive model of PPI inhibitors targeting the Fkbp1a/Fk506 complex.

\begin{tabular}{|l|l|r|}
\hline Feature & Description & Importance \\
\hline NHOHCount & Number of NHs or OHs & 0.103 \\
\hline Acceptor:Acceptor-3.00 & $\begin{array}{l}\text { Number of pairs of acceptor-acceptor atoms } \\
\text { within 3 bonds }\end{array}$ & 0.093 \\
\hline Poslonizable_Count & Number of poslonizable atoms & 0.092 \\
\hline fr_bicyclic & Number of bicyclic structure & 0.081 \\
\hline Aromatic:Aromatic-2.00 & $\begin{array}{l}\text { Number of pairs of aromatic-aromatic atoms } \\
\text { within 2 bonds }\end{array}$ & 0.072 \\
\hline Aromatic_Count & Number of atoms in aromatic rings & 0.059 \\
\hline fr_methoxy & Number of methoxy groups -OCH3 & 0.059 \\
\hline Aromatic:Aromatic-1.00 & $\begin{array}{l}\text { Number of pairs of aromatic-aromatic atoms } \\
\text { within 1 bond }\end{array}$ & 0.053 \\
\hline fr_NH0 & Number of Tertiary amines & 0.051 \\
\hline Aromatic:Hydrophobe-4.00 & $\begin{array}{l}\text { Number of pairs of aromatic-hydrophobe atoms } \\
\text { within 4 bonds }\end{array}$ & 0.050 \\
\hline Hydrophobe:Hydrophobe-1.00 & $\begin{array}{l}\text { Number of pairs of hydrophobe-hydrophobe } \\
\text { atoms within 1 bond }\end{array}$ & 0.032 \\
\hline Donor:Hydrophobe-5.00 & $\begin{array}{l}\text { Number of pairs of donor-hydrophobe atoms } \\
\text { within 5 bonds }\end{array}$ & 0.029 \\
\hline Hydrophobe:Hydrophobe-2.00 & $\begin{array}{l}\text { Number of pairs of hydrophobe-hydrophobe } \\
\text { atoms within 2 bonds }\end{array}$ & 0.028 \\
\hline Acceptor:Aromatic-1.00 & $\begin{array}{l}\text { Number of pairs of acceptor-aromatic atoms } \\
\text { within 1 bond }\end{array}$ & 0.024 \\
\hline Donor:Donor-3.00 & $\begin{array}{l}\text { Number of pairs of donor-donor atoms within 3 } \\
\text { bonds }\end{array}$ & 0.023 \\
\hline
\end{tabular}




\begin{tabular}{|c|c|c|}
\hline Donor:Hydrophobe-2.00 & $\begin{array}{l}\text { Number of pairs of donor-hydrophobe atoms } \\
\text { within } 2 \text { bonds }\end{array}$ & 0.017 \\
\hline Acceptor:Donor-1.00 & $\begin{array}{l}\text { Number of pairs of acceptor-donor atoms within } \\
1 \text { bond }\end{array}$ & 0.016 \\
\hline Donor:Hydrophobe-1.00 & $\begin{array}{l}\text { Number of pairs of donor-hydrophobe atoms } \\
\text { within } 1 \text { bond }\end{array}$ & 0.013 \\
\hline Donor:Donor-2.00 & $\begin{array}{l}\text { Number of pairs of donor-donor atoms within } 2 \\
\text { bonds }\end{array}$ & 0.012 \\
\hline Hydrophobe:Poslonizable-2.00 & $\begin{array}{l}\text { Number of pairs of hydrophobe-poslonazable } \\
\text { atoms within } 2 \text { bonds }\end{array}$ & 0.010 \\
\hline Aromatic:Poslonizable-2.00 & $\begin{array}{l}\text { Number of pairs of aromatic-poslonazable atoms } \\
\text { within } 2 \text { bonds }\end{array}$ & 0.010 \\
\hline Aromatic:Poslonizable-1.00 & $\begin{array}{l}\text { Number of pairs of aromatic-poslonazable atoms } \\
\text { within } 1 \text { bond }\end{array}$ & 0.008 \\
\hline Neglonizable:Neglonizable-1.00 & $\begin{array}{l}\text { Number of pairs of neglonazable-neglonazable } \\
\text { atoms within } 1 \text { bond }\end{array}$ & 0.007 \\
\hline Neglonizable:Neglonizable-2.00 & $\begin{array}{l}\text { Number of pairs of neglonazable-neglonazable } \\
\text { atoms within } 2 \text { bonds }\end{array}$ & 0.006 \\
\hline Neglonizable:Neglonizable-3.00 & $\begin{array}{l}\text { Number of pairs of neglonazable-neglonazable } \\
\text { atoms within } 3 \text { bonds }\end{array}$ & 0.006 \\
\hline Donor:Donor-1.00 & $\begin{array}{l}\text { Number of pairs of donor-donor atoms within } 1 \\
\text { bond }\end{array}$ & 0.005 \\
\hline fr_oxazole & Number of oxazole rings & 0.005 \\
\hline Neglonizable:Neglonizable-4.00 & $\begin{array}{l}\text { Number of pairs of neglonazable-neglonazable } \\
\text { atoms within } 4 \text { bonds }\end{array}$ & 0.005 \\
\hline Poslonizable:Poslonizable-1.00 & $\begin{array}{l}\text { Number of pairs of poslonazable-poslonazable } \\
\text { atoms within } 1 \text { bond }\end{array}$ & 0.004 \\
\hline fr_sulfone & Number of sulfone groups & 0.004 \\
\hline Acceptor:Neglonizable-1.00 & $\begin{array}{l}\text { Number of pairs of acceptor-neglonazable atoms } \\
\text { within } 1 \text { bond }\end{array}$ & 0.004 \\
\hline Hydrophobe:Poslonizable-1.00 & $\begin{array}{l}\text { Number of pairs of hydrophobe-neglonazable } \\
\text { atoms within } 1 \text { bond }\end{array}$ & 0.003 \\
\hline Poslonizable:Poslonizable-2.00 & $\begin{array}{l}\text { Number of pairs of poslonazable-poslonazable } \\
\text { atoms within } 2 \text { bonds }\end{array}$ & 0.003 \\
\hline Poslonizable:Poslonizable-3.00 & $\begin{array}{l}\text { Number of pairs of poslonazable-poslonazable } \\
\text { atoms within } 3 \text { bonds }\end{array}$ & 0.003 \\
\hline Acceptor:Poslonizable-1.00 & $\begin{array}{l}\text { Number of pairs of acceptor-poslonazable atoms } \\
\text { within } 1 \text { bond }\end{array}$ & 0.002 \\
\hline Hydrophobe:Neglonizable-3.00 & $\begin{array}{l}\text { Number of pairs of hydrophobe-neglonazable } \\
\text { atoms within } 3 \text { bonds }\end{array}$ & 0.002 \\
\hline Aromatic:Neglonizable-3.00 & $\begin{array}{l}\text { Number of pairs of aromatic-neglonazable atoms } \\
\text { within } 3 \text { bonds }\end{array}$ & 0.002 \\
\hline Donor:Poslonizable-1.00 & $\begin{array}{l}\text { Number of pairs of donor-poslonazable atoms } \\
\text { within } 1 \text { bond }\end{array}$ & 0.001 \\
\hline Donor:Poslonizable-2.00 & $\begin{array}{l}\text { Number of pairs of donor-poslonazable atoms } \\
\text { within } 2 \text { bonds }\end{array}$ & 0.001 \\
\hline
\end{tabular}


Table S6. Feature importance for predictive model of PPI inhibitors targeting the HIF1-alpha/p300 complex.

\begin{tabular}{|c|c|c|}
\hline Feature & Descriptor & Importance \\
\hline SMR_VSA3 & $\begin{array}{l}\text { MOE-type descriptors, molar refractivity and } \\
\text { surface area contributions }\end{array}$ & 0.234 \\
\hline SlogP_VSA8 & $\begin{array}{l}\text { MOE-type descriptors, LogP and surface area } \\
\text { contributions }\end{array}$ & 0.127 \\
\hline RingCount & Number of aromatic rings & 0.126 \\
\hline Acceptor:Donor-1.00 & $\begin{array}{l}\text { Number of pairs of acceptor-donor atoms } \\
\text { within } 1 \text { bond }\end{array}$ & 0.100 \\
\hline VSA_EState10 & $\begin{array}{l}\text { MOE-type descriptors, surface area } \\
\text { contributions and EState indices }\end{array}$ & 0.081 \\
\hline Acceptor:Poslonizable-1.00 & $\begin{array}{l}\text { Number of pairs of acceptor-poslonazable } \\
\text { atoms within } 1 \text { bond }\end{array}$ & 0.061 \\
\hline Acceptor:Neglonizable-1.00 & $\begin{array}{l}\text { Number of pairs of acceptor-poslonazable } \\
\text { atoms within } 1 \text { bond }\end{array}$ & 0.049 \\
\hline fr_Al_OH & Number of aliphatic hydroxyl groups & 0.046 \\
\hline Neglonizable:Neglonizable-2.00 & $\begin{array}{l}\text { Number of pairs of neglonazable-neglonazable } \\
\text { atoms within } 2 \text { bonds }\end{array}$ & 0.032 \\
\hline Neglonizable:Neglonizable-1.00 & $\begin{array}{l}\text { Number of pairs of neglonazable-neglonazable } \\
\text { atoms within } 1 \text { bond }\end{array}$ & 0.029 \\
\hline Donor:Poslonizable-6.00 & $\begin{array}{l}\text { Number of pairs of donor-poslonazable atoms } \\
\text { within } 6 \text { bonds }\end{array}$ & 0.027 \\
\hline Aromatic:Poslonizable-1.00 & $\begin{array}{l}\text { Number of pairs of aromatic-poslonazable } \\
\text { atoms within } 1 \text { bond }\end{array}$ & 0.021 \\
\hline Donor:Neglonizable-1.00 & $\begin{array}{l}\text { Number of pairs of donor-neglonazable atoms } \\
\text { within } 1 \text { bond }\end{array}$ & 0.018 \\
\hline Donor:Donor-1.00 & $\begin{array}{l}\text { Number of pairs of donor-donor atoms within } 1 \\
\text { bond }\end{array}$ & 0.016 \\
\hline Hydrophobe:Neglonizable-1.00 & $\begin{array}{l}\text { Number of pairs of hydrophobe-neglonazable } \\
\text { atoms within } 1 \text { bond }\end{array}$ & 0.013 \\
\hline Aromatic:Neglonizable-1.00 & $\begin{array}{l}\text { Number of pairs of aromatic-neglonazable } \\
\text { atoms within } 1 \text { bond }\end{array}$ & 0.010 \\
\hline Hydrophobe:Poslonizable-1.00 & $\begin{array}{l}\text { Number of pairs of hydrophobe-poslonazable } \\
\text { atoms within } 1 \text { bond }\end{array}$ & 0.005 \\
\hline fr_alkyl_carbamate & $\begin{array}{l}\text { Number of alkyl carbamates (subject to } \\
\text { hydrolysis) }\end{array}$ & 0.002 \\
\hline Neglonizable:Poslonizable-1.00 & $\begin{array}{l}\text { Number of pairs of neglonazable-poslonazable } \\
\text { atoms within } 1 \text { bond }\end{array}$ & 0.001 \\
\hline Neglonizable:Poslonizable-2.00 & $\begin{array}{l}\text { Number of pairs of neglonazable-poslonazable } \\
\text { atoms within } 1 \text { bonds }\end{array}$ & 0.001 \\
\hline
\end{tabular}

Table S7. Feature importance for predictive model of PPI inhibitors targeting the Integrins complex.

\begin{tabular}{|l|l|r|}
\hline Feature & Description & Importance \\
\hline fr_Al_COO & Number of aliphatic carboxylic acids & 0.245 \\
\hline PEOE_VSA14 & $\begin{array}{l}\text { MOE-type descriptors, partial charges and } \\
\text { surface area contributions }\end{array}$ & 0.050 \\
\hline
\end{tabular}




\begin{tabular}{|c|c|c|}
\hline Donor:Neglonizable-2.00 & $\begin{array}{l}\text { Number of pairs of donor-neglonazable atoms } \\
\text { within } 2 \text { bonds }\end{array}$ & 0.039 \\
\hline fr_NH1 & Number of Secondary amines & 0.036 \\
\hline PEOE_VSA12 & $\begin{array}{l}\text { MOE-type descriptors, partial charges and } \\
\text { surface area contributions }\end{array}$ & 0.035 \\
\hline Hydrophobe:Neglonizable-4.00 & $\begin{array}{l}\text { Number of pairs of hydrophobe-neglonazable } \\
\text { atoms within } 4 \text { bonds }\end{array}$ & 0.035 \\
\hline Aromatic:Aromatic-1.00 & $\begin{array}{l}\text { Number of pairs of aromatic-aromatic atoms } \\
\text { within } 1 \text { bond }\end{array}$ & 0.033 \\
\hline Hydrophobe:Neglonizable-3.00 & $\begin{array}{l}\text { Number of pairs of hydrophobe-neglonazable } \\
\text { atoms within } 3 \text { bonds }\end{array}$ & 0.031 \\
\hline PEOE_VSA13 & $\begin{array}{l}\text { MOE-type descriptors, partial charges and } \\
\text { surface area contributions }\end{array}$ & 0.031 \\
\hline Hydrophobe:Neglonizable-2.00 & $\begin{array}{l}\text { Number of pairs of hydrophobe-neglonazable } \\
\text { atoms within } 2 \text { bonds }\end{array}$ & 0.029 \\
\hline Aromatic_Count & Number of atoms in aromatic rings & 0.024 \\
\hline SlogP_VSA2 & $\begin{array}{l}\text { MOE-type descriptors, LogP and surface area } \\
\text { contributions }\end{array}$ & 0.024 \\
\hline MolWt & Molecular weight & 0.023 \\
\hline PEOE_VSA2 & $\begin{array}{l}\text { MOE-type descriptors, partial charges and } \\
\text { surface area contributions }\end{array}$ & 0.022 \\
\hline fr_amidine & Number of amidine groups & 0.021 \\
\hline SlogP_VSA5 & $\begin{array}{l}\text { MOE-type descriptors, LogP and surface area } \\
\text { contributions }\end{array}$ & 0.020 \\
\hline Hydrophobe:Hydrophobe-4.00 & $\begin{array}{l}\text { Number of pairs of hydrophobe-hydrophobe } \\
\text { atoms within } 4 \text { bonds }\end{array}$ & 0.018 \\
\hline HallKierAlpha & Hall-Kier alpha value & 0.016 \\
\hline Donor:Donor-2.00 & $\begin{array}{l}\text { Number of pairs of donor-donor atoms within } \\
2 \text { bonds }\end{array}$ & 0.014 \\
\hline Donor:Donor-5.00 & $\begin{array}{l}\text { Number of pairs of donor-donor atoms within } \\
5 \text { bonds }\end{array}$ & 0.014 \\
\hline fr_pyridine & Number of pyridine rings & 0.014 \\
\hline Donor:Donor-6.00 & $\begin{array}{l}\text { Number of pairs of donor-donor atoms within } \\
6 \text { bonds }\end{array}$ & 0.013 \\
\hline Acceptor:Acceptor-1.00 & $\begin{array}{l}\text { Number of pairs of acceptor-acceptor atoms } \\
\text { within } 1 \text { bond }\end{array}$ & 0.013 \\
\hline Acceptor:Aromatic-5.00 & $\begin{array}{l}\text { Number of pairs of acceptor-aromatic atoms } \\
\text { within } 5 \text { bonds }\end{array}$ & 0.013 \\
\hline SlogP_VSA4 & $\begin{array}{l}\text { MOE-type descriptors, LogP and surface area } \\
\text { contributions }\end{array}$ & 0.012 \\
\hline Acceptor:Acceptor-6.00 & $\begin{array}{l}\text { Number of pairs of acceptor-acceptor atoms } \\
\text { within } 6 \text { bonds }\end{array}$ & 0.012 \\
\hline Donor:Donor-4.00 & $\begin{array}{l}\text { Number of pairs of donor-donor atoms within } \\
4 \text { bonds }\end{array}$ & 0.011 \\
\hline Donor:Donor-3.00 & $\begin{array}{l}\text { Number of pairs of donor-donor atoms within } \\
3 \text { bonds }\end{array}$ & 0.011 \\
\hline FCount & Number of fluorine atoms & 0.010 \\
\hline
\end{tabular}




\begin{tabular}{|c|c|c|}
\hline fr_barbitur & Number of barbiturate groups & 0.007 \\
\hline fr_alkyl_halide & Number of alkyl halides & 0.007 \\
\hline fr_Imine & Number of Imines & 0.007 \\
\hline fr_sulfonamd & Number of sulfonamides & 0.007 \\
\hline fr_piperdine & Number of piperdine rings & 0.007 \\
\hline fr_imide & Number of imide groups & 0.007 \\
\hline Aromatic:Poslonizable-6.00 & $\begin{array}{l}\text { Number of pairs of aromatic-poslonazable } \\
\text { atoms within } 6 \text { bonds }\end{array}$ & 0.006 \\
\hline fr_ArN & $\begin{array}{l}\text { Number of } \mathrm{N} \text { functional groups attached to } \\
\text { aromatics }\end{array}$ & 0.006 \\
\hline Donor:Poslonizable-4.00 & $\begin{array}{l}\text { Number of pairs of donor-poslonazable atoms } \\
\text { within } 4 \text { bonds }\end{array}$ & 0.005 \\
\hline fr_Ndealkylation1 & Number of XCCNR groups & 0.005 \\
\hline Donor:Poslonizable-6.00 & $\begin{array}{l}\text { Number of pairs of donor-poslonazable atoms } \\
\text { within } 6 \text { bonds }\end{array}$ & 0.005 \\
\hline Poslonizable:Poslonizable-2.00 & $\begin{array}{l}\text { Number of pairs of poslonazable- } \\
\text { poslonazable atoms within } 2 \text { bonds }\end{array}$ & 0.005 \\
\hline Donor:Poslonizable-5.00 & $\begin{array}{l}\text { Number of pairs of donor-poslonazable atoms } \\
\text { within } 5 \text { bonds }\end{array}$ & 0.005 \\
\hline Donor:Poslonizable-2.00 & $\begin{array}{l}\text { Number of pairs of donor-poslonazable atoms } \\
\text { within } 2 \text { bonds }\end{array}$ & 0.005 \\
\hline Acceptor:Poslonizable-6.00 & $\begin{array}{l}\text { Number of pairs of acceptor-poslonazable } \\
\text { atoms within } 6 \text { bonds }\end{array}$ & 0.005 \\
\hline Poslonizable:Poslonizable-1.00 & $\begin{array}{l}\text { Number of pairs of poslonazable- } \\
\text { poslonazable atoms within } 1 \text { bond }\end{array}$ & 0.005 \\
\hline Hydrophobe:Poslonizable-5.00 & $\begin{array}{l}\text { Number of pairs of hydrophobe-poslonazable } \\
\text { atoms within } 5 \text { bonds }\end{array}$ & 0.005 \\
\hline Aromatic:Poslonizable-2.00 & $\begin{array}{l}\text { Number of pairs of aromatic-poslonazable } \\
\text { atoms within } 2 \text { bonds }\end{array}$ & 0.004 \\
\hline Poslonizable:Poslonizable-3.00 & $\begin{array}{l}\text { Number of pairs of poslonazble-poslonazable } \\
\text { atoms within } 3 \text { bonds }\end{array}$ & 0.004 \\
\hline Acceptor:Poslonizable-4.00 & $\begin{array}{l}\text { Number of pairs of acceptor-poslonazable } \\
\text { atoms within } 4 \text { bonds }\end{array}$ & 0.004 \\
\hline Poslonizable:Poslonizable-5.00 & $\begin{array}{l}\text { Number of pairs of poslonazble-poslonazable } \\
\text { atoms within } 5 \text { bonds }\end{array}$ & 0.004 \\
\hline Poslonizable:Poslonizable-6.00 & $\begin{array}{l}\text { Number of pairs of poslonazble-poslonazable } \\
\text { atoms within } 6 \text { bonds }\end{array}$ & 0.004 \\
\hline Aromatic:Poslonizable-1.00 & $\begin{array}{l}\text { Number of pairs of aromatic-poslonazable } \\
\text { atoms within } 1 \text { bond }\end{array}$ & 0.003 \\
\hline Acceptor:Poslonizable-1.00 & $\begin{array}{l}\text { Number of pairs of acceptor-poslonazable } \\
\text { atoms within } 1 \text { bond }\end{array}$ & 0.002 \\
\hline Hydrophobe:Poslonizable-1.00 & $\begin{array}{l}\text { Number of pairs of hydrophobe-poslonazable } \\
\text { atoms within } 1 \text { bond }\end{array}$ & 0.002 \\
\hline fr_nitrile & Number of nitriles & 0.002 \\
\hline Tox_31 & 1 a a a a2 a 1 a3 a( a 2) a a a a 3 & 0.001 \\
\hline fr_aldehyde & Number of aldehydes & 0.001 \\
\hline Neglonizable:Poslonizable-2.00 & Number of pairs of neglonazable- & 0.001 \\
\hline
\end{tabular}




\begin{tabular}{|l|l|r|}
\hline & poslonazable atoms within 2 bonds & 0.001 \\
\hline fr_SH & Number of thiol groups & 0.001 \\
\hline fr_lactone & Number of cyclic esters (lactones) & 0.001 \\
\hline Neglonizable:Poslonizable-6.00 & $\begin{array}{l}\text { Number of pairs of neglonazable- } \\
\text { poslonazable atoms within 6 bonds }\end{array}$ & \\
\hline
\end{tabular}

Table S8. Feature importance for predictive model of PPI inhibitors targeting the Ledgf/IN complex.

\begin{tabular}{|c|c|c|}
\hline Feature & Description & Importance \\
\hline Acceptor:Aromatic-4.00 & $\begin{array}{l}\text { Number of pairs of acceptor-aromatic atoms } \\
\text { within } 4 \text { bonds }\end{array}$ & 0.059 \\
\hline SMR_VSA3 & $\begin{array}{l}\text { MOE-type descriptors, molar refractivity and } \\
\text { surface area contributions }\end{array}$ & 0.052 \\
\hline fr_C_O_noCOO & Number of carbonyl O, excluding $\mathrm{COOH}$ & 0.045 \\
\hline fr_amide & Number of amides & 0.038 \\
\hline Acceptor:Hydrophobe-2.00 & $\begin{array}{l}\text { Number of pairs of acceptor-hydrophobe atoms } \\
\text { within } 2 \text { bonds }\end{array}$ & 0.037 \\
\hline SlogP_VSA8 & $\begin{array}{l}\text { MOE-type descriptors, LogP and surface area } \\
\text { contributions }\end{array}$ & 0.035 \\
\hline Acceptor:Hydrophobe-4.00 & $\begin{array}{l}\text { Number of pairs of acceptor-hydrophobe atoms } \\
\text { within } 4 \text { bonds }\end{array}$ & 0.035 \\
\hline fr_pyridine & Number of pyridine rings & 0.032 \\
\hline Acceptor:Acceptor-5.00 & $\begin{array}{l}\text { Number of pairs of acceptor-acceptor atoms } \\
\text { within } 5 \text { bonds }\end{array}$ & 0.029 \\
\hline PEOE_VSA2 & $\begin{array}{l}\text { MOE-type descriptors, partial charges and } \\
\text { surface area contributions }\end{array}$ & 0.024 \\
\hline Acceptor:Hydrophobe-5.00 & $\begin{array}{l}\text { Number of pairs of acceptor-hydrophobe atoms } \\
\text { within } 5 \text { bonds }\end{array}$ & 0.024 \\
\hline HallKierAlpha & Hall-Kier alpha value & 0.022 \\
\hline Hydrophobe:Hydrophobe-3.00 & $\begin{array}{l}\text { Number of pairs of hydrophobe-hydrophobe } \\
\text { atoms within } 3 \text { bonds }\end{array}$ & 0.022 \\
\hline PEOE_VSA10 & $\begin{array}{l}\text { MOE-type descriptors, partial charges and } \\
\text { surface area contributions }\end{array}$ & 0.022 \\
\hline Chi2n & Molecular connectivity index & 0.021 \\
\hline Donor:Neglonizable-4.00 & $\begin{array}{l}\text { Number of pairs of donor-neglonazable atoms } \\
\text { within } 4 \text { bonds }\end{array}$ & 0.021 \\
\hline SlogP_VSA2 & $\begin{array}{l}\text { MOE-type descriptors, LogP and surface area } \\
\text { contributions }\end{array}$ & 0.020 \\
\hline SMR_VSA2 & $\begin{array}{l}\text { MOE-type descriptors, molar refractivity and } \\
\text { surface area contributions }\end{array}$ & 0.019 \\
\hline SlogP_VSA4 & $\begin{array}{l}\text { MOE-type descriptors, LogP and surface area } \\
\text { contributions }\end{array}$ & 0.019 \\
\hline ChiOv & Molecular connectivity index & 0.019 \\
\hline PEOE_VSA6 & $\begin{array}{l}\text { MOE-type descriptors, partial charges and } \\
\text { surface area contributions }\end{array}$ & 0.019 \\
\hline Chio & Molecular connectivity index & 0.018 \\
\hline Kappa1 & Molecular shape index & 0.018 \\
\hline
\end{tabular}




\begin{tabular}{|c|c|c|}
\hline Aromatic:Hydrophobe-4.00 & $\begin{array}{l}\text { Number of pairs of aromatic-hydrophobe atoms } \\
\text { within } 4 \text { bonds }\end{array}$ & 0.017 \\
\hline Chi1v & Molecular connectivity index & 0.017 \\
\hline PEOE_VSA7 & $\begin{array}{l}\text { MOE-type descriptors, partial charges and } \\
\text { surface area contributions }\end{array}$ & 0.015 \\
\hline Donor:Hydrophobe-5.00 & $\begin{array}{l}\text { Number of pairs of donor-hydrophobe atoms } \\
\text { within } 5 \text { bonds }\end{array}$ & 0.015 \\
\hline PEOE_VSA12 & $\begin{array}{l}\text { MOE-type descriptors, partial charges and } \\
\text { surface area contributions }\end{array}$ & 0.015 \\
\hline Aromatic:Donor-3.00 & $\begin{array}{l}\text { Number of pairs of aromatic-donor atoms } \\
\text { within } 3 \text { bonds }\end{array}$ & 0.015 \\
\hline fr_nitrile & Number of nitriles & 0.013 \\
\hline Acceptor:Donor-5.00 & $\begin{array}{l}\text { Number of pairs of acceptor-donor atoms } \\
\text { within } 5 \text { bonds }\end{array}$ & 0.013 \\
\hline Acceptor:Donor-2.00 & $\begin{array}{l}\text { Number of pairs of acceptor-donor atoms } \\
\text { within } 2 \text { bonds }\end{array}$ & 0.013 \\
\hline Donor:Donor-3.00 & $\begin{array}{l}\text { Number of pairs of donor-donor atoms within } 3 \\
\text { bonds }\end{array}$ & 0.012 \\
\hline fr_Ndealkylation2 & $\begin{array}{l}\text { Number of tert-alicyclic amines (no } \\
\text { heteroatoms, not quinine-like bridged N) }\end{array}$ & 0.011 \\
\hline Donor:Donor-5.00 & $\begin{array}{l}\text { Number of pairs of donor-donor atoms within } 5 \\
\text { bonds }\end{array}$ & 0.011 \\
\hline Donor:Donor-6.00 & $\begin{array}{l}\text { Number of pairs of donor-donor atoms within } 6 \\
\text { bonds }\end{array}$ & 0.011 \\
\hline Donor:Donor-2.00 & $\begin{array}{l}\text { Number of pairs of donor-donor atoms within } 2 \\
\text { bonds }\end{array}$ & 0.010 \\
\hline fr_C_S & Number of thiocarbonyl & 0.009 \\
\hline fr_hdrzone & Number of hydrazone groups & 0.009 \\
\hline fr_urea & Number of urea groups & 0.009 \\
\hline fr_quatN & Number of quarternary nitrogens & 0.008 \\
\hline Donor:Donor-1.00 & $\begin{array}{l}\text { Number of pairs of donor-donor atoms within } 1 \\
\text { bond }\end{array}$ & 0.008 \\
\hline PEOE_VSA5 & $\begin{array}{l}\text { MOE-type descriptors, partial charges and } \\
\text { surface area contributions }\end{array}$ & 0.008 \\
\hline Aromatic:Poslonizable-1.00 & $\begin{array}{l}\text { Number of pairs of aromatic-poslonazable } \\
\text { atoms within } 1 \text { bond }\end{array}$ & 0.008 \\
\hline SlogP_VSA7 & $\begin{array}{l}\text { MOE-type descriptors, LogP and surface area } \\
\text { contributions }\end{array}$ & 0.008 \\
\hline Poslonizable:Poslonizable-2.00 & $\begin{array}{l}\text { Number of pairs of poslonazable-poslonazable } \\
\text { atoms within } 2 \text { bonds }\end{array}$ & 0.007 \\
\hline Donor:Poslonizable-1.00 & $\begin{array}{l}\text { Number of pairs of donor-poslonazable atoms } \\
\text { within } 1 \text { bond }\end{array}$ & 0.006 \\
\hline Poslonizable:Poslonizable-1.00 & $\begin{array}{l}\text { Number of pairs of poslonazable-poslonazable } \\
\text { atoms within } 1 \text { bond }\end{array}$ & 0.006 \\
\hline Poslonizable:Poslonizable-3.00 & $\begin{array}{l}\text { Number of pairs of poslonazable-poslonazable } \\
\text { atoms within } 3 \text { bonds }\end{array}$ & 0.006 \\
\hline fr_ArN & $\begin{array}{l}\text { Number of } \mathrm{N} \text { functional groups attached to } \\
\text { aromatics }\end{array}$ & 0.005 \\
\hline
\end{tabular}




\begin{tabular}{|l|l|l|}
\hline fr_unbrch_alkane & $\begin{array}{l}\text { Number of unbranched alkanes of at least 4 } \\
\text { members (excludes halogenated alkanes) }\end{array}$ & 0.005 \\
\hline fr_priamide & Number of primary amides & 0.005 \\
\hline fr_alkyl_halide & Number of alkyl halides & 0.005 \\
\hline fr_nitro_arom & Number of nitro benzene ring substituents & 0.005 \\
\hline fr_nitro & Number of nitro groups & 0.005 \\
\hline fr_ketone & Number of ketones & 0.005 \\
\hline Tox_1 & $\mathrm{O}=\mathrm{N}(\sim \mathrm{O}) \mathrm{a}$ & 0.005 \\
\hline Tox_2 & a[NH2] & 0.004 \\
\hline fr_Imine & Number of Imines & 0.004 \\
\hline fr_Al_OH_noTert & $\begin{array}{l}\text { Number of aliphatic hydroxyl groups excluding } \\
\text { tert-OH }\end{array}$ & 0.003 \\
\hline Donor:Neglonizable-3.00 & $\begin{array}{l}\text { Number of pairs of donor-neglonazable atoms } \\
\text { within 3 bonds }\end{array}$ & 0.003 \\
\hline Tox_12 & {$[\mathrm{OH}, \mathrm{NH} 2][\mathrm{N}, \mathrm{O}]$} & 0.002 \\
\hline fr_azo & Number of azo groups & 0.002 \\
\hline fr_Al_OH & Number of aliphatic hydroxyl groups & 0.002 \\
\hline fr_oxime & Number of oxime groups & 0.002 \\
\hline fr_thiazole & Number of thiazole rings & 0.002 \\
\hline fr_nitro_arom_nonortho & $\begin{array}{l}\text { Number of non-ortho nitro benzene ring } \\
\text { substituents }\end{array}$ & 0.001 \\
\hline fr_Ndealkylation1 & Number of XCCNR groups & 0.001 \\
\hline
\end{tabular}

Table S9. Feature importance for predictive model of PPI inhibitors targeting the Lfa/lcam complex.

\begin{tabular}{|l|l|r|}
\hline Feature & Description & Importance \\
\hline VSA_EState8 & $\begin{array}{l}\text { MOE-type descriptors, surface area } \\
\text { contributions and EState indices }\end{array}$ & 0.132 \\
\hline SlogP_VSA10 & $\begin{array}{l}\text { MOE-type descriptors, LogP and surface } \\
\text { area contributions }\end{array}$ & 0.121 \\
\hline fr_amide & Number of amides & 0.115 \\
\hline SMR_VSA5 & $\begin{array}{l}\text { MOE-type descriptors, molar refractivity and } \\
\text { surface area contributions }\end{array}$ & 0.089 \\
\hline Kappa2 & Molecular shape index & 0.081 \\
\hline Hydrophobe:Hydrophobe-1.00 & $\begin{array}{l}\text { Number of pairs of hydrophobe-hydrophobe } \\
\text { atoms within 1 bond }\end{array}$ & 0.074 \\
\hline fr_bicyclic & Number of bicyclic structures & 0.073 \\
\hline SlogP_VSA5 & $\begin{array}{l}\text { MOE-type descriptors, LogP and surface } \\
\text { area contributions }\end{array}$ & 0.072 \\
\hline fr_imide & Number of imide groups & 0.065 \\
\hline Acceptor:Donor-4.00 & $\begin{array}{l}\text { Number of pairs of acceptor-donor atoms } \\
\text { within 4 bonds }\end{array}$ & 0.063 \\
\hline fr_para_hydroxylation & Number of para-hydroxylation sites & 0.045 \\
\hline Donor:Donor-1.00 & $\begin{array}{l}\text { Number of pairs of donor-donor atoms } \\
\text { within 1 bond }\end{array}$ & 0.034 \\
\hline fr_urea & Number of urea groups & 0.026 \\
\hline
\end{tabular}




\begin{tabular}{|l|l|r|}
\hline fr_guanido & Number of guanidine groups & 0.009 \\
\hline fr_term_acetylene & Number of terminal acetylenes & 0.002 \\
\hline
\end{tabular}

Table S10. Feature importance for predictive model of PPI inhibitors targeting the Mdm2-like/P53 complex.

\begin{tabular}{|c|c|c|}
\hline Feature & Description & Importance \\
\hline VSA_EState10 & $\begin{array}{l}\text { MOE-type descriptors, surface area contributions } \\
\text { and EState indices }\end{array}$ & 0.085 \\
\hline $\mathrm{fr}$ fhalogen & Number of halogens & 0.051 \\
\hline SMR_VSA7 & $\begin{array}{l}\text { MOE-type descriptors, molar refractivity and } \\
\text { surface area contributions }\end{array}$ & 0.045 \\
\hline Donor:Hydrophobe-4.00 & $\begin{array}{l}\text { Number of pairs of donor-hydrophobe atoms } \\
\text { within } 4 \text { bonds }\end{array}$ & 0.040 \\
\hline Aromatic:Aromatic-6.00 & $\begin{array}{l}\text { Number of pairs of aromatic-aromatic atoms } \\
\text { within } 6 \text { bonds }\end{array}$ & 0.031 \\
\hline Aromatic:Aromatic-5.00 & $\begin{array}{l}\text { Number of pairs of aromatic-aromatic atoms } \\
\text { within } 6 \text { bonds }\end{array}$ & 0.026 \\
\hline Chi4n & Molecular connectivity index & 0.025 \\
\hline SMR_VSA9 & $\begin{array}{l}\text { MOE-type descriptors, molar refractivity and } \\
\text { surface area contributions }\end{array}$ & 0.023 \\
\hline Aromatic_Count & Number of atoms in aromatic rings & 0.022 \\
\hline Aromatic:Aromatic-4.00 & $\begin{array}{l}\text { Number of pairs of aromatic-aromatic atoms } \\
\text { within } 4 \text { bonds }\end{array}$ & 0.021 \\
\hline SlogP_VSA8 & $\begin{array}{l}\text { MOE-type descriptors, LogP and surface area } \\
\text { contributions }\end{array}$ & 0.020 \\
\hline fr_aniline & Number of anilines & 0.020 \\
\hline PEOE_VSA8 & $\begin{array}{l}\text { MOE-type descriptors, partial charges and } \\
\text { surface area contributions }\end{array}$ & 0.018 \\
\hline Aromatic:Aromatic-2.00 & $\begin{array}{l}\text { Number of pairs of aromatic-aromatic atoms } \\
\text { within } 2 \text { bonds }\end{array}$ & 0.018 \\
\hline Chi3n & Molecular connectivity index & 0.018 \\
\hline MolWt & Molecular weight & 0.017 \\
\hline fr_NH1 & Number of Secondary amines & 0.017 \\
\hline BalabanJ & Balaban's connectivity topological index & 0.017 \\
\hline PEOE_VSA13 & $\begin{array}{l}\text { MOE-type descriptors, partial charges and } \\
\text { surface area contributions }\end{array}$ & 0.017 \\
\hline fr_bicyclic & Number of bicyclic structures & 0.016 \\
\hline Aromatic:Aromatic-1.00 & $\begin{array}{l}\text { Number of pairs of aromatic-aromatic atoms } \\
\text { within } 1 \text { bond }\end{array}$ & 0.015 \\
\hline Acceptor:Aromatic-1.00 & $\begin{array}{l}\text { Number of pairs of acceptor-aromatic atoms } \\
\text { within } 1 \text { bond }\end{array}$ & 0.015 \\
\hline ChiOn & Molecular connectivity index & 0.014 \\
\hline HeavyAtomCount & Number of heavy atoms & 0.014 \\
\hline fr_Nhpyrrole & Number of H-pyrrole nitrogens & 0.014 \\
\hline fr_aryl_methyl & Number of aryl methyl sites for hydroxylation & 0.013 \\
\hline
\end{tabular}




\begin{tabular}{|c|c|c|}
\hline fr_C_O_noCOO & Number of carbonyl O, excluding $\mathrm{COOH}$ & 0.012 \\
\hline Acceptor:Hydrophobe-2.00 & $\begin{array}{l}\text { Number of pairs of acceptor-hydrophobe atoms } \\
\text { within } 2 \text { bonds }\end{array}$ & 0.012 \\
\hline Acceptor:Acceptor-3.00 & $\begin{array}{l}\text { Number of pairs of acceptor-acceptor atoms } \\
\text { within } 3 \text { bonds }\end{array}$ & 0.012 \\
\hline Hydrophobe:Poslonizable-4.00 & $\begin{array}{l}\text { Number of pairs of hydrophobe-poslonazable } \\
\text { atoms within } 4 \text { bonds }\end{array}$ & 0.012 \\
\hline Acceptor:Aromatic-3.00 & $\begin{array}{l}\text { Number of pairs of acceptor-aromatic atoms } \\
\text { within } 3 \text { bonds }\end{array}$ & 0.012 \\
\hline Acceptor:Donor-1.00 & $\begin{array}{l}\text { Number of pairs of acceptor-donor atoms within } 1 \\
\text { bond }\end{array}$ & 0.011 \\
\hline SMR_VSA1 & $\begin{array}{l}\text { MOE-type descriptors, molar refractivity and } \\
\text { surface area contributions }\end{array}$ & 0.011 \\
\hline Acceptor:Hydrophobe- 6.00 & $\begin{array}{l}\text { Number of pairs of acceptor-hydrophobe atoms } \\
\text { within } 6 \text { bonds }\end{array}$ & 0.011 \\
\hline VSA_EState8 & $\begin{array}{l}\text { MOE-type descriptors surface area contributions } \\
\text { and EState indices }\end{array}$ & 0.011 \\
\hline PEOE_VSA14 & $\begin{array}{l}\text { MOE-type descriptors, partial charges and } \\
\text { surface area contributions }\end{array}$ & 0.011 \\
\hline fr_C_O & Number of carbonyl $\mathrm{O}$ & 0.011 \\
\hline Hydrophobe:Poslonizable-3.00 & $\begin{array}{l}\text { Number of pairs of hydrophobe-poslonazable } \\
\text { atoms within } 3 \text { bonds }\end{array}$ & 0.011 \\
\hline Kappa1 & Molecular shape index & 0.010 \\
\hline fr_pyridine & Number of pyridine rings & 0.010 \\
\hline Hydrophobe:Poslonizable-2.00 & $\begin{array}{l}\text { Number of pairs of hydrophobe-poslonazable } \\
\text { atoms within } 2 \text { bonds }\end{array}$ & 0.010 \\
\hline PEOE_VSA11 & $\begin{array}{l}\text { MOE-type descriptors, partial charges and } \\
\text { surface area contributions }\end{array}$ & 0.010 \\
\hline Acceptor:Hydrophobe-4.00 & $\begin{array}{l}\text { Number of pairs of acceptor-poslonazable atoms } \\
\text { within } 4 \text { bonds }\end{array}$ & 0.009 \\
\hline fr_morpholine & Number of morpholine rings & 0.009 \\
\hline Acceptor:Poslonizable-1.00 & $\begin{array}{l}\text { Number of pairs of acceptor-poslonazable atoms } \\
\text { within } 1 \text { bond }\end{array}$ & 0.009 \\
\hline VSA_EState9 & $\begin{array}{l}\text { MOE-type descriptors surface area contributions } \\
\text { and EState indices }\end{array}$ & 0.009 \\
\hline Donor_Count & Number of hydrogen donors & 0.008 \\
\hline PEOE_VSA9 & $\begin{array}{l}\text { MOE-type descriptors, partial charges and } \\
\text { surface area contributions }\end{array}$ & 0.008 \\
\hline Aromatic:Poslonizable-3.00 & $\begin{array}{l}\text { Number of pairs of aromatic-poslonazable atoms } \\
\text { within } 3 \text { bonds }\end{array}$ & 0.008 \\
\hline Donor:Donor-6.00 & $\begin{array}{l}\text { Number of pairs of donor-donor atoms within } 6 \\
\text { bonds }\end{array}$ & 0.008 \\
\hline fr_piperzine & Number of piperzine rings & 0.007 \\
\hline Donor:Donor-4.00 & $\begin{array}{l}\text { Number of pairs of donor-donor atoms within } 4 \\
\text { bonds }\end{array}$ & 0.007 \\
\hline Acceptor:Poslonizable-5.00 & $\begin{array}{l}\text { Number of pairs of acceptor-poslonazable atoms } \\
\text { within } 5 \text { bonds }\end{array}$ & 0.007 \\
\hline Donor:Donor-3.00 & Number of pairs of donor-donor atoms within 3 & 0.006 \\
\hline
\end{tabular}




\begin{tabular}{|c|c|c|}
\hline & bonds & \\
\hline Hydrophobe:Poslonizable-1.00 & $\begin{array}{l}\text { Number of pairs of hydrophobe-poslonazable } \\
\text { atoms within } 1 \text { bond }\end{array}$ & 0.006 \\
\hline Poslonizable:Poslonizable-3.00 & $\begin{array}{l}\text { Number of pairs of poslonazable-poslonazable } \\
\text { atoms within } 3 \text { bonds }\end{array}$ & 0.006 \\
\hline Neglonizable:Neglonizable-2.00 & $\begin{array}{l}\text { Number of pairs of neglonazable-neglonazable } \\
\text { atoms within } 2 \text { bonds }\end{array}$ & 0.005 \\
\hline fr_Ndealkylation1 & Number of XCCNR groups & 0.005 \\
\hline Donor:Donor-2.00 & $\begin{array}{l}\text { Number of pairs of donor-donor atoms within } 2 \\
\text { bonds }\end{array}$ & 0.005 \\
\hline fr_phenol & Number of phenols & 0.005 \\
\hline FCount & Number of fluorine atoms & 0.005 \\
\hline Donor:Neglonizable-1.00 & $\begin{array}{l}\text { Number of pairs of donor-neglonazable atoms } \\
\text { within } 1 \text { bond }\end{array}$ & 0.004 \\
\hline Neglonizable:Neglonizable-6.00 & $\begin{array}{l}\text { Number of pairs of neglonazable-neglonazable } \\
\text { atoms within } 6 \text { bonds }\end{array}$ & 0.004 \\
\hline Donor:Poslonizable-5.00 & $\begin{array}{l}\text { Number of pairs of donor-neglonazable atoms } \\
\text { within } 5 \text { bonds }\end{array}$ & 0.004 \\
\hline Neglonizable:Neglonizable-3.00 & $\begin{array}{l}\text { Number of pairs of neglonazable-neglonazable } \\
\text { atoms within } 3 \text { bonds }\end{array}$ & 0.004 \\
\hline fr_nitrile & Number of nitriles & 0.004 \\
\hline fr_NH2 & Number of Primary amines & 0.003 \\
\hline Donor:Poslonizable-3.00 & $\begin{array}{l}\text { Number of pairs of donor-poslonazable atoms } \\
\text { within } 3 \text { bonds }\end{array}$ & 0.003 \\
\hline Acceptor:Neglonizable-1.00 & $\begin{array}{l}\text { Number of pairs of acceptor-neglonazable atoms } \\
\text { within } 1 \text { bond }\end{array}$ & 0.003 \\
\hline Neglonizable:Neglonizable-1.00 & $\begin{array}{l}\text { Number of pairs of neglonazable-neglonazable } \\
\text { atoms within } 1 \text { bond }\end{array}$ & 0.003 \\
\hline Tox_1 & $\mathrm{O}=\mathrm{N}(\sim \mathrm{O}) \mathrm{a}$ & 0.003 \\
\hline Donor:Neglonizable-2.00 & $\begin{array}{l}\text { Number of pairs of donor-neglonazable atoms } \\
\text { within } 2 \text { bonds }\end{array}$ & 0.003 \\
\hline fr_imide & Number of imide groups & 0.002 \\
\hline fr_nitro & Number of nitro groups & 0.002 \\
\hline Hydrophobe:Neglonizable-1.00 & $\begin{array}{l}\text { Number of pairs of hydrophobe-neglonazable } \\
\text { atoms within } 1 \text { bond }\end{array}$ & 0.002 \\
\hline Hydrophobe:Neglonizable-3.00 & $\begin{array}{l}\text { Number of pairs of hydrophobe-neglonazable } \\
\text { atoms within } 3 \text { bonds }\end{array}$ & 0.002 \\
\hline Aromatic:Neglonizable-2.00 & $\begin{array}{l}\text { Number of pairs of aromatic-neglonazable atoms } \\
\text { within } 2 \text { bonds }\end{array}$ & 0.002 \\
\hline fr_nitro_arom & Number of nitro benzene ring substituents & 0.002 \\
\hline Aromatic:Neglonizable-4.00 & $\begin{array}{l}\text { Number of pairs of aromatic-neglonazable atoms } \\
\text { within } 4 \text { bonds }\end{array}$ & 0.002 \\
\hline Donor:Poslonizable-1.00 & $\begin{array}{l}\text { Number of pairs of donor-poslonazable atoms } \\
\text { within } 1 \text { bond }\end{array}$ & 0.002 \\
\hline Aromatic:Neglonizable-3.00 & $\begin{array}{l}\text { Number of pairs of aromatic-neglonazable atoms } \\
\text { within } 3 \text { bonds }\end{array}$ & 0.002 \\
\hline Aromatic:Neglonizable-6.00 & Number of pairs of aromatic-neglonazable atoms & 0.002 \\
\hline
\end{tabular}




\begin{tabular}{|l|l|r|}
\hline & within 6 bonds & 0.001 \\
\hline fr_thiophene & Number of thiophene rings & 0.001 \\
\hline fr_HOCCN & $\begin{array}{l}\text { Number of } \mathrm{C}(\mathrm{OH}) \mathrm{CCN} \text {-Ctert-alkyl or } \\
\mathrm{C}(\mathrm{OH}) \mathrm{CCN} \text { Cyclic }\end{array}$ & 0.001 \\
\hline fr_tetrazole & Number of tetrazole rings & 0.001 \\
\hline Tox_33 & a1 a a a a2 a 1 a a3 a( a 2) a a a a 3 & 0.001 \\
\hline fr_ArN & $\begin{array}{l}\text { Number of } \mathrm{N} \text { functional groups attached to } \\
\text { aromatics }\end{array}$ & \\
\hline
\end{tabular}

Table S11. Feature importance for predictive model of PPI inhibitors targeting the Ras/SOS1 complex.

\begin{tabular}{|c|c|c|}
\hline Feature & Description & Importance \\
\hline fr_aryl_methyl & Number of aryl methyl sites for hydroxylation & 0.080 \\
\hline Acceptor:Hydrophobe-1.00 & $\begin{array}{l}\text { Number of pairs of acceptor-hydrophobe } \\
\text { atoms within } 1 \text { bond }\end{array}$ & 0.075 \\
\hline Acceptor:Hydrophobe-6.00 & $\begin{array}{l}\text { Number of pairs of acceptor-hydrophobe } \\
\text { atoms within } 6 \text { bonds }\end{array}$ & 0.051 \\
\hline Aromatic:Hydrophobe-1.00 & $\begin{array}{l}\text { Number of pairs of aromatic-hydrophobe } \\
\text { atoms within } 1 \text { bond }\end{array}$ & 0.047 \\
\hline Hydrophobe:Hydrophobe-1.00 & $\begin{array}{l}\text { Number of pairs of hydrophobe-hydrophobe } \\
\text { atoms within } 1 \text { bond }\end{array}$ & 0.046 \\
\hline Aromatic_Count & Number of atoms in aromatic rings & 0.042 \\
\hline SMR_VSA7 & $\begin{array}{l}\text { MOE-type descriptors, molar refractivity and } \\
\text { surface area contributions }\end{array}$ & 0.041 \\
\hline Hydrophobe:Hydrophobe-4.00 & $\begin{array}{l}\text { Number of pairs of hydrophobe-hydrophobe } \\
\text { atoms within } 4 \text { bonds }\end{array}$ & 0.037 \\
\hline Acceptor:Donor-1.00 & $\begin{array}{l}\text { Number of pairs of acceptor-donor atoms } \\
\text { within } 1 \text { bond }\end{array}$ & 0.037 \\
\hline Donor_Count & Number of hydrogen donors & 0.035 \\
\hline Aromatic:Aromatic-1.00 & $\begin{array}{l}\text { Number of pairs of aromatic-aromatic atoms } \\
\text { within } 1 \text { bond }\end{array}$ & 0.034 \\
\hline Aromatic:Aromatic-5.00 & $\begin{array}{l}\text { Number of pairs of aromatic-aromatic atoms } \\
\text { within } 5 \text { bonds }\end{array}$ & 0.031 \\
\hline Aromatic:Aromatic-3.00 & $\begin{array}{l}\text { Number of pairs of aromatic-aromatic atoms } \\
\text { within } 3 \text { bonds }\end{array}$ & 0.030 \\
\hline fr_piperzine & Number of piperzine rings & 0.030 \\
\hline Aromatic:Aromatic-2.00 & $\begin{array}{l}\text { Number of pairs of aromatic-aromatic atoms } \\
\text { within } 2 \text { bonds }\end{array}$ & 0.029 \\
\hline Chi4n & Molecular connectivity index & 0.029 \\
\hline Aromatic:Hydrophobe-5.00 & $\begin{array}{l}\text { Number of pairs of aromatic-hydrophobe } \\
\text { atoms within } 5 \text { bonds }\end{array}$ & 0.027 \\
\hline Aromatic:Aromatic-4.00 & $\begin{array}{l}\text { Number of pairs of aromatic-aromatic atoms } \\
\text { within } 4 \text { bonds }\end{array}$ & 0.026 \\
\hline fr_sulfide & Number of thioether & 0.018 \\
\hline Neglonizable_Count & Number of neglonizable atoms & 0.016 \\
\hline Donor:Poslonizable-5.00 & $\begin{array}{l}\text { Number of pairs of donor-poslonazable atoms } \\
\text { within } 5 \text { bonds }\end{array}$ & 0.016 \\
\hline
\end{tabular}




\begin{tabular}{|c|c|c|}
\hline fr_quatN & Number of quarternary nitrogens & 0.014 \\
\hline Aromatic:Donor-1.00 & $\begin{array}{l}\text { Number of pairs of aromatic-donor atoms } \\
\text { within } 1 \text { bond }\end{array}$ & 0.014 \\
\hline fr_nitrile & Number of nitriles & 0.013 \\
\hline Donor:Donor-1.00 & $\begin{array}{l}\text { Number of pairs of donor-donor atoms within } 1 \\
\text { bond }\end{array}$ & 0.010 \\
\hline Neglonizable:Neglonizable-1.00 & $\begin{array}{l}\text { Number of pairs of neglonazable- } \\
\text { neglonazable atoms within } 1 \text { bond }\end{array}$ & 0.010 \\
\hline Acceptor:Poslonizable-3.00 & $\begin{array}{l}\text { Number of pairs of acceptor-neglonazable } \\
\text { atoms within } 3 \text { bonds }\end{array}$ & 0.010 \\
\hline Acceptor:Neglonizable-1.00 & $\begin{array}{l}\text { Number of pairs of acceptor-neglonazable } \\
\text { atoms within } 1 \text { bond }\end{array}$ & 0.009 \\
\hline Aromatic:Poslonizable-2.00 & $\begin{array}{l}\text { Number of pairs of aromatic-poslonazable } \\
\text { atoms within } 2 \text { bonds }\end{array}$ & 0.009 \\
\hline Hydrophobe:Poslonizable-1.00 & $\begin{array}{l}\text { Number of pairs of hydrophobe-poslonazable } \\
\text { atoms within } 1 \text { bond }\end{array}$ & 0.009 \\
\hline Aromatic:Poslonizable-1.00 & $\begin{array}{l}\text { Number of pairs of aromatic-poslonazable } \\
\text { atoms within } 1 \text { bond }\end{array}$ & 0.008 \\
\hline Poslonizable:Poslonizable-5.00 & $\begin{array}{l}\text { Number of pairs of poslonazable-poslonazable } \\
\text { atoms within } 5 \text { bonds }\end{array}$ & 0.008 \\
\hline Donor:Poslonizable-2.00 & $\begin{array}{l}\text { Number of pairs of donor-poslonazable atoms } \\
\text { within } 2 \text { bonds }\end{array}$ & 0.008 \\
\hline fr_SH & Number of thiol groups & 0.007 \\
\hline fr_ester & Number of esters & 0.007 \\
\hline Neglonizable:Neglonizable-2.00 & $\begin{array}{l}\text { Number of pairs of neglonazable- } \\
\text { neglonazable atoms within } 2 \text { bonds }\end{array}$ & 0.007 \\
\hline Poslonizable:Poslonizable-1.00 & $\begin{array}{l}\text { Number of pairs of poslonazable-poslonazable } \\
\text { atoms within } 1 \text { bond }\end{array}$ & 0.006 \\
\hline Poslonizable:Poslonizable-6.00 & $\begin{array}{l}\text { Number of pairs of poslonazable-poslonazable } \\
\text { atoms within } 6 \text { bonds }\end{array}$ & 0.006 \\
\hline fr_ketone_Topliss & $\begin{array}{l}\text { Number of ketones excluding diaryl, a,b-unsat. } \\
\text { dienones, heteroatom on Calpha }\end{array}$ & 0.006 \\
\hline Neglonizable:Neglonizable-3.00 & $\begin{array}{l}\text { Number of pairs of neglonazable- } \\
\text { neglonazable atoms within } 3 \text { bonds }\end{array}$ & 0.006 \\
\hline Donor:Poslonizable-1.00 & $\begin{array}{l}\text { Number of pairs of donor-poslonazable atoms } \\
\text { within } 1 \text { bond }\end{array}$ & 0.005 \\
\hline Acceptor:Neglonizable-2.00 & $\begin{array}{l}\text { Number of pairs of acceptor-poslonazable } \\
\text { atoms within } 2 \text { bonds }\end{array}$ & 0.005 \\
\hline fr_hdrzine & Number of hydrazine groups & 0.005 \\
\hline Poslonizable:Poslonizable-2.00 & $\begin{array}{l}\text { Number of pairs of poslonazable-poslonazable } \\
\text { atoms within } 2 \text { bonds }\end{array}$ & 0.005 \\
\hline fr_thiophene & Number of thiophene rings & 0.005 \\
\hline Hydrophobe:Neglonizable-1.00 & $\begin{array}{l}\text { Number of pairs of hydrophobe-neglonazable } \\
\text { atoms within } 1 \text { bond }\end{array}$ & 0.005 \\
\hline fr_imidazole & Number of imidazole rings & 0.004 \\
\hline fr_C_S & Number of thiocarbonyl & 0.004 \\
\hline fr_urea & Number of urea groups & 0.004 \\
\hline
\end{tabular}




\begin{tabular}{|l|l|r|}
\hline fr_nitro_arom & Number of nitro benzene ring substituents & 0.004 \\
\hline Aromatic:Neglonizable-2.00 & $\begin{array}{l}\text { Number of pairs of aromatic-neglonazable } \\
\text { atoms within 2 bonds }\end{array}$ & 0.003 \\
\hline Acceptor:Poslonizable-1.00 & $\begin{array}{l}\text { Number of pairs of acceptor-poslonazable } \\
\text { atoms within 1 bond }\end{array}$ & 0.003 \\
\hline Aromatic:Neglonizable-3.00 & $\begin{array}{l}\text { Number of pairs of aromatic-neglonazable } \\
\text { atoms within 3 bonds }\end{array}$ & 0.002 \\
\hline Aromatic:Neglonizable-1.00 & $\begin{array}{l}\text { Number of pairs of aromatic-neglonazable } \\
\text { atoms within 1 bond }\end{array}$ & 0.002 \\
\hline fr_imide & Number of imide groups & 0.002 \\
\hline fr_nitro_arom_nonortho & $\begin{array}{l}\text { Number of non-ortho nitro benzene ring } \\
\text { substituents }\end{array}$ \\
\hline
\end{tabular}

Table S12. Feature importance for predictive model of PPI inhibitors targeting the Wdr5/MLL complex.

\begin{tabular}{|l|l|r|}
\hline Feature & Description & Importance \\
\hline Hydrophobe:Poslonizable-3.00 & $\begin{array}{l}\text { Number of pairs of hydrophobe- } \\
\text { poslonazable atoms within 3 bonds }\end{array}$ & 0.344 \\
\hline Aromatic:Poslonizable-6.00 & $\begin{array}{l}\text { Number of pairs of aromatic-poslonazable } \\
\text { atoms within 6 bonds }\end{array}$ & 0.270 \\
\hline Chi1v & Molecular connectivity index & 0.225 \\
\hline Acceptor:Poslonizable-3.00 & $\begin{array}{l}\text { Number of pairs of acceptor-neglonazable } \\
\text { atoms within 3 bonds }\end{array}$ & 0.160 \\
\hline
\end{tabular}

Table S13. Feature importance for predictive model of PPI inhibitors targeting the Xiap/Smac complex.

\begin{tabular}{|l|l|r|}
\hline Feature & & Importance \\
\hline Poslonizable_Count & Number of poslonazable atoms & 0.119 \\
\hline fr_amide & Number of amides & 0.087 \\
\hline Acceptor:Donor-6.00 & $\begin{array}{l}\text { Number of pairs of acceptor-donor atoms } \\
\text { within 6 bonds }\end{array}$ & 0.052 \\
\hline SlogP_VSA11 & $\begin{array}{l}\text { MOE-type descriptors, LogP and surface area } \\
\text { contributions }\end{array}$ & 0.035 \\
\hline Aromatic:Donor-5.00 & $\begin{array}{l}\text { Number of pairs of aromatic-donor atoms } \\
\text { within 5 bonds }\end{array}$ & 0.031 \\
\hline SMR_VSA9 & $\begin{array}{l}\text { MOE-type descriptors, molar refractivity and } \\
\text { surface area contributions }\end{array}$ & 0.027 \\
\hline VSA_EState8 & $\begin{array}{l}\text { MOE-type descriptors surface area } \\
\text { contributions and EState indices }\end{array}$ \\
\hline SMR_VSA10 & $\begin{array}{l}\text { MOE-type descriptors, molar refractivity and } \\
\text { surface area contributions }\end{array}$ & 0.026 \\
\hline Aromatic_Count & Number of atoms in aromatic rings & 0.024 \\
\hline Donor:Hydrophobe-2.00 & $\begin{array}{l}\text { Number of pairs of donor-hydrophobe atoms } \\
\text { within 2 bonds }\end{array}$ & 0.024 \\
\hline Acceptor:Acceptor-3.00 & $\begin{array}{l}\text { Number of pairs of acceptor-acceptor atoms } \\
\text { within 3 bonds }\end{array}$ & 0.020 \\
\hline Donor:Donor-2.00 & $\begin{array}{l}\text { Number of pairs of donor-donor atoms within } \\
\text { 2 bonds }\end{array}$ & 0.019 \\
\hline
\end{tabular}




\begin{tabular}{|c|c|c|}
\hline Poslonizable:Poslonizable-6.00 & $\begin{array}{l}\text { Number of pairs of poslonazable- } \\
\text { poslonazable atoms within } 6 \text { bonds }\end{array}$ & 0.016 \\
\hline NOCount & Number of Nitrogens and Oxygens & 0.016 \\
\hline fr_quatN & Number of quarternary nitrogens & 0.015 \\
\hline PEOE_VSA8 & $\begin{array}{l}\text { MOE-type descriptors, partial charges and } \\
\text { surface area contributions }\end{array}$ & 0.015 \\
\hline Neglonizable:Neglonizable-4.00 & $\begin{array}{l}\text { Number of pairs of neglonazable- } \\
\text { neglonazable atoms within } 4 \text { bonds }\end{array}$ & 0.015 \\
\hline Poslonizable:Poslonizable-3.00 & $\begin{array}{l}\text { Number of pairs of poslonazable- } \\
\text { poslonazable atoms within } 3 \text { bonds }\end{array}$ & 0.015 \\
\hline Poslonizable:Poslonizable-2.00 & $\begin{array}{l}\text { Number of pairs of poslonazable- } \\
\text { poslonazable atoms within } 2 \text { bonds }\end{array}$ & 0.015 \\
\hline Neglonizable:Neglonizable-1.00 & $\begin{array}{l}\text { Number of pairs of neglonazable- } \\
\text { neglonazable atoms within } 1 \text { bond }\end{array}$ & 0.014 \\
\hline Aromatic:Poslonizable-1.00 & $\begin{array}{l}\text { Number of pairs of aromatic-poslonazable } \\
\text { atoms within } 1 \text { bond }\end{array}$ & 0.014 \\
\hline Neglonizable:Neglonizable-2.00 & $\begin{array}{l}\text { Number of pairs of neglonazable- } \\
\text { neglonazable atoms within } 2 \text { bonds }\end{array}$ & 0.014 \\
\hline Hydrophobe:Poslonizable-4.00 & $\begin{array}{l}\text { Number of pairs of hydrophobe-poslonazable } \\
\text { atoms within } 4 \text { bonds }\end{array}$ & 0.014 \\
\hline Acceptor:Hydrophobe-5.00 & $\begin{array}{l}\text { Number of pairs of acceptor-hydrophobe } \\
\text { atoms within } 5 \text { bonds }\end{array}$ & 0.013 \\
\hline Aromatic:Poslonizable-3.00 & $\begin{array}{l}\text { Number of pairs of aromatic-poslonazable } \\
\text { atoms within } 3 \text { bonds }\end{array}$ & 0.013 \\
\hline Donor:Donor-3.00 & $\begin{array}{l}\text { Number of pairs of donor-donor atoms within } \\
3 \text { bonds }\end{array}$ & 0.013 \\
\hline Aromatic:Poslonizable-2.00 & $\begin{array}{l}\text { Number of pairs of aromatic-poslonazable } \\
\text { atoms within } 2 \text { bonds }\end{array}$ & 0.013 \\
\hline fr_Ar_N & Number of aromatic nitrogens & 0.012 \\
\hline Neglonizable:Neglonizable-3.00 & $\begin{array}{l}\text { Number of pairs of neglonazable- } \\
\text { neglonazable atoms within } 3 \text { bonds }\end{array}$ & 0.012 \\
\hline PEOE_VSA3 & $\begin{array}{l}\text { MOE-type descriptors, partial charges and } \\
\text { surface area contributions }\end{array}$ & 0.012 \\
\hline fr_Nhpyrrole & Number of $\mathrm{H}$-pyrrole nitrogens & 0.012 \\
\hline fr_Ar_OH & Number of aromatic hydroxyl groups & 0.011 \\
\hline Aromatic:Aromatic-3.00 & $\begin{array}{l}\text { Number of pairs of aromatic-aromatic atoms } \\
\text { within } 3 \text { bonds }\end{array}$ & 0.011 \\
\hline Poslonizable:Poslonizable-1.00 & $\begin{array}{l}\text { Number of pairs of poslonazable- } \\
\text { poslonazable atoms within } 1 \text { bond }\end{array}$ & 0.011 \\
\hline Donor:Hydrophobe-3.00 & $\begin{array}{l}\text { Number of pairs of donor-hydrophone atoms } \\
\text { within } 3 \text { bonds }\end{array}$ & 0.011 \\
\hline Acceptor:Hydrophobe-1.00 & $\begin{array}{l}\text { Number of pairs of acceptor-hydrophone } \\
\text { atoms within } 1 \text { bond }\end{array}$ & 0.011 \\
\hline Acceptor:Hydrophobe-4.00 & $\begin{array}{l}\text { Number of pairs of acceptor-hydrophone } \\
\text { atoms within } 4 \text { bonds }\end{array}$ & 0.011 \\
\hline Donor:Hydrophobe-4.00 & $\begin{array}{l}\text { Number of pairs of donor-hydrophone atoms } \\
\text { within } 4 \text { bonds }\end{array}$ & 0.011 \\
\hline Hydrophobe_Count & Number of hydrophobe atoms & 0.011 \\
\hline
\end{tabular}




\begin{tabular}{|c|c|c|}
\hline Hydrophobe:Hydrophobe- 4.00 & $\begin{array}{l}\text { Number of pairs of hydrophobe-hydrophone } \\
\text { atoms within } 4 \text { bonds }\end{array}$ & 0.010 \\
\hline Donor:Hydrophobe-1.00 & $\begin{array}{l}\text { Number of pairs of donor-hydrophone atoms } \\
\text { within } 1 \text { bond }\end{array}$ & 0.009 \\
\hline Aromatic:Aromatic-2.00 & $\begin{array}{l}\text { Number of pairs of aromatic-aromatic atoms } \\
\text { within } 2 \text { bonds }\end{array}$ & 0.009 \\
\hline Acceptor:Hydrophobe-3.00 & $\begin{array}{l}\text { Number of pairs of acceptor-hydrophone } \\
\text { atoms within } 3 \text { bonds }\end{array}$ & 0.009 \\
\hline Acceptor:Aromatic-2.00 & $\begin{array}{l}\text { Number of pairs of acceptor-aromatic atoms } \\
\text { within } 2 \text { bonds }\end{array}$ & 0.009 \\
\hline Acceptor:Hydrophobe-2.00 & $\begin{array}{l}\text { Number of pairs of acceptor-hydrophone } \\
\text { atoms within } 2 \text { bonds }\end{array}$ & 0.008 \\
\hline Aromatic:Aromatic-1.00 & $\begin{array}{l}\text { Number of pairs of aromatic-aromatic atoms } \\
\text { within } 1 \text { bond }\end{array}$ & 0.008 \\
\hline Aromatic:Hydrophobe-1.00 & $\begin{array}{l}\text { Number of pairs of aromatic-hydrophobe } \\
\text { atoms within } 1 \text { bond }\end{array}$ & 0.008 \\
\hline Aromatic:Hydrophobe-2.00 & $\begin{array}{l}\text { Number of pairs of aromatic-hydrophobe } \\
\text { atoms within } 2 \text { bonds }\end{array}$ & 0.008 \\
\hline Acceptor:Poslonizable-3.00 & $\begin{array}{l}\text { Number of pairs of aromatic-poslonazable } \\
\text { atoms within } 3 \text { bonds }\end{array}$ & 0.008 \\
\hline Hydrophobe:Hydrophobe-3.00 & $\begin{array}{l}\text { Number of pairs of hydrophobe-hydrophone } \\
\text { atoms within } 3 \text { bonds }\end{array}$ & 0.008 \\
\hline Hydrophobe:Hydrophobe-1.00 & $\begin{array}{l}\text { Number of pairs of hydrophobe-hydrophone } \\
\text { atoms within } 1 \text { bond }\end{array}$ & 0.007 \\
\hline Donor:Donor-1.00 & $\begin{array}{l}\text { Number of pairs of donor-donor atoms within } \\
1 \text { bond }\end{array}$ & 0.007 \\
\hline Acceptor:Neglonizable-2.00 & $\begin{array}{l}\text { Number of pairs of acceptor-neglonazable } \\
\text { atoms within } 2 \text { bonds }\end{array}$ & 0.007 \\
\hline fr_Ar_COO & Number of Aromatic carboxylic acide & 0.006 \\
\hline fr_oxazole & Number of oxazole rings & 0.006 \\
\hline Acceptor:Poslonizable-4.00 & $\begin{array}{l}\text { Number of pairs of acceptor-poslonazable } \\
\text { atoms within } 4 \text { bonds }\end{array}$ & 0.006 \\
\hline Hydrophobe:Neglonizable-3.00 & $\begin{array}{l}\text { Number of pairs of hydrophobe-neglonazable } \\
\text { atoms within } 3 \text { bonds }\end{array}$ & 0.004 \\
\hline Donor:Poslonizable-1.00 & $\begin{array}{l}\text { Number of pairs of donor-poslonazable atoms } \\
\text { within } 1 \text { bond }\end{array}$ & 0.004 \\
\hline Donor:Neglonizable-4.00 & $\begin{array}{l}\text { Number of pairs of donor-neglonazable atoms } \\
\text { within } 4 \text { bonds }\end{array}$ & 0.004 \\
\hline Hydrophobe:Poslonizable-2.00 & $\begin{array}{l}\text { Number of pairs of hydrophobe-poslonazable } \\
\text { atoms within } 2 \text { bonds }\end{array}$ & 0.004 \\
\hline Hydrophobe:Poslonizable-1.00 & $\begin{array}{l}\text { Number of pairs of hydrophobe-poslonazable } \\
\text { atoms within } 1 \text { bond }\end{array}$ & 0.003 \\
\hline Hydrophobe:Neglonizable-2.00 & $\begin{array}{l}\text { Number of pairs of hydrophobe-neglonazable } \\
\text { atoms within } 2 \text { bonds }\end{array}$ & 0.003 \\
\hline Hydrophobe:Neglonizable-1.00 & $\begin{array}{l}\text { Number of pairs of hydrophobe-neglonazable } \\
\text { atoms within } 1 \text { bond }\end{array}$ & 0.003 \\
\hline Acceptor:Poslonizable-2.00 & $\begin{array}{l}\text { Number of pairs of acceptor-poslonazable } \\
\text { atoms within } 2 \text { bonds }\end{array}$ & 0.003 \\
\hline Donor:Neglonizable-2.00 & Number of pairs of donor-poslonazable atoms & 0.002 \\
\hline
\end{tabular}




\begin{tabular}{|l|l|r|}
\hline & within 2 bonds & 0.002 \\
\hline fr_priamide & Number of primary amides & 0.002 \\
\hline Donor:Neglonizable-1.00 & $\begin{array}{l}\text { Number of pairs of donor-neglonazable atoms } \\
\text { within 1 bond }\end{array}$ & 0.001 \\
\hline Aromatic:Neglonizable-2.00 & $\begin{array}{l}\text { Number of pairs of aromatic-neglonazable } \\
\text { atoms within 2 bonds }\end{array}$ & 0.001 \\
\hline Aromatic:Neglonizable-3.00 & $\begin{array}{l}\text { Number of pairs of aromatic-neglonazable } \\
\text { atoms within 3 bonds }\end{array}$ & 0.001 \\
\hline fr_ketone_Topliss & $\begin{array}{l}\text { Number of ketones excluding diaryl, a,b- } \\
\text { unsat. dienones, heteroatom on Calpha }\end{array}$ & 0.001 \\
\hline fr_alkyl_carbamate & $\begin{array}{l}\text { Number of alkyl carbamates (subject to } \\
\text { hydrolysis) }\end{array}$ & 0.001 \\
\hline Aromatic:Neglonizable-1.00 & $\begin{array}{l}\text { Number of pairs of aromatic-neglonazable } \\
\text { atoms within 1 bond }\end{array}$ & \\
\hline
\end{tabular}

Table S14. Feature importance for predictive model of PPI inhibitors targeting the Annexin A2/S100A10 complex.

\begin{tabular}{|l|l|r|}
\hline Feature & Description & Importance \\
\hline Aromatic:Aromatic-4.00 & $\begin{array}{l}\text { Number of pairs of aromatic- } \\
\text { aromatic atoms within 4 bonds }\end{array}$ & 0.569 \\
\hline Kappa1 & Molecular shape index & 0.355 \\
\hline fr_piperdine & Number of piperdine rings & 0.076 \\
\hline
\end{tabular}

Table S15. Feature importance for predictive model of PPI inhibitors targeting the Brd2/Ack complex.

\begin{tabular}{|l|l|r|}
\hline Feature & Description & Importance \\
\hline Aromatic:Aromatic-4.00 & $\begin{array}{l}\text { Number of pairs of aromatic-aromatic atoms } \\
\text { within 4 bonds }\end{array}$ & 0.619 \\
\hline fr_Nhpyrrole & Number of H-pyrrole nitrogens & 0.121 \\
\hline Donor:Hydrophobe-2.00 & $\begin{array}{l}\text { Number of pairs of donor-hydrophobe atoms } \\
\text { within 2 bonds }\end{array}$ & 0.117 \\
\hline fr_morpholine & Number of morpholine rings & 0.031 \\
\hline Poslonizable:Poslonizable-3.00 & $\begin{array}{l}\text { Number of pairs of poslonazable- } \\
\text { poslonazable atoms within 3 bonds }\end{array}$ & 0.015 \\
\hline Acceptor:Poslonizable-1.00 & $\begin{array}{l}\text { Number of pairs of acceptor-poslonazable } \\
\text { atoms within 1 bond }\end{array}$ & 0.014 \\
\hline Neglonizable:Neglonizable-2.00 & $\begin{array}{l}\text { Number of pairs of neglonazable- } \\
\text { neglonazable atoms within 2 bonds }\end{array}$ \\
\hline Neglonizable:Neglonizable-1.00 & $\begin{array}{l}\text { Number of pairs of neglonazable- } \\
\text { neglonazable atoms within 1 bond }\end{array}$ & 0.014 \\
\hline Poslonizable:Poslonizable-2.00 & $\begin{array}{l}\text { Number of pairs of poslonazable- } \\
\text { poslonazable atoms within 2 bonds }\end{array}$ \\
\hline Poslonizable:Poslonizable-1.00 & $\begin{array}{l}\text { Number of pairs of poslonazable- } \\
\text { poslonazable atoms within 1 bond }\end{array}$ & 0.011 \\
\hline fr_alkyl_carbamate & $\begin{array}{l}\text { Number of alkyl carbamates (subject to } \\
\text { hydrolysis) }\end{array}$ & 0.008 \\
\hline Donor:Poslonizable-1.00 & Number of pairs of aromatic-aromatic atoms & 0.008 \\
\hline
\end{tabular}




\begin{tabular}{|l|l|r|}
\hline & within 1 bond & 0.007 \\
\hline Hydrophobe:Neglonizable-2.00 & $\begin{array}{l}\text { Number of pairs of aromatic-aromatic atoms } \\
\text { within 2 bonds }\end{array}$ & 0.005 \\
\hline Aromatic:Poslonizable-1.00 & $\begin{array}{l}\text { Number of pairs of aromatic-aromatic atoms } \\
\text { within 1 bond }\end{array}$ & 0.005 \\
\hline Acceptor:Neglonizable-1.00 & $\begin{array}{l}\text { Number of pairs of aromatic-aromatic atoms } \\
\text { within 1 bond }\end{array}$ & 0.004 \\
\hline Hydrophobe:Poslonizable-1.00 & $\begin{array}{l}\text { Number of pairs of aromatic-aromatic atoms } \\
\text { within 1 bond }\end{array}$ & 0.002 \\
\hline Donor:Neglonizable-1.00 & $\begin{array}{l}\text { Number of pairs of aromatic-aromatic atoms } \\
\text { within 1 bond }\end{array}$ & 0.001 \\
\hline Aromatic:Neglonizable-1.00 & $\begin{array}{l}\text { Number of pairs of aromatic-aromatic atoms } \\
\text { within 1 bond }\end{array}$ & \\
\hline
\end{tabular}

Table S16. Feature importance for predictive model of PPI inhibitors targeting the CD80/CD28 complex.

\begin{tabular}{|c|c|c|}
\hline Feature & Description & Importance \\
\hline Tox_30 & a1 a a a2 a 1 a a a a3 a 2 a a a a 3 & 0.200 \\
\hline Aromatic:Aromatic-1.00 & $\begin{array}{l}\text { Number of pairs of aromatic-aromatic atoms } \\
\text { within } 1 \text { bond }\end{array}$ & 0.082 \\
\hline fr_alkyl_halide & Number of alkyl halides & 0.081 \\
\hline Aromatic:Aromatic-3.00 & $\begin{array}{l}\text { Number of pairs of aromatic-aromatic atoms } \\
\text { within } 3 \text { bonds }\end{array}$ & 0.072 \\
\hline Aromatic:Donor-3.00 & $\begin{array}{l}\text { Number of pairs of aromatic-donor atoms within } \\
3 \text { bonds }\end{array}$ & 0.068 \\
\hline Aromatic:Aromatic-2.00 & $\begin{array}{l}\text { Number of pairs of aromatic-aromatic atoms } \\
\text { within } 2 \text { bonds }\end{array}$ & 0.064 \\
\hline Aromatic:Donor-2.00 & $\begin{array}{l}\text { Number of pairs of aromatic-donor atoms within } \\
2 \text { bonds }\end{array}$ & 0.056 \\
\hline Donor:Donor-1.00 & $\begin{array}{l}\text { Number of pairs of donor-donor atoms within } 1 \\
\text { bond }\end{array}$ & 0.050 \\
\hline Aromatic:Donor-1.00 & $\begin{array}{l}\text { Number of pairs of aromatic-donor atoms within } \\
1 \text { bond }\end{array}$ & 0.042 \\
\hline Acceptor:Aromatic-3.00 & $\begin{array}{l}\text { Number of pairs of acceptor-aromatic atoms } \\
\text { within } 3 \text { bonds }\end{array}$ & 0.035 \\
\hline Acceptor:Aromatic-2.00 & $\begin{array}{l}\text { Number of pairs of acceptor-aromatic atoms } \\
\text { within } 2 \text { bonds }\end{array}$ & 0.024 \\
\hline Donor:Donor-2.00 & $\begin{array}{l}\text { Number of pairs of donor-donor atoms within } 2 \\
\text { bonds }\end{array}$ & 0.023 \\
\hline Acceptor:Aromatic-1.00 & $\begin{array}{l}\text { Number of pairs of acceptor-aromatic atoms } \\
\text { within } 1 \text { bond }\end{array}$ & 0.017 \\
\hline Donor:Donor-3.00 & $\begin{array}{l}\text { Number of pairs of donor-donor atoms within } 3 \\
\text { bonds }\end{array}$ & 0.017 \\
\hline Aromatic:Hydrophobe-3.00 & $\begin{array}{l}\text { Number of pairs of aromatic-hydrophobe atoms } \\
\text { within } 3 \text { bonds }\end{array}$ & 0.015 \\
\hline Aromatic:Hydrophobe-1.00 & $\begin{array}{l}\text { Number of pairs of aromatic-hydrophobe atoms } \\
\text { within } 1 \text { bond }\end{array}$ & 0.013 \\
\hline Donor:Hydrophobe-2.00 & $\begin{array}{l}\text { Number of pairs of donor-hydrophobe atoms } \\
\text { within } 2 \text { bonds }\end{array}$ & 0.011 \\
\hline
\end{tabular}




\begin{tabular}{|c|c|c|}
\hline Aromatic:Hydrophobe-2.00 & $\begin{array}{l}\text { Number of pairs of aromatic-hydrophobe atoms } \\
\text { within } 2 \text { bonds }\end{array}$ & 0.011 \\
\hline Acceptor:Donor-3.00 & $\begin{array}{l}\text { Number of pairs of acceptor-donor atoms within } \\
3 \text { bonds }\end{array}$ & 0.011 \\
\hline Acceptor:Acceptor-2.00 & $\begin{array}{l}\text { Number of pairs of acceptor-acceptor atoms } \\
\text { within } 2 \text { bonds }\end{array}$ & 0.008 \\
\hline Hydrophobe:Hydrophobe-2.00 & $\begin{array}{l}\text { Number of pairs of hydrophobe-hydrophobe } \\
\text { atoms within } 2 \text { bonds }\end{array}$ & 0.007 \\
\hline Acceptor:Hydrophobe-2.00 & $\begin{array}{l}\text { Number of pairs of acceptor-hydrophobe atoms } \\
\text { within } 2 \text { bonds }\end{array}$ & 0.006 \\
\hline Acceptor:Hydrophobe-3.00 & $\begin{array}{l}\text { Number of pairs of acceptor-hydrophobe atoms } \\
\text { within } 3 \text { bonds }\end{array}$ & 0.006 \\
\hline Hydrophobe:Hydrophobe-1.00 & $\begin{array}{l}\text { Number of pairs of hydrophobe-hydrophobe } \\
\text { atoms within } 1 \text { bond }\end{array}$ & 0.005 \\
\hline Acceptor:Donor-1.00 & $\begin{array}{l}\text { Number of pairs of acceptor-donor atoms within } \\
1 \text { bond }\end{array}$ & 0.005 \\
\hline Acceptor:Acceptor-1.00 & $\begin{array}{l}\text { Number of pairs of acceptor-acceptor atoms } \\
\text { within } 1 \text { bond }\end{array}$ & 0.005 \\
\hline Hydrophobe:Hydrophobe-3.00 & $\begin{array}{l}\text { Number of pairs of hydrophobe-hydrophobe } \\
\text { atoms within } 3 \text { bonds }\end{array}$ & 0.005 \\
\hline Donor:Hydrophobe-3.00 & $\begin{array}{l}\text { Number of pairs of donor-hydrophobe atoms } \\
\text { within } 3 \text { bonds }\end{array}$ & 0.005 \\
\hline Acceptor:Acceptor-3.00 & $\begin{array}{l}\text { Number of pairs of acceptor-acceptor atoms } \\
\text { within } 3 \text { bonds }\end{array}$ & 0.005 \\
\hline Poslonizable:Poslonizable-1.00 & $\begin{array}{l}\text { Number of pairs of poslonazable-poslonazable } \\
\text { atoms within } 1 \text { bond }\end{array}$ & 0.004 \\
\hline Donor:Hydrophobe-1.00 & $\begin{array}{l}\text { Number of pairs of donor-hydrophobe atoms } \\
\text { within } 1 \text { bond }\end{array}$ & 0.004 \\
\hline Donor:Poslonizable-1.00 & $\begin{array}{l}\text { Number of pairs of donor-poslonazable atoms } \\
\text { within } 1 \text { bond }\end{array}$ & 0.003 \\
\hline Aromatic:Poslonizable-1.00 & $\begin{array}{l}\text { Number of pairs of aromatic-poslonazable } \\
\text { atoms within } 1 \text { bond }\end{array}$ & 0.003 \\
\hline Acceptor:Hydrophobe-1.00 & $\begin{array}{l}\text { Number of pairs of acceptor-hydrophobe atoms } \\
\text { within } 1 \text { bond }\end{array}$ & 0.003 \\
\hline Poslonizable:Poslonizable-2.00 & $\begin{array}{l}\text { Number of pairs of poslonazable-poslonazable } \\
\text { atoms within } 2 \text { bonds }\end{array}$ & 0.003 \\
\hline Hydrophobe:Poslonizable-3.00 & $\begin{array}{l}\text { Number of pairs of hydrophobe-poslonazable } \\
\text { atoms within } 3 \text { bonds }\end{array}$ & 0.003 \\
\hline Aromatic:Poslonizable-2.00 & $\begin{array}{l}\text { Number of pairs of aromatic-poslonazable } \\
\text { atoms within } 2 \text { bonds }\end{array}$ & 0.002 \\
\hline Acceptor:Donor-2.00 & $\begin{array}{l}\text { Number of pairs of acceptor-donor atoms within } \\
2 \text { bonds }\end{array}$ & 0.002 \\
\hline Poslonizable:Poslonizable-3.00 & $\begin{array}{l}\text { Number of pairs of poslonazable-poslonazable } \\
\text { atoms within } 3 \text { bonds }\end{array}$ & 0.002 \\
\hline Donor:Poslonizable-3.00 & $\begin{array}{l}\text { Number of pairs of donor-poslonazable atoms } \\
\text { within } 3 \text { bonds }\end{array}$ & 0.002 \\
\hline Aromatic:Poslonizable-3.00 & $\begin{array}{l}\text { Number of pairs of aromatic-poslonazable } \\
\text { atoms within } 3 \text { bonds }\end{array}$ & 0.002 \\
\hline Poslonizable:Poslonizable-4.00 & Number of pairs of poslonazable-poslonazable & 0.002 \\
\hline
\end{tabular}




\begin{tabular}{|l|l|r|}
\hline & atoms within 4 bonds & 0.001 \\
\hline Hydrophobe:Poslonizable-1.00 & $\begin{array}{l}\text { Number of pairs of hydrophobe-poslonazable } \\
\text { atoms within 1 bond }\end{array}$ & 0.001 \\
\hline Hydrophobe:Neglonizable-1.00 & $\begin{array}{l}\text { Number of pairs of hydrophobe-neglonazable } \\
\text { atoms within 1 bond }\end{array}$ & 0.001 \\
\hline Acceptor:Poslonizable-1.00 & $\begin{array}{l}\text { Number of pairs of acceptor-poslonazable } \\
\text { atoms within 1 bond }\end{array}$ & 0.001 \\
\hline Neglonizable:Neglonizable-2.00 & $\begin{array}{l}\text { Number of pairs of neglonazable-neglonazable } \\
\text { atoms within 2 bonds }\end{array}$ & 0.001 \\
\hline Hydrophobe:Poslonizable-2.00 & $\begin{array}{l}\text { Number of pairs of hydrophobe-poslonazable } \\
\text { atoms within 2 bonds }\end{array}$ & 0.001 \\
\hline Donor:Poslonizable-2.00 & $\begin{array}{l}\text { Number of pairs of donor-poslonazable atoms } \\
\text { within 2 bonds }\end{array}$ & 0.001 \\
\hline Donor:Neglonizable-2.00 & $\begin{array}{l}\text { Number of pairs of donor-neglonazable atoms } \\
\text { within 2 bonds }\end{array}$ & 0.001 \\
\hline Acceptor:Poslonizable-2.00 & $\begin{array}{l}\text { Number of pairs of acceptor-poslonazable } \\
\text { atoms within 2 bonds }\end{array}$ & 0.001 \\
\hline Donor:Neglonizable-3.00 & $\begin{array}{l}\text { Number of pairs of donor-neglonazable atoms } \\
\text { within 3 bonds }\end{array}$ \\
\hline Acceptor:Poslonizable-3.00 & $\begin{array}{l}\text { Number of pairs of acceptor-poslonazable } \\
\text { atoms within 3 bonds }\end{array}$ & 0.001 \\
\hline
\end{tabular}

Table S17. Feature importance for predictive model of PPI inhibitors targeting the IL2/IL2-R complex.

\begin{tabular}{|l|l|r|}
\hline Feature & Description & Importance \\
\hline PEOE_VSA11 & $\begin{array}{l}\text { MOE-type descriptors, partial charges and } \\
\text { surface area contributions }\end{array}$ & 0.595 \\
\hline Acceptor:Aromatic-4.00 & $\begin{array}{l}\text { Number of pairs of acceptor-aromatic atoms } \\
\text { within 4 bonds }\end{array}$ & 0.284 \\
\hline Neglonizable:Neglonizable-1.00 & $\begin{array}{l}\text { Number of pairs of neglonazable- } \\
\text { neglonazable atoms within 1 bond }\end{array}$ \\
\hline
\end{tabular}

Table S18. Feature importance for predictive model of PPI inhibitors targeting the Keap1/Nrf2 complex.

\begin{tabular}{|l|l|r|}
\hline Feature & Description & Importance \\
\hline Acceptor:Acceptor-1.00 & $\begin{array}{l}\text { Number of pairs of acceptor-acceptor atoms } \\
\text { within 1 bond }\end{array}$ & 0.192 \\
\hline Aromatic:Neglonizable-6.00 & $\begin{array}{l}\text { Number of pairs of aromatic-neglonazable } \\
\text { atoms within 6 bonds }\end{array}$ & 0.159 \\
\hline SMR_VSA6 & $\begin{array}{l}\text { MOE-type descriptors, molar refractivity and } \\
\text { surface area contributions }\end{array}$ & 0.110 \\
\hline Kappa3 & Molecular shape index & 0.098 \\
\hline Hydrophobe:Neglonizable-1.00 & $\begin{array}{l}\text { Number of pairs of hydrophobe-neglonazable } \\
\text { atoms within 1 bond }\end{array}$ & 0.091 \\
\hline Aromatic:Hydrophobe-6.00 & $\begin{array}{l}\text { Number of pairs of aromatic-hydrophobe atoms } \\
\text { within 6 bonds }\end{array}$ & 0.077 \\
\hline Hydrophobe:Hydrophobe-1.00 & $\begin{array}{l}\text { Number of pairs of hydrophobe-hydrophobe } \\
\text { atoms within 1 bond }\end{array}$ & 0.077 \\
\hline Donor:Hydrophobe-2.00 & Number of pairs of donor-hydrophobe atoms & 0.063 \\
\hline
\end{tabular}




\begin{tabular}{|l|l|r|}
\hline & within 2 bonds & 0.044 \\
\hline Aromatic:Neglonizable-2.00 & $\begin{array}{l}\text { Number of pairs of aromatic-neglonazable } \\
\text { atoms within 2 bonds }\end{array}$ & 0.031 \\
\hline fr_nitrile & Number of nitriles & 0.028 \\
\hline Donor:Neglonizable-1.00 & $\begin{array}{l}\text { Number of pairs of donor-neglonazable atoms } \\
\text { within 1 bond }\end{array}$ & 0.012 \\
\hline fr_tetrazole & Number of tetrazole rings & 0.005 \\
\hline Aromatic:Poslonizable-1.00 & $\begin{array}{l}\text { Number of pairs of aromatic-poslonazable } \\
\text { atoms within 1 bond }\end{array}$ & 0.004 \\
\hline Poslonizable:Poslonizable-2.00 & $\begin{array}{l}\text { Number of pairs of poslonazable-poslonazable } \\
\text { atoms within 2 bonds }\end{array}$ & 0.003 \\
\hline Poslonizable:Poslonizable-1.00 & $\begin{array}{l}\text { Number of pairs of poslonazable-poslonazable } \\
\text { atoms within 1 bond }\end{array}$ & 0.003 \\
\hline Hydrophobe:Poslonizable-1.00 & $\begin{array}{l}\text { Number of pairs of hydrophobe-poslonazable } \\
\text { atoms within 1 bond }\end{array}$ & 0.001 \\
\hline Donor:Poslonizable-1.00 & $\begin{array}{l}\text { Number of pairs of donor-poslonazable atoms } \\
\text { within 1 bond }\end{array}$ & 0.001 \\
\hline Acceptor:Poslonizable-1.00 & $\begin{array}{l}\text { Number of pairs of acceptor-poslonazable } \\
\text { atoms within 1 bond }\end{array}$ & \\
\hline
\end{tabular}

Table S19. Feature importance for predictive model of PPI inhibitors targeting the Menin/MLL complex.

\begin{tabular}{|l|l|r|}
\hline Feature & Descriptor & Importance \\
\hline SMR_VSA10 & $\begin{array}{l}\text { MOE-type descriptors, molar refractivity and surface } \\
\text { area contributions }\end{array}$ & 0.556 \\
\hline fr_nitrile & Number of nitriles & 0.399 \\
\hline SlogP_VSA7 & $\begin{array}{l}\text { MOE-type descriptors, LogP and surface area } \\
\text { contributions }\end{array}$ & 0.045 \\
\hline
\end{tabular}

Table S20. Feature importance for predictive model of PPI inhibitors targeting the STAT3 complex.

\begin{tabular}{|l|l|r|}
\hline Feature & Descriptor & Importance \\
\hline TPSA & Topological polar surface area & 0.588 \\
\hline SMR_VSA3 & $\begin{array}{l}\text { MOE-type descriptors, molar refractivity and } \\
\text { surface area contributions }\end{array}$ & 0.321 \\
\hline Acceptor:Poslonizable-1.00 & $\begin{array}{l}\text { Number of pairs of acceptor-poslonazable } \\
\text { atoms within 1 bond }\end{array}$ & 0.090 \\
\hline
\end{tabular}

Table S21. Feature importance for predictive model of PPI inhibitors targeting the TTR complex.

\begin{tabular}{|l|l|r|}
\hline Feature & Descriptor & Importance \\
\hline Chi1n & Molecular connectivity index & 0.776 \\
\hline SlogP_VSA7 & $\begin{array}{l}\text { MOE-type descriptors, LogP and surface area } \\
\text { contributions }\end{array}$ & 0.141 \\
\hline Donor:Neglonizable-1.00 & $\begin{array}{l}\text { Number of pairs of donor-neglonazable atoms } \\
\text { within 1 bond }\end{array}$ & 0.051 \\
\hline fr_hdrzine & Number of hydrazine groups & 0.032 \\
\hline
\end{tabular}

Table S22. Performance comparison of pdCSM-PPI and PPIM-pred on a non-redundant blind test. 


\begin{tabular}{|l|r|r|r|r|r|r|r|r|}
\hline & \multicolumn{4}{|c|}{ pdCSM-PPI } & \multicolumn{4}{c|}{ PPIM-pred } \\
\hline PPI target & F1 & AUC & Sensitivity & Specificity & F1 & AUC & Sensitivity & Specificity \\
\hline Bcl2-Like / Bak-Bax & 0.99 & 1.00 & 1.00 & 0.98 & 0.26 & $0.67^{+}$ & $0.71^{*}$ & $0.67^{*}$ \\
\hline Mdm2-Like / P53 & 0.97 & 1.00 & 0.97 & 0.96 & 0.45 & $0.84^{+}$ & $1.00^{*}$ & $0.79^{*}$ \\
\hline
\end{tabular}

${ }^{*} p$-value $<0.05$ by McNemar's Test compared to pdCSM-PPI

$+p$-value $<0.05$ by $z$ transformation test compared to pdCSM-PPI

Table S23. Performance comparison of pdCSM-PPI and SMMPPI on non-redundant blind tests.

\begin{tabular}{|l|r|r|r|r|r|r|}
\hline & \multicolumn{3}{|c|}{ pdCSM-PPI } & \multicolumn{3}{c}{ SMMPPI } \\
\hline PPI target & MCC & F1 & AUC & MCC & F1 & AUC \\
\hline Bcl2-Like / Bak-Bax & 0.86 & 0.92 & 0.92 & 0.70 & 0.84 & 0.92 \\
\hline Bromodomain / Histone & 0.82 & 0.90 & 0.90 & 0.73 & 0.87 & 0.93 \\
\hline LEDGF / IN & 0.70 & 0.85 & 0.85 & 0.70 & 0.85 & 0.97 \\
\hline LFA / ICAM & 1.00 & 1.00 & 1.00 & 0.92 & 0.96 & 0.99 \\
\hline Mdm2-Like / P53 & 0.88 & 0.93 & 0.93 & 0.79 & 0.89 & 0.93 \\
\hline Ras / SOS1 & 0.57 & 0.73 & 0.75 & 0.52 & 0.71 & 0.82 \\
\hline XIAP / Smac & 0.89 & 0.94 & 0.94 & 0.89 & 0.95 & 0.97 \\
\hline WDR5 / MLL & 0.92 & 0.96 & 0.94 & 0.74 & 0.87 & 0.87 \\
\hline CD4 / gp120 & 0.91 & 0.95 & 0.95 & 0.90 & 0.95 & 0.95 \\
\hline
\end{tabular}

Table S24. Distribution of compounds with experimentally determined inhibitory activity (IC50) across 45 different PPIs, on the original dataset retrieved from TIMBAL and IPPI-DB, and after clustering using the Butina algorithm with Tanimoto similarity cutoff of 0.8 . PPI targets with more than 40 inhibitors after clustering were further selected for building regression models.

\begin{tabular}{|l|r|r|}
\hline PPI & \# compounds & \# compounds (clustering 0.8) \\
\hline Integrins & 1606 & 907 \\
\hline Mdm2-Like / P53 & 685 & 400 \\
\hline LFA / ICAM & 277 & 150 \\
\hline BCL2-Like / BAX-BAK & 350 & 127 \\
\hline Bromodomain / Histone & 145 & 111 \\
\hline HIF-1a / p300 & 121 & 82 \\
\hline Cyclophilins & 105 & 73 \\
\hline LEDGF / IN & 74 & 68 \\
\hline XIAP / Smac & 69 & 42 \\
\hline CD80 / CD28 & 73 & 35 \\
\hline BRD2 / Ack & 47 & 32 \\
\hline Annexin A2/S100-A10 & 44 & 29 \\
\hline Neuropilin / VEGF & 41 & 24 \\
\hline FKBP1A/FK506 & 29 & 21 \\
\hline STAT3 & 38 & 21 \\
\hline TTR & 19 & 17 \\
\hline
\end{tabular}




\begin{tabular}{|l|r|r|}
\hline Transthyretin & 16 & 16 \\
\hline BRD4 / NUT & 16 & 15 \\
\hline BetaCatenin / Tcf4 and Tcf3 & 40 & 15 \\
\hline IL2 / IL-2R & 29 & 15 \\
\hline Rac1 & 15 & 15 \\
\hline Tubulin & 14 & 14 \\
\hline MENIN / MLL & 20 & 12 \\
\hline SPIN1 / H3 & 15 & 10 \\
\hline E2 / E1 & 10 & 8 \\
\hline MLLT1 / H3 & 16 & 8 \\
\hline PCNA trimer & 7 & 7 \\
\hline C-Myc / Max & 8 & 7 \\
\hline SOD1 & 10 & 5 \\
\hline RUNX1 / CBFb & 4 & 4 \\
\hline VEGF / VEGFR & 4 & 4 \\
\hline 53BP1 / H4 & 3 & 3 \\
\hline WD40 / H3 & 3 & 3 \\
\hline CRM1 / Rev & 2 & 1 \\
\hline NRP / VEGF & 1 & 1 \\
\hline Ras / SOS1 & 1 & 1 \\
\hline UPAR / UPA & 1 & 1 \\
\hline WDR5/MLL & 1 & 2 \\
\hline BRI1 & 2 & 2 \\
\hline CD4 / gp120 & 3 & 2 \\
\hline CD40 / CD154 & 1 & 1 \\
\hline CaM / CaMBD2 & 1 & 1 \\
\hline Rad51 & 1 & 1 \\
\hline TNFa / TNFa & 1 & 1 \\
\hline Tak1 / Tab1 & 1 & 1 \\
\hline
\end{tabular}

Table S25. Feature importance for the regression model of PPI inhibitors targeting the BCL2-Like / BAX-BAK complex.

\begin{tabular}{|l|l|r|}
\hline Feature & Description & Importance \\
\hline SMR_VSA5 & $\begin{array}{l}\text { MOE-type descriptors, molar refractivity and } \\
\text { surface area contributions }\end{array}$ & 0.128 \\
\hline SlogP_VSA7 & $\begin{array}{l}\text { MOE-type descriptors, LogP and surface area } \\
\text { contributions }\end{array}$ & 0.104 \\
\hline Chi2n & Molecular connectivity index & 0.096 \\
\hline fr_Ar_N & Number of aromatic nitrogens & 0.083 \\
\hline fr_bicyclic & Number of bicyclic structures & 0.042 \\
\hline Donor_Count & Number of hydrogen donors & 0.042 \\
\hline SMR_VSA10 & MOE-type descriptors, molar refractivity and & 0.037 \\
\hline
\end{tabular}




\begin{tabular}{|c|c|c|}
\hline & surface area contributions & \\
\hline VSA_EState8 & $\begin{array}{l}\text { MOE-type descriptors, surface area } \\
\text { contributions and EState indices }\end{array}$ & 0.036 \\
\hline SlogP_VSA10 & $\begin{array}{l}\text { MOE-type descriptors, LogP and surface area } \\
\text { contributions }\end{array}$ & 0.034 \\
\hline Donor:Donor-1.00 & $\begin{array}{l}\text { Number of pairs of donor-donor atoms within } \\
1 \text { bond }\end{array}$ & 0.032 \\
\hline Acceptor:Hydrophobe-3.00 & $\begin{array}{l}\text { Number of pairs of acceptor-hydrophobe } \\
\text { atoms within } 3 \text { bonds }\end{array}$ & 0.032 \\
\hline PEOE_VSA9 & $\begin{array}{l}\text { MOE-type descriptors, partial charges and } \\
\text { surface area contributions }\end{array}$ & 0.028 \\
\hline $\mathrm{fr} \_\mathrm{NH} 2$ & Number of Primary amines & 0.028 \\
\hline Aromatic_Count & Number of atoms in aromatic rings & 0.025 \\
\hline Acceptor:Hydrophobe-2.00 & $\begin{array}{l}\text { Number of pairs of acceptor-hydrophobe } \\
\text { atoms within } 2 \text { bonds }\end{array}$ & 0.024 \\
\hline Donor:Donor-6.00 & $\begin{array}{l}\text { Number of pairs of donor-donor atoms within } \\
6 \text { bonds }\end{array}$ & 0.023 \\
\hline Hydrophobe:Hydrophobe-4.00 & $\begin{array}{l}\text { Number of pairs of hydrophobe-hydrophobe } \\
\text { atoms within } 4 \text { bonds }\end{array}$ & 0.021 \\
\hline Donor:Hydrophobe-3.00 & $\begin{array}{l}\text { Number of pairs of donor-hydrophobe atoms } \\
\text { within } 3 \text { bonds }\end{array}$ & 0.021 \\
\hline SlogP_VSA8 & $\begin{array}{l}\text { MOE-type descriptors, LogP and surface area } \\
\text { contributions }\end{array}$ & 0.020 \\
\hline fr_thiazole & Number of thiazole rings & 0.016 \\
\hline SMR_VSA9 & $\begin{array}{l}\text { MOE-type descriptors, molar refractivity and } \\
\text { surface area contributions }\end{array}$ & 0.013 \\
\hline SlogP_VSA12 & $\begin{array}{l}\text { MOE-type descriptors, LogP and surface area } \\
\text { contributions }\end{array}$ & 0.010 \\
\hline fr_unbrch_alkane & $\begin{array}{l}\text { Number of unbranched alkanes of at least } 4 \\
\text { members (excludes halogenated alkanes) }\end{array}$ & 0.008 \\
\hline fr_alkyl_halide & Number of alkyl halides & 0.007 \\
\hline Acceptor:Poslonizable-4.00 & $\begin{array}{l}\text { Number of pairs of acceptor-poslonazable } \\
\text { atoms within } 4 \text { bonds }\end{array}$ & 0.007 \\
\hline Donor:Poslonizable-3.00 & $\begin{array}{l}\text { Number of pairs of donor-poslonazable atoms } \\
\text { within } 3 \text { bonds }\end{array}$ & 0.006 \\
\hline Poslonizable_Count & Number of poslonazable atoms & 0.006 \\
\hline Neglonizable:Neglonizable-2.00 & $\begin{array}{l}\text { Number of pairs of neglonazable- } \\
\text { neglonazable atoms within } 2 \text { bonds }\end{array}$ & 0.006 \\
\hline Poslonizable:Poslonizable-4.00 & $\begin{array}{l}\text { Number of pairs of poslonazable- } \\
\text { poslonazable atoms within } 4 \text { bonds }\end{array}$ & 0.005 \\
\hline Hydrophobe:Poslonizable-4.00 & $\begin{array}{l}\text { Number of pairs of hydrophobe-poslonazable } \\
\text { atoms within } 4 \text { bonds }\end{array}$ & 0.005 \\
\hline Donor:Hydrophobe-1.00 & $\begin{array}{l}\text { Number of pairs of donor-hydrophobe atoms } \\
\text { within } 1 \text { bond }\end{array}$ & 0.005 \\
\hline Hydrophobe:Poslonizable-3.00 & $\begin{array}{l}\text { Number of pairs of hydrophobe-poslonazable } \\
\text { atoms within } 3 \text { bonds }\end{array}$ & 0.004 \\
\hline Poslonizable:Poslonizable-3.00 & $\begin{array}{l}\text { Number of pairs of poslonazable- } \\
\text { poslonazable atoms within } 3 \text { bonds }\end{array}$ & 0.004 \\
\hline
\end{tabular}




\begin{tabular}{|c|c|c|}
\hline fr_sulfide & Number of thioether & 0.004 \\
\hline fr_Ar_OH & Number of aromatic hydroxyl groups & 0.003 \\
\hline Acceptor:Neglonizable-4.00 & $\begin{array}{l}\text { Number of pairs of acceptor-neglonazable } \\
\text { atoms within } 4 \text { bonds }\end{array}$ & 0.003 \\
\hline fr_pyridine & Number of pyridine rings & 0.003 \\
\hline Acceptor:Neglonizable-2.00 & $\begin{array}{l}\text { Number of pairs of acceptor-neglonazable } \\
\text { atoms within } 2 \text { bonds }\end{array}$ & 0.003 \\
\hline Neglonizable:Neglonizable-1.00 & $\begin{array}{l}\text { Number of pairs of neglonazable- } \\
\text { neglonazable atoms within } 1 \text { bond }\end{array}$ & 0.003 \\
\hline Donor:Donor-3.00 & $\begin{array}{l}\text { Number of pairs of donor-donor atoms within } \\
3 \text { bonds }\end{array}$ & 0.003 \\
\hline Neglonizable:Neglonizable-5.00 & $\begin{array}{l}\text { Number of pairs of neglonazable- } \\
\text { neglonazable atoms within } 5 \text { bonds }\end{array}$ & 0.002 \\
\hline Neglonizable:Neglonizable-4.00 & $\begin{array}{l}\text { Number of pairs of neglonazable- } \\
\text { neglonazable atoms within } 4 \text { bonds }\end{array}$ & 0.002 \\
\hline Acceptor:Neglonizable-1.00 & $\begin{array}{l}\text { Number of pairs of acceptor-neglonazable } \\
\text { atoms within } 1 \text { bond }\end{array}$ & 0.002 \\
\hline Donor:Neglonizable-1.00 & $\begin{array}{l}\text { Number of pairs of donor-neglonazable atoms } \\
\text { within } 1 \text { bond }\end{array}$ & 0.002 \\
\hline Hydrophobe:Poslonizable-1.00 & $\begin{array}{l}\text { Number of pairs of hydrophobe-poslonazable } \\
\text { atoms within } 1 \text { bond }\end{array}$ & 0.002 \\
\hline Hydrophobe:Neglonizable-4.00 & $\begin{array}{l}\text { Number of pairs of hydrophobe-neglonazable } \\
\text { atoms within } 4 \text { bonds }\end{array}$ & 0.002 \\
\hline Donor:Neglonizable-3.00 & $\begin{array}{l}\text { Number of pairs of donor-neglonazable atoms } \\
\text { within } 3 \text { bonds }\end{array}$ & 0.002 \\
\hline Donor:Neglonizable-5.00 & $\begin{array}{l}\text { Number of pairs of donor-neglonazable atoms } \\
\text { within } 5 \text { bonds }\end{array}$ & 0.002 \\
\hline fr_phenol & Number of phenols & 0.002 \\
\hline Acceptor:Poslonizable-1.00 & $\begin{array}{l}\text { Number of pairs of acceptor-poslonazable } \\
\text { atoms within } 1 \text { bond }\end{array}$ & 0.001 \\
\hline fr_ketone & Number of ketones & 0.001 \\
\hline Poslonizable:Poslonizable-2.00 & $\begin{array}{l}\text { Number of pairs of poslonazable- } \\
\text { poslonazable atoms within } 2 \text { bonds }\end{array}$ & 0.001 \\
\hline Aromatic:Neglonizable-4.00 & $\begin{array}{l}\text { Number of pairs of aromatic-neglonazable } \\
\text { atoms within } 4 \text { bonds }\end{array}$ & 0.001 \\
\hline Hydrophobe:Neglonizable-2.00 & $\begin{array}{l}\text { Number of pairs of hydrophobe-neglonazable } \\
\text { atoms within } 2 \text { bonds }\end{array}$ & 0.001 \\
\hline
\end{tabular}

Table S26. Feature importance for the regression model of PPI inhibitors targeting the Bromodomain / Histone complex.

\begin{tabular}{|l|l|r|}
\hline Feature & Description & Importance \\
\hline Chi1v & Molecular connectivity index & 0.219 \\
\hline BalabanJ & Balaban's connectivity topological index & 0.083 \\
\hline Kappa2 & Molecular shape index & 0.075 \\
\hline Tox_2 & a[NH2] & 0.054 \\
\hline PEOE_VSA7 & $\begin{array}{l}\text { MOE-type descriptors, partial charges and } \\
\text { surface area contributions }\end{array}$ & 0.039 \\
\hline
\end{tabular}




\begin{tabular}{|c|c|c|}
\hline PEOE_VSA9 & $\begin{array}{l}\text { MOE-type descriptors, partial charges and } \\
\text { surface area contributions }\end{array}$ & 0.039 \\
\hline Aromatic:Hydrophobe- 1.00 & $\begin{array}{l}\text { Number of pairs of aromatic-hydrophobe atoms } \\
\text { within } 1 \text { bond }\end{array}$ & 0.026 \\
\hline SMR_VSA4 & $\begin{array}{l}\text { MOE-type descriptors, molar refractivity and } \\
\text { surface area contributions }\end{array}$ & 0.025 \\
\hline Acceptor:Acceptor-5.00 & $\begin{array}{l}\text { Number of pairs of acceptor-acceptor atoms } \\
\text { within } 5 \text { bonds }\end{array}$ & 0.025 \\
\hline Hydrophobe:Hydrophobe-4.00 & $\begin{array}{l}\text { Number of pairs of hydrophobe-hydrophobe } \\
\text { atoms within } 4 \text { bonds }\end{array}$ & 0.024 \\
\hline Hydrophobe:Hydrophobe-1.00 & $\begin{array}{l}\text { Number of pairs of hydrophobe-hydrophobe } \\
\text { atoms within } 1 \text { bond }\end{array}$ & 0.022 \\
\hline Acceptor:Acceptor-1.00 & $\begin{array}{l}\text { Number of pairs of acceptor-acceptor atoms } \\
\text { within } 1 \text { bond }\end{array}$ & 0.020 \\
\hline PEOE_VSA2 & $\begin{array}{l}\text { MOE-type descriptors, partial charges and } \\
\text { surface area contributions }\end{array}$ & 0.018 \\
\hline Aromatic:Hydrophobe-5.00 & $\begin{array}{l}\text { Number of pairs of aromatic-hydrophobe atoms } \\
\text { within } 5 \text { bonds }\end{array}$ & 0.018 \\
\hline fr_NHO & Number of Tertiary amines & 0.017 \\
\hline fr_Nhpyrrole & Number of $\mathrm{H}$-pyrrole nitrogens & 0.017 \\
\hline VSA_EState10 & $\begin{array}{l}\text { MOE-type descriptors surface area contributions } \\
\text { and EState indices }\end{array}$ & 0.017 \\
\hline fr_benzene & Number of benzene rings & 0.015 \\
\hline fr_Imine & Number of Imines & 0.014 \\
\hline SMR_VSA6 & $\begin{array}{l}\text { MOE-type descriptors, molar refractivity and } \\
\text { surface area contributions }\end{array}$ & 0.014 \\
\hline Aromatic_Count & Number of atoms in aromatic rings & 0.013 \\
\hline SlogP_VSA10 & $\begin{array}{l}\text { MOE-type descriptors, LogP and surface area } \\
\text { contributions }\end{array}$ & 0.011 \\
\hline SlogP_VSA1 & $\begin{array}{l}\text { MOE-type descriptors, LogP and surface area } \\
\text { contributions }\end{array}$ & 0.011 \\
\hline SMR_VSA5 & $\begin{array}{l}\text { MOE-type descriptors, molar refractivity and } \\
\text { surface area contributions }\end{array}$ & 0.011 \\
\hline fr_aniline & Number of anilines & 0.011 \\
\hline Aromatic:Aromatic-5.00 & $\begin{array}{l}\text { Number of pairs of aromatic-aromatic atoms } \\
\text { within } 5 \text { bonds }\end{array}$ & 0.010 \\
\hline Acceptor:Aromatic-2.00 & $\begin{array}{l}\text { Number of pairs of acceptor-aromatic atoms } \\
\text { within } 2 \text { bonds }\end{array}$ & 0.010 \\
\hline VSA_EState8 & $\begin{array}{l}\text { MOE-type descriptors, surface area } \\
\text { contributions and EState indices }\end{array}$ & 0.009 \\
\hline Acceptor:Aromatic-5.00 & $\begin{array}{l}\text { Number of pairs of acceptor-aromatic atoms } \\
\text { within } 5 \text { bonds }\end{array}$ & 0.009 \\
\hline Acceptor:Aromatic-4.00 & $\begin{array}{l}\text { Number of pairs of acceptor-aromatic atoms } \\
\text { within } 4 \text { bonds }\end{array}$ & 0.009 \\
\hline PEOE_VSA3 & $\begin{array}{l}\text { MOE-type descriptors, partial charges and } \\
\text { surface area contributions }\end{array}$ & 0.009 \\
\hline fr_aryl_methyl & Number of aryl methyl sites for hydroxylation & 0.009 \\
\hline Donor:Hydrophobe-4.00 & Number of pairs of donor-hydrophobe atoms & 0.008 \\
\hline
\end{tabular}




\begin{tabular}{|c|c|c|}
\hline & within 4 bonds & \\
\hline Aromatic:Donor-3.00 & $\begin{array}{l}\text { Number of pairs of aromatic-donor atoms within } \\
3 \text { bonds }\end{array}$ & 0.007 \\
\hline SlogP_VSA5 & $\begin{array}{l}\text { MOE-type descriptors, LogP and surface area } \\
\text { contributions }\end{array}$ & 0.007 \\
\hline SMR_VSA10 & $\begin{array}{l}\text { MOE-type descriptors, molar refractivity and } \\
\text { surface area contributions }\end{array}$ & 0.006 \\
\hline fr_thiophene & Number of thiophene rings & 0.006 \\
\hline Acceptor:Poslonizable-6.00 & $\begin{array}{l}\text { Number of pairs of acceptor-poslonazable } \\
\text { atoms within } 6 \text { bonds }\end{array}$ & 0.006 \\
\hline PEOE_VSA11 & $\begin{array}{l}\text { MOE-type descriptors, partial charges and } \\
\text { surface area contributions }\end{array}$ & 0.006 \\
\hline PEOE_VSA5 & $\begin{array}{l}\text { MOE-type descriptors, partial charges and } \\
\text { surface area contributions }\end{array}$ & 0.006 \\
\hline Aromatic:Poslonizable-6.00 & $\begin{array}{l}\text { Number of pairs of aromatic-poslonazable } \\
\text { atoms within } 6 \text { bonds }\end{array}$ & 0.005 \\
\hline Aromatic:Donor-1.00 & $\begin{array}{l}\text { Number of pairs of aromatic-donor atoms within } \\
1 \text { bond }\end{array}$ & 0.005 \\
\hline PEOE_VSA4 & $\begin{array}{l}\text { MOE-type descriptors, partial charges and } \\
\text { surface area contributions }\end{array}$ & 0.004 \\
\hline Aromatic:Poslonizable-5.00 & $\begin{array}{l}\text { Number of pairs of aromatic-poslonazable } \\
\text { atoms within } 5 \text { bonds }\end{array}$ & 0.004 \\
\hline fr_Ar_OH & Number of aromatic hydroxyl groups & 0.003 \\
\hline Donor:Hydrophobe-1.00 & $\begin{array}{l}\text { Number of pairs of donor-hydrophobe atoms } \\
\text { within } 1 \text { bond }\end{array}$ & 0.003 \\
\hline Donor:Donor-6.00 & $\begin{array}{l}\text { Number of pairs of donor-donor atoms within } 6 \\
\text { bonds }\end{array}$ & 0.002 \\
\hline Donor:Donor-3.00 & $\begin{array}{l}\text { Number of pairs of donor-donor atoms within } 3 \\
\text { bonds }\end{array}$ & 0.002 \\
\hline Donor:Donor-2.00 & $\begin{array}{l}\text { Number of pairs of donor-donor atoms within } 2 \\
\text { bonds }\end{array}$ & 0.002 \\
\hline Poslonizable_Count & Number of poslonazable atoms & 0.002 \\
\hline fr_halogen & Number of halogens & 0.002 \\
\hline Donor:Donor-4.00 & $\begin{array}{l}\text { Number of pairs of donor-donor atoms within } 4 \\
\text { bonds }\end{array}$ & 0.002 \\
\hline Neglonizable_Count & Number of neglonazable atoms & 0.001 \\
\hline Acceptor:Poslonizable-2.00 & $\begin{array}{l}\text { Number of pairs of acceptor-poslonazable } \\
\text { atoms within } 2 \text { bonds }\end{array}$ & 0.001 \\
\hline Donor:Donor-5.00 & $\begin{array}{l}\text { Number of pairs of donor-donor atoms within } 5 \\
\text { bonds }\end{array}$ & 0.001 \\
\hline Hydrophobe:Poslonizable-3.00 & $\begin{array}{l}\text { Number of pairs of hydrophobe-poslonazable } \\
\text { atoms within } 3 \text { bonds }\end{array}$ & 0.001 \\
\hline Donor:Neglonizable-1.00 & $\begin{array}{l}\text { Number of pairs of donor-neglonazable atoms } \\
\text { within } 1 \text { bond }\end{array}$ & 0.001 \\
\hline Donor:Poslonizable-4.00 & $\begin{array}{l}\text { Number of pairs of donor-poslonazable atoms } \\
\text { within } 4 \text { bonds }\end{array}$ & 0.001 \\
\hline Aromatic:Poslonizable-4.00 & $\begin{array}{l}\text { Number of pairs of aromatic-poslonazable } \\
\text { atoms within } 4 \text { bonds }\end{array}$ & 0.001 \\
\hline
\end{tabular}




\begin{tabular}{|l|l|r|}
\hline Poslonizable:Poslonizable-5.00 & $\begin{array}{l}\text { Number of pairs of poslonazable-poslonazable } \\
\text { atoms within 5 bonds }\end{array}$ & 0.001 \\
\hline Acceptor:Poslonizable-3.00 & $\begin{array}{l}\text { Number of pairs of acceptor-poslonazable } \\
\text { atoms within 3 bonds }\end{array}$ & 0.001 \\
\hline
\end{tabular}

Table S27. Feature importance for the regression model of PPI inhibitors targeting the HIF-1a / p300 complex.

\begin{tabular}{|l|l|r|}
\hline Feature & Description & Importance \\
\hline SlogP_VSA8 & $\begin{array}{l}\text { MOE-type descriptors, LogP and surface area } \\
\text { contributions }\end{array}$ & 0.464 \\
\hline RingCount & Number of rings & 0.192 \\
\hline SMR_VSA3 & $\begin{array}{l}\text { MOE-type descriptors, molar refractivity and } \\
\text { surface area contributions }\end{array}$ & 0.09 \\
\hline Acceptor:Donor-1.00 & $\begin{array}{l}\text { Number of pairs of acceptor-donor atoms } \\
\text { within 1 bond }\end{array}$ & 0.076 \\
\hline fr_Al_OH & Number of aliphatic hydroxyl groups & 0.044 \\
\hline Donor:Poslonizable-6.00 & $\begin{array}{l}\text { Number of pairs of donor-poslonazable atoms } \\
\text { within 6 bonds }\end{array}$ & 0.044 \\
\hline VSA_EState10 & $\begin{array}{l}\text { MOE-type descriptors, surface area } \\
\text { contributions and EState indices }\end{array}$ \\
\hline Aromatic:Poslonizable-1.00 & $\begin{array}{l}\text { Number of pairs of aromatic-poslonazable } \\
\text { atoms within 1 bond }\end{array}$ & 0.041 \\
\hline Donor:Donor-1.00 & $\begin{array}{l}\text { Number of pairs of donor-donor atoms within } \\
1 \text { bond }\end{array}$ & 0.012 \\
\hline Acceptor:Poslonizable-1.00 & $\begin{array}{l}\text { Number of pairs of acceptor-poslonazable } \\
\text { atoms within 1 bond }\end{array}$ & 0.006 \\
\hline Hydrophobe:Poslonizable-1.00 & $\begin{array}{l}\text { Number of pairs of hydrophobe-poslonazable } \\
\text { atoms within 1 bond }\end{array}$ & 0.004 \\
\hline Neglonizable:Neglonizable-1.00 & $\begin{array}{l}\text { Number of pairs of neglonazable- } \\
\text { neglonazable atoms within 1 bond }\end{array}$ & 0.001 \\
\hline Neglonizable:Neglonizable-2.00 & $\begin{array}{l}\text { Number of pairs of neglonazable- } \\
\text { neglonazable atoms within 1 bonds }\end{array}$ \\
\hline
\end{tabular}

Table S28. Feature importance for the regression model of PPI inhibitors targeting the Mdm2-Like / P53 complex.

\begin{tabular}{|l|l|r|}
\hline Feature & Description & Importance \\
\hline PEOE_VSA13 & $\begin{array}{l}\text { MOE-type descriptors, partial charges and } \\
\text { surface area contributions }\end{array}$ & 0.058 \\
\hline Chi4n & Molecular connectivity index & 0.054 \\
\hline VSA_EState8 & $\begin{array}{l}\text { MOE-type descriptors, surface area } \\
\text { contributions and EState indices }\end{array}$ & 0.051 \\
\hline fr_halogen & Number of halogens & 0.046 \\
\hline VSA_EState10 & $\begin{array}{l}\text { MOE-type descriptors, surface area } \\
\text { contributions and EState indices }\end{array}$ & 0.035 \\
\hline PEOE_VSA9 & MOE-type descriptors, partial charges and & 0.035 \\
\hline
\end{tabular}




\begin{tabular}{|c|c|c|}
\hline & surface area contributions & \\
\hline Donor:Donor-6.00 & $\begin{array}{l}\text { Number of pairs of donor-donor atoms within } 6 \\
\text { bonds }\end{array}$ & 0.031 \\
\hline fr_bicyclic & Number of bicyclic structures & 0.030 \\
\hline fr_NH1 & Number of Secondary amines & 0.029 \\
\hline ChiOn & Molecular connectivity index & 0.026 \\
\hline BalabanJ & Balaban's connectivity topological index & 0.023 \\
\hline Hydrophobe:Neglonizable-3.00 & $\begin{array}{l}\text { Number of pairs of hydrophobe-neglonazable } \\
\text { atoms within } 3 \text { bonds }\end{array}$ & 0.023 \\
\hline Chi3n & Molecular connectivity index & 0.023 \\
\hline PEOE_VSA11 & $\begin{array}{l}\text { MOE-type descriptors, partial charges and } \\
\text { surface area contributions }\end{array}$ & 0.021 \\
\hline VSA_EState9 & $\begin{array}{l}\text { MOE-type descriptors surface area contributions } \\
\text { and EState indices }\end{array}$ & 0.020 \\
\hline Kappa1 & Molecular shape index & 0.018 \\
\hline Donor_Count & Number of hydrogen donors & 0.018 \\
\hline HeavyAtomCount & Number of heavy atoms & 0.018 \\
\hline fr_aniline & Number of anilines & 0.017 \\
\hline FCount & Number of fluorine atoms & 0.016 \\
\hline MolWt & Molecular weight & 0.015 \\
\hline PEOE_VSA8 & $\begin{array}{l}\text { MOE-type descriptors, partial charges and } \\
\text { surface area contributions }\end{array}$ & 0.015 \\
\hline SMR_VSA7 & $\begin{array}{l}\text { MOE-type descriptors, molar refractivity and } \\
\text { surface area contributions }\end{array}$ & 0.014 \\
\hline Acceptor:Hydrophobe-4.00 & $\begin{array}{l}\text { Number of pairs of acceptor-hydrophobe atoms } \\
\text { within } 4 \text { bonds }\end{array}$ & 0.012 \\
\hline Donor:Donor-4.00 & $\begin{array}{l}\text { Number of pairs of donor-donor atoms within } 4 \\
\text { bonds }\end{array}$ & 0.012 \\
\hline Donor:Hydrophobe-4.00 & $\begin{array}{l}\text { Number of pairs of donor-hydrophobe atoms } \\
\text { within } 4 \text { bonds }\end{array}$ & 0.012 \\
\hline fr_NH2 & Number of Primary amines & 0.011 \\
\hline fr_C_O_noCOO & Number of carbonyl $\mathrm{O}$, excluding $\mathrm{COOH}$ & 0.011 \\
\hline Acceptor:Hydrophobe-2.00 & $\begin{array}{l}\text { Number of pairs of acceptor-hydrophobe atoms } \\
\text { within } 2 \text { bonds }\end{array}$ & 0.011 \\
\hline Acceptor:Donor-1.00 & $\begin{array}{l}\text { Number of pairs of acceptor-donor atoms within } \\
1 \text { bond }\end{array}$ & 0.011 \\
\hline SMR_VSA1 & $\begin{array}{l}\text { MOE-type descriptors, molar refractivity and } \\
\text { surface area contributions }\end{array}$ & 0.011 \\
\hline fr_C_O & Number of carbonyl O & 0.011 \\
\hline Donor:Neglonizable-1.00 & $\begin{array}{l}\text { Number of pairs of donor-neglonazable atoms } \\
\text { within } 1 \text { bond }\end{array}$ & 0.010 \\
\hline Acceptor:Acceptor-3.00 & $\begin{array}{l}\text { Number of pairs of acceptor-acceptor atoms } \\
\text { within } 3 \text { bonds }\end{array}$ & 0.010 \\
\hline Acceptor:Aromatic-1.00 & $\begin{array}{l}\text { Number of pairs of acceptor-aromatic atoms } \\
\text { within } 1 \text { bond }\end{array}$ & 0.010 \\
\hline $\mathrm{fr} \_\mathrm{HOCCN}$ & Number of $\mathrm{C}(\mathrm{OH}) \mathrm{CCN}$-Ctert-alkyl or & 0.009 \\
\hline
\end{tabular}




\begin{tabular}{|c|c|c|}
\hline & $\mathrm{C}(\mathrm{OH}) \mathrm{CCN}$ cyclic & \\
\hline Donor:Donor-3.00 & $\begin{array}{l}\text { Number of pairs of donor-donor atoms within } 3 \\
\text { bonds }\end{array}$ & 0.009 \\
\hline Acceptor:Hydrophobe- 6.00 & $\begin{array}{l}\text { Number of pairs of acceptor-hydrophobe atoms } \\
\text { within } 6 \text { bonds }\end{array}$ & 0.009 \\
\hline Aromatic:Aromatic-1.00 & $\begin{array}{l}\text { Number of pairs of aromatic-aromatic atoms } \\
\text { within } 1 \text { bond }\end{array}$ & 0.008 \\
\hline Aromatic:Aromatic-2.00 & $\begin{array}{l}\text { Number of pairs of aromatic-aromatic atoms } \\
\text { within } 2 \text { bonds }\end{array}$ & 0.008 \\
\hline Donor:Poslonizable-1.00 & $\begin{array}{l}\text { Number of pairs of donor-poslonazable atoms } \\
\text { within } 1 \text { bond }\end{array}$ & 0.008 \\
\hline PEOE_VSA14 & $\begin{array}{l}\text { MOE-type descriptors, partial charges and } \\
\text { surface area contributions }\end{array}$ & 0.008 \\
\hline fr_aryl_methyl & Number of aryl methyl sites for hydroxylation & 0.008 \\
\hline Acceptor:Aromatic-3.00 & $\begin{array}{l}\text { Number of pairs of acceptor-aromatic atoms } \\
\text { within } 3 \text { bonds }\end{array}$ & 0.008 \\
\hline SMR_VSA9 & $\begin{array}{l}\text { MOE-type descriptors, molar refractivity and } \\
\text { surface area contributions }\end{array}$ & 0.007 \\
\hline SlogP_VSA8 & $\begin{array}{l}\text { MOE-type descriptors, LogP and surface area } \\
\text { contributions }\end{array}$ & 0.006 \\
\hline Neglonizable:Neglonizable-2.00 & $\begin{array}{l}\text { Number of pairs of neglonazable-neglonazable } \\
\text { atoms within } 2 \text { bonds }\end{array}$ & 0.006 \\
\hline Donor:Poslonizable-5.00 & $\begin{array}{l}\text { Number of pairs of donor-poslonazable atoms } \\
\text { within } 5 \text { bonds }\end{array}$ & 0.006 \\
\hline Neglonizable:Neglonizable-3.00 & $\begin{array}{l}\text { Number of pairs of neglonazable-neglonazable } \\
\text { atoms within } 3 \text { bonds }\end{array}$ & 0.006 \\
\hline fr_Ndealkylation1 & Number of XCCNR groups & 0.006 \\
\hline fr_Nhpyrrole & Number of $\mathrm{H}$-pyrrole nitrogens & 0.006 \\
\hline Aromatic:Aromatic-4.00 & $\begin{array}{l}\text { Number of pairs of aromatic-aromatic atoms } \\
\text { within } 4 \text { bonds }\end{array}$ & 0.005 \\
\hline Aromatic:Aromatic-5.00 & $\begin{array}{l}\text { Number of pairs of aromatic-aromatic atoms } \\
\text { within } 5 \text { bonds }\end{array}$ & 0.005 \\
\hline Hydrophobe:Poslonizable-2.00 & $\begin{array}{l}\text { Number of pairs of hydrophobe-poslonazable } \\
\text { atoms within } 2 \text { bonds }\end{array}$ & 0.005 \\
\hline Aromatic:Aromatic-6.00 & $\begin{array}{l}\text { Number of pairs of aromatic-aromatic atoms } \\
\text { within } 6 \text { bonds }\end{array}$ & 0.005 \\
\hline Donor:Neglonizable-2.00 & $\begin{array}{l}\text { Number of pairs of donor-neglonazable atoms } \\
\text { within s bonds }\end{array}$ & 0.005 \\
\hline Acceptor:Poslonizable-5.00 & $\begin{array}{l}\text { Number of pairs of acceptor-poslonazable atoms } \\
\text { within } 5 \text { bonds }\end{array}$ & 0.005 \\
\hline Donor:Donor-2.00 & $\begin{array}{l}\text { Number of pairs of donor-donor atoms within } 2 \\
\text { bonds }\end{array}$ & 0.005 \\
\hline Aromatic_Count & Number of atoms in aromatic rings & 0.005 \\
\hline fr_piperzine & Number of piperzine rings & 0.004 \\
\hline Neglonizable:Neglonizable-6.00 & $\begin{array}{l}\text { Number of pairs of neglonazable-neglonazable } \\
\text { atoms within } 6 \text { bonds }\end{array}$ & 0.004 \\
\hline Donor:Poslonizable-3.00 & $\begin{array}{l}\text { Number of pairs of donor-poslonazable atoms } \\
\text { within } 3 \text { bonds }\end{array}$ & 0.004 \\
\hline
\end{tabular}




\begin{tabular}{|c|c|c|}
\hline fr_pyridine & Number of pyridine rings & 0.004 \\
\hline Neglonizable:Neglonizable-1.00 & $\begin{array}{l}\text { Number of pairs of neglonazable-neglonazable } \\
\text { atoms within } 1 \text { bond }\end{array}$ & 0.004 \\
\hline Acceptor:Poslonizable-1.00 & $\begin{array}{l}\text { Number of pairs of acceptor-poslonazable atoms } \\
\text { within } 1 \text { bonds }\end{array}$ & 0.004 \\
\hline Hydrophobe:Poslonizable-1.00 & $\begin{array}{l}\text { Number of pairs of hydrophobe-poslonazable } \\
\text { atoms within } 1 \text { bond }\end{array}$ & 0.004 \\
\hline Poslonizable:Poslonizable-3.00 & $\begin{array}{l}\text { Number of pairs of poslonazable-poslonazable } \\
\text { atoms within } 3 \text { bonds }\end{array}$ & 0.004 \\
\hline fr_ArN & $\begin{array}{l}\text { Number of } \mathrm{N} \text { functional groups attached to } \\
\text { aromatics }\end{array}$ & 0.004 \\
\hline Aromatic:Poslonizable-3.00 & $\begin{array}{l}\text { Number of pairs of aromatic-poslonazable atoms } \\
\text { within } 3 \text { bonds }\end{array}$ & 0.004 \\
\hline Hydrophobe:Poslonizable-3.00 & $\begin{array}{l}\text { Number of pairs of hydrophobe-poslonazable } \\
\text { atoms within } 3 \text { bonds }\end{array}$ & 0.004 \\
\hline fr_nitro & Number of nitro groups & 0.003 \\
\hline fr_morpholine & Number of morpholine rings & 0.003 \\
\hline Hydrophobe:Neglonizable-1.00 & $\begin{array}{l}\text { Number of pairs of hydrophobe-neglonazable } \\
\text { atoms within } 1 \text { bond }\end{array}$ & 0.003 \\
\hline Tox_1 & $\mathrm{O}=\mathrm{N}(\sim \mathrm{O}) \mathrm{a}$ & 0.003 \\
\hline fr_nitro_arom & Number of nitro benzene ring substituents & 0.003 \\
\hline fr_imide & Number of imide groups & 0.002 \\
\hline Acceptor:Neglonizable-1.00 & $\begin{array}{l}\text { Number of pairs of acceptor-neglonazable } \\
\text { atoms within } 1 \text { bond }\end{array}$ & 0.002 \\
\hline Hydrophobe:Poslonizable-4.00 & $\begin{array}{l}\text { Number of pairs of hydrophobe-poslonazable } \\
\text { atoms within } 4 \text { bonds }\end{array}$ & 0.002 \\
\hline Aromatic:Neglonizable-6.00 & $\begin{array}{l}\text { Number of pairs of aromatic-neglonazable } \\
\text { atoms within } 63 \text { bonds }\end{array}$ & 0.002 \\
\hline fr_nitrile & Number of nitriles & 0.002 \\
\hline fr_thiophene & Number of thiophene rings & 0.001 \\
\hline fr_N_O & Number of hydroxylamine groups & 0.001 \\
\hline Aromatic:Neglonizable-2.00 & $\begin{array}{l}\text { Number of pairs of aromatic-neglonazable } \\
\text { atoms within } 2 \text { bonds }\end{array}$ & 0.001 \\
\hline Aromatic:Neglonizable-4.00 & $\begin{array}{l}\text { Number of pairs of aromatic-neglonazable } \\
\text { atoms within } 4 \text { bonds }\end{array}$ & 0.001 \\
\hline Aromatic:Neglonizable-3.00 & $\begin{array}{l}\text { Number of pairs of aromatic-neglonazable } \\
\text { atoms within } 3 \text { bonds }\end{array}$ & 0.001 \\
\hline
\end{tabular}

Table S29. Feature importance for the regression model of PPI inhibitors targeting the Integrins complex.

\begin{tabular}{|l|l|r|}
\hline Feature & Description & Importance \\
\hline fr_Al_COO & Number of aliphatic carboxylic acids & 0.067 \\
\hline SlogP_VSA5 & $\begin{array}{l}\text { MOE-type descriptors, LogP and surface area } \\
\text { contributions }\end{array}$ & 0.059 \\
\hline fr_pyridine & Number of pyridine rings & 0.055 \\
\hline
\end{tabular}




\begin{tabular}{|c|c|c|}
\hline Hydrophobe:Hydrophobe-4.00 & $\begin{array}{l}\text { Number of pairs of hydrophobe-hydrophobe } \\
\text { atoms within } 4 \text { bonds }\end{array}$ & 0.049 \\
\hline MolWt & Molecular weight & 0.044 \\
\hline SlogP_VSA2 & $\begin{array}{l}\text { MOE-type descriptors, LogP and surface area } \\
\text { contributions }\end{array}$ & 0.042 \\
\hline Acceptor:Aromatic-5.00 & $\begin{array}{l}\text { Number of pairs of acceptor-aromatic atoms } \\
\text { within } 5 \text { bonds }\end{array}$ & 0.040 \\
\hline HallKierAlpha & Hall-Kier alpha value & 0.038 \\
\hline Aromatic_Count & Number of atoms in aromatic rings & 0.034 \\
\hline Aromatic:Aromatic-1.00 & $\begin{array}{l}\text { Number of pairs of aromatic-aromatic atoms } \\
\text { within } 1 \text { bond }\end{array}$ & 0.033 \\
\hline Acceptor:Acceptor-6.00 & $\begin{array}{l}\text { Number of pairs of acceptor-acceptor atoms } \\
\text { within } 6 \text { bonds }\end{array}$ & 0.033 \\
\hline fr_NH1 & Number of Secondary amines & 0.032 \\
\hline fr_piperdine & Number of piperdine rings & 0.030 \\
\hline SlogP_VSA4 & $\begin{array}{l}\text { MOE-type descriptors, LogP and surface area } \\
\text { contributions }\end{array}$ & 0.027 \\
\hline PEOE_VSA2 & $\begin{array}{l}\text { MOE-type descriptors, partial charges and } \\
\text { surface area contributions }\end{array}$ & 0.026 \\
\hline PEOE_VSA12 & $\begin{array}{l}\text { MOE-type descriptors, partial charges and } \\
\text { surface area contributions }\end{array}$ & 0.026 \\
\hline PEOE_VSA14 & $\begin{array}{l}\text { MOE-type descriptors, partial charges and } \\
\text { surface area contributions }\end{array}$ & 0.023 \\
\hline Donor:Donor-6.00 & $\begin{array}{l}\text { Number of pairs of donor-donor atoms within } \\
6 \text { bonds }\end{array}$ & 0.023 \\
\hline Donor:Donor-5.00 & $\begin{array}{l}\text { Number of pairs of donor-donor atoms within } \\
5 \text { bonds }\end{array}$ & 0.021 \\
\hline Hydrophobe:Neglonizable-2.00 & $\begin{array}{l}\text { Number of pairs of hydrophobe-neglonazable } \\
\text { atoms within } 2 \text { bonds }\end{array}$ & 0.018 \\
\hline Donor:Donor-4.00 & $\begin{array}{l}\text { Number of pairs of donor-donor atoms within } \\
4 \text { bonds }\end{array}$ & 0.018 \\
\hline PEOE_VSA13 & $\begin{array}{l}\text { MOE-type descriptors, partial charges and } \\
\text { surface area contributions }\end{array}$ & 0.018 \\
\hline Acceptor:Acceptor-1.00 & $\begin{array}{l}\text { Number of pairs of acceptor-acceptor atoms } \\
\text { within } 1 \text { bond }\end{array}$ & 0.018 \\
\hline Hydrophobe:Neglonizable-3.00 & $\begin{array}{l}\text { Number of pairs of hydrophobe-neglonazable } \\
\text { atoms within } 3 \text { bonds }\end{array}$ & 0.017 \\
\hline Hydrophobe:Neglonizable-4.00 & $\begin{array}{l}\text { Number of pairs of hydrophobe-neglonazable } \\
\text { atoms within } 4 \text { bonds }\end{array}$ & 0.017 \\
\hline Donor:Donor-3.00 & $\begin{array}{l}\text { Number of pairs of donor-donor atoms within } \\
3 \text { bonds }\end{array}$ & 0.014 \\
\hline Donor:Donor-2.00 & $\begin{array}{l}\text { Number of pairs of donor-donor atoms within } \\
2 \text { bonds }\end{array}$ & 0.013 \\
\hline fr_amidine & Number of amidine groups & 0.012 \\
\hline Donor:Neglonizable-2.00 & $\begin{array}{l}\text { Number of pairs of donor-neglonazable atoms } \\
\text { within } 2 \text { bonds }\end{array}$ & 0.012 \\
\hline fr_sulfonamd & Number of sulfonamides & 0.011 \\
\hline Hydrophobe:Poslonizable-5.00 & Number of pairs of hydrophober-poslonazable & 0.011 \\
\hline
\end{tabular}




\begin{tabular}{|c|c|c|}
\hline & atoms within 5 bonds & \\
\hline Acceptor:Poslonizable-6.00 & $\begin{array}{l}\text { Number of pairs of acceptor-poslonazable } \\
\text { atoms within } 6 \text { bonds }\end{array}$ & 0.010 \\
\hline fr_Imine & Number of Imines & 0.009 \\
\hline Poslonizable:Poslonizable-6.00 & $\begin{array}{l}\text { Number of pairs of poslonazable- } \\
\text { poslonazable atoms within } 6 \text { bonds }\end{array}$ & 0.008 \\
\hline fr_alkyl_halide & Number of alkyl halides & 0.007 \\
\hline fr_Ndealkylation1 & Number of XCCNR groups & 0.007 \\
\hline Aromatic:Poslonizable-6.00 & $\begin{array}{l}\text { Number of pairs of aromatic-poslonazable } \\
\text { atoms within } 6 \text { bonds }\end{array}$ & 0.007 \\
\hline Donor:Poslonizable-6.00 & $\begin{array}{l}\text { Number of pairs of donor-poslonazable atoms } \\
\text { within } 6 \text { bonds }\end{array}$ & 0.006 \\
\hline Donor:Poslonizable-5.00 & $\begin{array}{l}\text { Number of pairs of donor-poslonazable atoms } \\
\text { within } 5 \text { bonds }\end{array}$ & 0.006 \\
\hline Poslonizable:Poslonizable-5.00 & $\begin{array}{l}\text { Number of pairs of poslonazable- } \\
\text { poslonazable atoms within } 5 \text { bonds }\end{array}$ & 0.006 \\
\hline Neglonizable:Poslonizable-6.00 & $\begin{array}{l}\text { Number of pairs of neglonazable- } \\
\text { poslonazable atoms within } 6 \text { bonds }\end{array}$ & 0.005 \\
\hline FCount & Number of fluorine atoms & 0.005 \\
\hline fr_ArN & $\begin{array}{l}\text { Number of } \mathrm{N} \text { functional groups attached to } \\
\text { aromatics }\end{array}$ & 0.005 \\
\hline Donor:Poslonizable-4.00 & $\begin{array}{l}\text { Number of pairs of donor-poslonazable atoms } \\
\text { within } 4 \text { bonds }\end{array}$ & 0.004 \\
\hline Poslonizable:Poslonizable-3.00 & $\begin{array}{l}\text { Number of pairs of poslonazable- } \\
\text { poslonazable atoms within } 3 \text { bonds }\end{array}$ & 0.004 \\
\hline Poslonizable:Poslonizable-2.00 & $\begin{array}{l}\text { Number of pairs of poslonazable- } \\
\text { poslonazable atoms within } 2 \text { bonds }\end{array}$ & 0.004 \\
\hline Acceptor:Poslonizable-4.00 & $\begin{array}{l}\text { Number of pairs of acceptor-poslonazable } \\
\text { atoms within } 4 \text { bonds }\end{array}$ & 0.004 \\
\hline Acceptor:Poslonizable-1.00 & $\begin{array}{l}\text { Number of pairs of acceptor-poslonazable } \\
\text { atoms within } 1 \text { bond }\end{array}$ & 0.003 \\
\hline Donor:Poslonizable-2.00 & $\begin{array}{l}\text { Number of pairs of donor-poslonazable atoms } \\
\text { within } 2 \text { bonds }\end{array}$ & 0.003 \\
\hline Poslonizable:Poslonizable-1.00 & $\begin{array}{l}\text { Number of pairs of poslonazable- } \\
\text { poslonazable atoms within } 1 \text { bond }\end{array}$ & 0.003 \\
\hline Aromatic:Poslonizable-2.00 & $\begin{array}{l}\text { Number of pairs of aromatic-poslonazable } \\
\text { atoms within } 2 \text { bonds }\end{array}$ & 0.002 \\
\hline Neglonizable:Poslonizable-2.00 & $\begin{array}{l}\text { Number of pairs of neglonazable- } \\
\text { poslonazable atoms within } 2 \text { bonds }\end{array}$ & 0.002 \\
\hline fr_nitrile & Number of nitriles & 0.002 \\
\hline fr_imide & Number of imide groups & 0.002 \\
\hline fr_barbitur & Number of barbiturate groups & 0.001 \\
\hline Hydrophobe:Poslonizable-1.00 & $\begin{array}{l}\text { Number of pairs of hydrophobe-poslonazable } \\
\text { atoms within } 1 \text { bond }\end{array}$ & 0.001 \\
\hline Aromatic:Poslonizable-1.00 & $\begin{array}{l}\text { Number of pairs of aromatic-poslonazable } \\
\text { atoms within } 1 \text { bond }\end{array}$ & 0.001 \\
\hline fr_term_acetylene & Number of terminal acetylenes & 0.001 \\
\hline
\end{tabular}


Table S30. Feature importance for the regression model of PPI inhibitors targeting the LFA / ICAM complex.

\begin{tabular}{|l|l|r|}
\hline Feature & Description & Importance \\
\hline Hydrophobe:Hydrophobe-1.00 & $\begin{array}{l}\text { Number of pairs of hydrophobe- } \\
\text { hydrophobe atoms within 1 bond }\end{array}$ & 0.149 \\
\hline VSA_EState8 & $\begin{array}{l}\text { MOE-type descriptors surface area } \\
\text { contributions and EState indices }\end{array}$ & 0.145 \\
\hline Kappa2 & Molecular shape index & 0.093 \\
\hline SlogP_VSA10 & $\begin{array}{l}\text { MOE-type descriptors, LogP and surface } \\
\text { area contributions }\end{array}$ & 0.091 \\
\hline SlogP_VSA5 & $\begin{array}{l}\text { MOE-type descriptors, LogP and surface } \\
\text { area contributions }\end{array}$ & 0.091 \\
\hline fr_bicyclic & Number of bicyclic structures & 0.082 \\
\hline SMR_VSA5 & $\begin{array}{l}\text { MOE-type descriptors, molar refractivity } \\
\text { and surface area contributions }\end{array}$ & 0.078 \\
\hline Acceptor:Donor-4.00 & $\begin{array}{l}\text { Number of pairs of acceptor-donor atoms } \\
\text { within 4 bonds }\end{array}$ & 0.072 \\
\hline fr_para_hydroxylation & Number of para-hydroxylation sites & 0.068 \\
\hline fr_urea & Number of urea groups & 0.043 \\
\hline fr_imide & Number of imide groups & 0.040 \\
\hline fr_amide & Number of amides & 0.035 \\
\hline Donor:Donor-1.00 & $\begin{array}{l}\text { Number of pairs of donor-donor atoms } \\
\text { within 1 bond }\end{array}$ & 0.012 \\
\hline
\end{tabular}

Table S31. Feature importance for the regression model of PPI inhibitors targeting the Cyclophilins complex.

\begin{tabular}{|l|l|r|}
\hline Feature & Description & Importance \\
\hline Aromatic:Poslonizable-1.00 & $\begin{array}{l}\text { Number of pairs of aromatic-poslonazable } \\
\text { atoms within 1 bond }\end{array}$ & 0.176 \\
\hline Poslonizable:Poslonizable-2.00 & $\begin{array}{l}\text { Number of pairs of poslonazable- } \\
\text { posglonazable atoms within 2 bonds }\end{array}$ & 0.135 \\
\hline Poslonizable:Poslonizable-1.00 & $\begin{array}{l}\text { Number of pairs of poslonazable- } \\
\text { poslonazable atoms within 1 bond }\end{array}$ & 0.113 \\
\hline Hydrophobe:Poslonizable-2.00 & $\begin{array}{l}\text { Number of pairs of hydrophobe- } \\
\text { poslonazable atoms within 2 bonds }\end{array}$ & 0.078 \\
\hline Donor:Poslonizable-1.00 & $\begin{array}{l}\text { Number of pairs of donor-poslonazable } \\
\text { atoms within 1 bond }\end{array}$ & 0.075 \\
\hline SMR_VSA3 & $\begin{array}{l}\text { MOE-type descriptors, molar refractivity and } \\
\text { surface area contributions }\end{array}$ \\
\hline Donor:Poslonizable-2.00 & $\begin{array}{l}\text { Number of pairs of donor-poslonazable } \\
\text { atoms within 2 bonds }\end{array}$ & 0.070 \\
\hline Acceptor:Acceptor-4.00 & $\begin{array}{l}\text { Number of pairs of acceptor-acceptor atoms } \\
\text { within 4 bonds }\end{array}$ \\
\hline Acceptor:Acceptor-2.00 & $\begin{array}{l}\text { Number of pairs of acceptor-acceptor atoms } \\
\text { within 2 bonds }\end{array}$ \\
\hline
\end{tabular}




\begin{tabular}{|c|c|c|}
\hline Donor:Donor-5.00 & $\begin{array}{l}\text { Number of pairs of donor-donor atoms within } \\
5 \text { bonds }\end{array}$ & 0.040 \\
\hline SMR_VSA6 & $\begin{array}{l}\text { MOE-type descriptors, molar refractivity and } \\
\text { surface area contributions }\end{array}$ & 0.019 \\
\hline Chi1n & Molecular connectivity index & 0.019 \\
\hline fr_amide & Number of amides & 0.015 \\
\hline Acceptor:Donor-1.00 & $\begin{array}{l}\text { Number of pairs of acceptor-donor atoms } \\
\text { within } 1 \text { bond }\end{array}$ & 0.012 \\
\hline Donor:Hydrophobe-1.00 & $\begin{array}{l}\text { Number of pairs of donor-hydrophobe atoms } \\
\text { within } 1 \text { bond }\end{array}$ & 0.009 \\
\hline Aromatic:Donor-1.00 & $\begin{array}{l}\text { Number of pairs of aromatic-donor atoms } \\
\text { within } 1 \text { bond }\end{array}$ & 0.009 \\
\hline SMR_VSA10 & $\begin{array}{l}\text { MOE-type descriptors, molar refractivity and } \\
\text { surface area contributions }\end{array}$ & 0.008 \\
\hline Hydrophobe:Hydrophobe-1.00 & $\begin{array}{l}\text { Number of pairs of hydrophobe-hydrophobe } \\
\text { atoms within } 1 \text { bond }\end{array}$ & 0.008 \\
\hline Acceptor:Acceptor-1.00 & $\begin{array}{l}\text { Number of pairs of acceptor-acceptor atoms } \\
\text { within } 1 \text { bond }\end{array}$ & 0.006 \\
\hline Hydrophobe:Hydrophobe-2.00 & $\begin{array}{l}\text { Number of pairs of hydrophobe-hydrophobe } \\
\text { atoms within } 2 \text { bonds }\end{array}$ & 0.005 \\
\hline Aromatic:Hydrophobe-1.00 & $\begin{array}{l}\text { Number of pairs of aromatic-hydrophobe } \\
\text { atoms within } 1 \text { bond }\end{array}$ & 0.004 \\
\hline Aromatic:Aromatic-1.00 & $\begin{array}{l}\text { Number of pairs of aromatic-aromatic atoms } \\
\text { within } 1 \text { bond }\end{array}$ & 0.004 \\
\hline Aromatic:Aromatic-3.00 & $\begin{array}{l}\text { Number of pairs of aromatic-aromatic atoms } \\
\text { within } 3 \text { bonds }\end{array}$ & 0.004 \\
\hline Aromatic:Hydrophobe-2.00 & $\begin{array}{l}\text { Number of pairs of aromatic-hydrophobe } \\
\text { atoms within } 2 \text { bonds }\end{array}$ & 0.004 \\
\hline Aromatic:Aromatic-2.00 & $\begin{array}{l}\text { Number of pairs of aromatic-aromatic atoms } \\
\text { within } 2 \text { bonds }\end{array}$ & 0.004 \\
\hline fr_benzene & Number of benzene rings & 0.003 \\
\hline Acceptor:Hydrophobe-2.00 & $\begin{array}{l}\text { Number of pairs of acceptor-hydrophobe } \\
\text { atoms within } 2 \text { bonds }\end{array}$ & 0.003 \\
\hline fr_pyridine & Number of pyridine rings & 0.001 \\
\hline fr_Ar_N & Number of aromatic nitrogens & 0.001 \\
\hline
\end{tabular}

Table S32. Feature importance for the regression model of PPI inhibitors targeting the LEDGF / IN complex.

\begin{tabular}{|l|l|r|}
\hline Feature & Description & Importance \\
\hline SlogP_VSA8 & $\begin{array}{l}\text { MOE-type descriptors, LogP and surface area } \\
\text { contributions }\end{array}$ & 0.132 \\
\hline Acceptor:Acceptor-5.00 & $\begin{array}{l}\text { Number of pairs of acceptor-acceptor atoms } \\
\text { within 5 bonds }\end{array}$ & 0.065 \\
\hline PEOE_VSA10 & $\begin{array}{l}\text { MOE-type descriptors, partial charges and } \\
\text { surface area contributions }\end{array}$ & 0.059 \\
\hline SlogP_VSA2 & $\begin{array}{l}\text { MOE-type descriptors, LogP and surface area } \\
\text { contributions }\end{array}$ \\
\hline
\end{tabular}




\begin{tabular}{|c|c|c|}
\hline PEOE_VSA6 & $\begin{array}{l}\text { MOE-type descriptors, partial charges and } \\
\text { surface area contributions }\end{array}$ & 0.039 \\
\hline Kappa1 & Molecular shape index & 0.034 \\
\hline PEOE_VSA7 & $\begin{array}{l}\text { MOE-type descriptors, partial charges and } \\
\text { surface area contributions }\end{array}$ & 0.029 \\
\hline SlogP_VSA4 & $\begin{array}{l}\text { MOE-type descriptors, LogP and surface area } \\
\text { contributions }\end{array}$ & 0.027 \\
\hline Chi1v & Molecular connectivity index & 0.027 \\
\hline PEOE_VSA5 & $\begin{array}{l}\text { MOE-type descriptors, partial charges and } \\
\text { surface area contributions }\end{array}$ & 0.027 \\
\hline ChiOv & Molecular connectivity index & 0.026 \\
\hline Chi2n & Molecular connectivity index & 0.025 \\
\hline PEOE_VSA2 & $\begin{array}{l}\text { MOE-type descriptors, partial charges and } \\
\text { surface area contributions }\end{array}$ & 0.024 \\
\hline Donor:Donor-3.00 & $\begin{array}{l}\text { Number of pairs of donor-donor atoms within } 3 \\
\text { bonds }\end{array}$ & 0.022 \\
\hline fr_nitro_arom & Number of nitro benzene ring substituents & 0.021 \\
\hline Donor:Neglonizable-4.00 & $\begin{array}{l}\text { Number of pairs of donor-neglonazable atoms } \\
\text { within } 4 \text { bonds }\end{array}$ & 0.021 \\
\hline fr_C_O_noCOO & Number of carbonyl $\mathrm{O}$, excluding $\mathrm{COOH}$ & 0.021 \\
\hline SMR_VSA3 & $\begin{array}{l}\text { MOE-type descriptors, molar refractivity and } \\
\text { surface area contributions }\end{array}$ & 0.020 \\
\hline Acceptor:Hydrophobe-2.00 & $\begin{array}{l}\text { Number of pairs of acceptor-hydrophobe atoms } \\
\text { within } 2 \text { bonds }\end{array}$ & 0.020 \\
\hline Chio & Molecular connectivity index & 0.019 \\
\hline Tox_1 & $\mathrm{O}=\mathrm{N}(\sim \mathrm{O}) \mathrm{a}$ & 0.019 \\
\hline Donor:Donor-5.00 & $\begin{array}{l}\text { Number of pairs of donor-donor atoms within } 5 \\
\text { bonds }\end{array}$ & 0.018 \\
\hline fr_nitro & Number of nitro groups & 0.018 \\
\hline fr_hdrzone & Number of hydrazone groups & 0.018 \\
\hline Donor:Donor-6.00 & $\begin{array}{l}\text { Number of pairs of donor-donor atoms within } 6 \\
\text { bonds }\end{array}$ & 0.017 \\
\hline Donor:Hydrophobe-5.00 & $\begin{array}{l}\text { Number of pairs of donor-hydrophobe atoms } \\
\text { within } 5 \text { bonds }\end{array}$ & 0.017 \\
\hline Donor:Neglonizable-3.00 & $\begin{array}{l}\text { Number of pairs of donor-neglonazable atoms } \\
\text { within } 3 \text { bonds }\end{array}$ & 0.016 \\
\hline HallKierAlpha & Hall-Kier alpha value & 0.015 \\
\hline Acceptor:Donor-2.00 & $\begin{array}{l}\text { Number of pairs of acceptor-donor atoms within } 2 \\
\text { bonds }\end{array}$ & 0.015 \\
\hline Aromatic:Donor-3.00 & $\begin{array}{l}\text { Number of pairs of aromatic-donor atoms within } 3 \\
\text { bonds }\end{array}$ & 0.014 \\
\hline Donor:Donor-1.00 & $\begin{array}{l}\text { Number of pairs of donor-donor atoms within } 1 \\
\text { bond }\end{array}$ & 0.012 \\
\hline PEOE_VSA12 & $\begin{array}{l}\text { MOE-type descriptors, partial charges and } \\
\text { surface area contributions }\end{array}$ & 0.012 \\
\hline Aromatic:Hydrophobe-4.00 & $\begin{array}{l}\text { Number of pairs of aromatic-hydrophobe atoms } \\
\text { within } 4 \text { bonds }\end{array}$ & 0.010 \\
\hline
\end{tabular}




\begin{tabular}{|c|c|c|}
\hline Acceptor:Donor-5.00 & $\begin{array}{l}\text { Number of pairs of acceptor-donor atoms within } 5 \\
\text { bonds }\end{array}$ & 0.010 \\
\hline fr_pyridine & Number of pyridine rings & 0.010 \\
\hline Acceptor:Aromatic-4.00 & $\begin{array}{l}\text { Number of pairs of acceptor-aromatic atoms } \\
\text { within } 4 \text { bonds }\end{array}$ & 0.010 \\
\hline Hydrophobe:Hydrophobe- 3.00 & $\begin{array}{l}\text { Number of pairs of hydrophobe-hydrophobe } \\
\text { atoms within } 3 \text { bonds }\end{array}$ & 0.009 \\
\hline fr_ArN & $\begin{array}{l}\text { Number of } \mathrm{N} \text { functional groups attached to } \\
\text { aromatics }\end{array}$ & 0.007 \\
\hline Acceptor:Hydrophobe-5.00 & $\begin{array}{l}\text { Number of pairs of acceptor-hydrophobe atoms } \\
\text { within } 5 \text { bonds }\end{array}$ & 0.007 \\
\hline SlogP_VSA7 & $\begin{array}{l}\text { MOE-type descriptors, LogP and surface area } \\
\text { contributions }\end{array}$ & 0.007 \\
\hline Acceptor:Hydrophobe-4.00 & $\begin{array}{l}\text { Number of pairs of acceptor-hydrophobe atoms } \\
\text { within } 4 \text { bonds }\end{array}$ & 0.006 \\
\hline fr_amide & Number of amides & 0.005 \\
\hline Tox_2 & $\mathrm{a}[\mathrm{NH} 2]$ & 0.003 \\
\hline Poslonizable:Poslonizable-1.00 & $\begin{array}{l}\text { Number of pairs of poslonazable-poslonazable } \\
\text { atoms within } 1 \text { bond }\end{array}$ & 0.003 \\
\hline Donor:Donor-2.00 & $\begin{array}{l}\text { Number of pairs of donor-donor atoms within } 2 \\
\text { bonds }\end{array}$ & 0.003 \\
\hline Poslonizable:Poslonizable-2.00 & $\begin{array}{l}\text { Number of pairs of poslonazable-poslonazable } \\
\text { atoms within } 2 \text { bonds }\end{array}$ & 0.002 \\
\hline Tox_12 & {$[\mathrm{OH}, \mathrm{NH} 2][\mathrm{N}, \mathrm{O}]$} & 0.002 \\
\hline fr_furan & Number of furan rings & 0.002 \\
\hline Donor:Poslonizable-1.00 & $\begin{array}{l}\text { Number of pairs of donor-poslonazable atoms } \\
\text { within } 1 \text { bond }\end{array}$ & 0.002 \\
\hline fr_azo & Number of azo groups & 0.002 \\
\hline fr_ketone & Number of ketones & 0.002 \\
\hline Poslonizable:Poslonizable-3.00| & $\begin{array}{l}\text { Number of pairs of poslonazable-poslonazable } \\
\text { atoms within } 3 \text { bonds }\end{array}$ & 0.002 \\
\hline Aromatic:Poslonizable-1.00 & $\begin{array}{l}\text { Number of pairs of aromatic-poslonazable atoms } \\
\text { within } 1 \text { bond }\end{array}$ & 0.002 \\
\hline fr_thiazole & Number of thiazole rings & 0.002 \\
\hline fr_unbrch_alkane & $\begin{array}{l}\text { Number of unbranched alkanes of at least } 4 \\
\text { members (excludes halogenated alkanes) }\end{array}$ & 0.001 \\
\hline fr_C_S & Number of thiocarbonyl & 0.001 \\
\hline fr_amidine & Number of amidine groups & 0.001 \\
\hline fr_Al_OH_noTert & $\begin{array}{l}\text { Number of aliphatic hydroxyl groups excluding } \\
\text { tert-OH }\end{array}$ & 0.001 \\
\hline
\end{tabular}

Table S33. Feature importance for the regression model of PPI inhibitors targeting the XIAP / Smac complex.

\begin{tabular}{|l|l|r|}
\hline Feature & Description & Importance \\
\hline fr_Nhpyrrole & Number of H-pyrrole nitrogens & 0.120 \\
\hline Acceptor:Hydrophobe-5.00 & Number of pairs of acceptor-hydrophobe & 0.056 \\
\hline
\end{tabular}




\begin{tabular}{|c|c|c|}
\hline & atoms within 5 bonds & \\
\hline SMR_VSA9 & $\begin{array}{l}\text { MOE-type descriptors, molar refractivity } \\
\text { and surface area contributions }\end{array}$ & 0.055 \\
\hline Acceptor:Donor-6.00 & $\begin{array}{l}\text { Number of pairs of acceptor-donor atoms } \\
\text { within } 6 \text { bonds }\end{array}$ & 0.054 \\
\hline Donor:Donor-3.00 & $\begin{array}{l}\text { Number of pairs of donor-donor atoms } \\
\text { within } 3 \text { bonds }\end{array}$ & 0.052 \\
\hline Donor:Donor-2.00 & $\begin{array}{l}\text { Number of pairs of donor-donor atoms } \\
\text { within } 2 \text { bonds }\end{array}$ & 0.050 \\
\hline NOCount & Number of Nitrogens and Oxygens & 0.044 \\
\hline Hydrophobe:Poslonizable-2.00 & $\begin{array}{l}\text { Number of pairs of hydrophobe- } \\
\text { poslonazable atoms within } 2 \text { bonds }\end{array}$ & 0.042 \\
\hline Aromatic:Aromatic-3.00 & $\begin{array}{l}\text { Number of pairs of aromatic-aromatic } \\
\text { atoms within } 3 \text { bonds }\end{array}$ & 0.040 \\
\hline Hydrophobe:Poslonizable-4.00 & $\begin{array}{l}\text { Number of pairs of hydrophobe- } \\
\text { poslonazable atoms within } 4 \text { bonds }\end{array}$ & 0.036 \\
\hline Aromatic:Aromatic-2.00 & $\begin{array}{l}\text { Number of pairs of aromatic-aromatic } \\
\text { atoms within } 2 \text { bonds }\end{array}$ & 0.031 \\
\hline Donor:Hydrophobe-3.00 & $\begin{array}{l}\text { Number of pairs of donor-hydrophobe } \\
\text { atoms within } 3 \text { bonds }\end{array}$ & 0.030 \\
\hline Donor:Hydrophobe-4.00 & $\begin{array}{l}\text { Number of pairs of donor-hydrophobe } \\
\text { atoms within } 4 \text { bonds }\end{array}$ & 0.027 \\
\hline Aromatic:Aromatic-1.00 & $\begin{array}{l}\text { Number of pairs of aromatic-aromatic } \\
\text { atoms within } 1 \text { bond }\end{array}$ & 0.026 \\
\hline SMR_VSA10 & $\begin{array}{l}\text { MOE-type descriptors, molar refractivity } \\
\text { and surface area contributions }\end{array}$ & 0.025 \\
\hline Aromatic:Donor-5.00 & $\begin{array}{l}\text { Number of pairs of aromatic-donor atoms } \\
\text { within } 5 \text { bonds }\end{array}$ & 0.021 \\
\hline Hydrophobe_Count & Number of hydrophobe atoms & 0.021 \\
\hline Acceptor:Aromatic-2.00 & $\begin{array}{l}\text { Number of pairs of acceptor-aromatic } \\
\text { atoms within } 2 \text { bonds }\end{array}$ & 0.021 \\
\hline fr_priamide & Number of primary amides & 0.020 \\
\hline Acceptor:Hydrophobe-4.00 & $\begin{array}{l}\text { Number of pairs of acceptor-hydrophobe } \\
\text { atoms within } 4 \text { bonds }\end{array}$ & 0.020 \\
\hline fr_amide & Number of amides & 0.018 \\
\hline Aromatic:Hydrophobe-2.00 & $\begin{array}{l}\text { Number of pairs of aromatic-hydrophobe } \\
\text { atoms within } 2 \text { bonds }\end{array}$ & 0.018 \\
\hline Hydrophobe:Hydrophobe- 1.00 & $\begin{array}{l}\text { Number of pairs of hydrophobe-hydrophobe } \\
\text { atoms within } 1 \text { bond }\end{array}$ & 0.018 \\
\hline Hydrophobe:Hydrophobe-3.00 & $\begin{array}{l}\text { Number of pairs of hydrophobe-hydrophobe } \\
\text { atoms within } 3 \text { bonds }\end{array}$ & 0.018 \\
\hline Aromatic_Count & Number of atoms in aromatic rings & 0.017 \\
\hline Acceptor:Hydrophobe-3.00 & $\begin{array}{l}\text { Number of pairs of acceptor-hydrophobe } \\
\text { atoms within } 3 \text { bonds }\end{array}$ & 0.016 \\
\hline Hydrophobe:Hydrophobe- 4.00 & $\begin{array}{l}\text { Number of pairs of hydrophobe atoms } \\
\text { within } 4 \text { bonds }\end{array}$ & 0.015 \\
\hline Aromatic:Hydrophobe-1.00 & $\begin{array}{l}\text { Number of pairs of aromatic-hydrophobe } \\
\text { atoms within } 1 \text { bond }\end{array}$ & 0.011 \\
\hline
\end{tabular}




\begin{tabular}{|l|l|r|}
\hline Acceptor:Poslonizable-2.00 & $\begin{array}{l}\text { Number of pairs of acceptor-poslonazable } \\
\text { atoms within 2 bonds }\end{array}$ & 0.011 \\
\hline Poslonizable_Count & Number of poslonazable atoms & 0.010 \\
\hline Poslonizable:Poslonizable-3.00 & $\begin{array}{l}\text { Number of pairs of poslonazable- } \\
\text { poslonazable atoms within 3 bonds }\end{array}$ & 0.008 \\
\hline Acceptor:Poslonizable-3.00 & $\begin{array}{l}\text { Number of pairs of acceptor-poslonazable } \\
\text { atoms within 3 bonds }\end{array}$ & 0.006 \\
\hline PEOE_VSA3 & $\begin{array}{l}\text { MOE-type descriptors, partial charges and } \\
\text { surface area contributions }\end{array}$ & 0.006 \\
\hline PEOE_VSA8 & $\begin{array}{l}\text { MOE-type descriptors, partial charges and } \\
\text { surface area contributions }\end{array}$ & 0.005 \\
\hline VSA_EState8 & $\begin{array}{l}\text { MOE-type descriptors, surface area } \\
\text { contributions and EState indices }\end{array}$ & 0.005 \\
\hline SlogP_VSA11 & $\begin{array}{l}\text { MOE-type descriptors, LogP and surface } \\
\text { area contributions }\end{array}$ & 0.005 \\
\hline fr_Ar_N & Number of aromatic nitrogens & 0.004 \\
\hline Acceptor:Hydrophobe-2.00 & $\begin{array}{l}\text { Number of pairs of acceptor-hydrophobe } \\
\text { atoms within 2 bonds }\end{array}$ & 0.004 \\
\hline Acceptor:Hydrophobe-1.00 & $\begin{array}{l}\text { Number of pairs of acceptor-hydrophobe } \\
\text { atoms within 1 bond }\end{array}$ & 0.004 \\
\hline Poslonizable:Poslonizable-6.00 & $\begin{array}{l}\text { Number of pairs of poslonazable- } \\
\text { poslonazable atoms within 6 bonds }\end{array}$ & 0.004 \\
\hline Donor:Hydrophobe-2.00 & $\begin{array}{l}\text { Number of pairs of donor-hydrophobe } \\
\text { atoms within 2 bonds }\end{array}$ & 0.002 \\
\hline Acceptor:Acceptor-3.00 & $\begin{array}{l}\text { Number of pairs of acceptor-acceptor } \\
\text { atoms within 3 bonds }\end{array}$ & $\begin{array}{l}\text { Number of pairs of acceptor-poslonazable } \\
\text { atoms within 4 bonds }\end{array}$ \\
\hline Acceptor:Poslonizable-4.00 & & 0.001 \\
\hline
\end{tabular}

Table S34. Feature importance for general predictive model of PPI inhibitors activity (IC50).

\begin{tabular}{|l|l|r|}
\hline Feature & Description & Importance \\
\hline BalabanJ & Balaban's connectivity topological index & 0.071 \\
\hline NumHeteroatoms & Number of heteroatoms & 0.057 \\
\hline fr_C_O & Number of carbonyl O & 0.046 \\
\hline Chi4v & Molecular connectivity index & 0.044 \\
\hline SlogP_VSA5 & $\begin{array}{l}\text { MOE-type descriptors, LogP and surface } \\
\text { area contributions }\end{array}$ & 0.04 \\
\hline fr_amide & Number of amides & 0.035 \\
\hline PEOE_VSA3 & $\begin{array}{l}\text { MOE-type descriptors, partial charges and } \\
\text { surface area contributions }\end{array}$ & 0.033 \\
\hline PEOE_VSA10 & $\begin{array}{l}\text { MOE-type descriptors, partial charges and } \\
\text { surface area contributions }\end{array}$ & 0.032 \\
\hline Acceptor:Aromatic-6.00 & $\begin{array}{l}\text { Number of pairs of aromatic-aromatic atoms } \\
\text { within 6 bonds }\end{array}$ & 0.031 \\
\hline Aromatic:Neglonizable-2.00 & $\begin{array}{l}\text { Number of pairs of aromatic-neglonazable } \\
\text { atoms within 2 bonds }\end{array}$ & 0.031 \\
\hline Hydrophobe:Hydrophobe-6.00 & Number of pairs of hydrophobe-hydrophobe & 0.029 \\
\hline
\end{tabular}




\begin{tabular}{|c|c|c|}
\hline & atoms within 6 bonds & \\
\hline Donor:Donor-6.00 & $\begin{array}{l}\text { Number of pairs of donor-donor atoms } \\
\text { within } 6 \text { bonds }\end{array}$ & 0.029 \\
\hline SMR_VSA7 & $\begin{array}{l}\text { MOE-type descriptors, molar refractivity and } \\
\text { surface area contributions }\end{array}$ & 0.029 \\
\hline SlogP_VSA10 & $\begin{array}{l}\text { MOE-type descriptors, LogP and surface } \\
\text { area contributions }\end{array}$ & 0.028 \\
\hline Donor_Count & Number of hydrogen donors & 0.028 \\
\hline PEOE_VSA8 & $\begin{array}{l}\text { MOE-type descriptors, partial charges and } \\
\text { surface area contributions }\end{array}$ & 0.028 \\
\hline fr_NHO & Number of Tertiary amines & 0.027 \\
\hline Hydrophobe_Count & Number of hydrophobe atoms & 0.027 \\
\hline Hydrophobe:Hydrophobe- 5.00 & $\begin{array}{l}\text { Number of pairs of hydrophobe-hydrophobe } \\
\text { atoms within } 5 \text { bonds }\end{array}$ & 0.027 \\
\hline fr_bicyclic & Number of bicyclic structures & 0.023 \\
\hline SlogP_VSA12 & $\begin{array}{l}\text { MOE-type descriptors, LogP and surface } \\
\text { area contributions }\end{array}$ & 0.022 \\
\hline Aromatic:Aromatic-3.00 & $\begin{array}{l}\text { Number of pairs of aromatic-aromatic atoms } \\
\text { within } 3 \text { bonds }\end{array}$ & 0.02 \\
\hline Aromatic:Aromatic-6.00 & $\begin{array}{l}\text { Number of pairs of aromatic-aromatic atoms } \\
\text { within } 6 \text { bonds }\end{array}$ & 0.02 \\
\hline Poslonizable:Poslonizable-2.00 & $\begin{array}{l}\text { Number of pairs of poslonazable- } \\
\text { poslonazable atoms within } 2 \text { bonds }\end{array}$ & 0.019 \\
\hline fr_methoxy & Number of methoxy groups $-\mathrm{OCH} 3$ & 0.019 \\
\hline Aromatic:Aromatic-5.00 & $\begin{array}{l}\text { Number of pairs of aromatic-aromatic atoms } \\
\text { within } 5 \text { bonds }\end{array}$ & 0.019 \\
\hline SlogP_VSA8 & $\begin{array}{l}\text { MOE-type descriptors, LogP and surface } \\
\text { area contributions }\end{array}$ & 0.018 \\
\hline fr_amidine & Number of amidine groups & 0.018 \\
\hline fr_unbrch_alkane & \begin{tabular}{|l|} 
Number of unbranched alkanes of at least 4 \\
members (excludes halogenated alkanes)
\end{tabular} & 0.017 \\
\hline Donor:Poslonizable-6.00 & $\begin{array}{l}\text { Number of pairs of donor-poslonazable } \\
\text { atoms within } 6 \text { bonds }\end{array}$ & 0.014 \\
\hline Hydrophobe:Poslonizable-4.00 & $\begin{array}{l}\text { Number of pairs of hydrophobe- } \\
\text { poslonazable atoms within } 4 \text { bonds }\end{array}$ & 0.013 \\
\hline fr_phenol & Number of phenols & 0.013 \\
\hline fr_ketone & Number of ketones & 0.013 \\
\hline Hydrophobe:Poslonizable- 6.00 & $\begin{array}{l}\text { Number of pairs of hydrophobe- } \\
\text { poslonazable atoms within } 6 \text { bonds }\end{array}$ & 0.012 \\
\hline fr_ester & Number of esters & 0.011 \\
\hline fr_phos_ester & Number of phosphoric ester groups & 0.009 \\
\hline fr_guanido & Number of guanidine groups & 0.009 \\
\hline fr_sulfide & Number of thioether & 0.007 \\
\hline Aromatic:Poslonizable-6.00 & $\begin{array}{l}\text { Number of pairs of aromatic-poslonazable } \\
\text { atoms within } 6 \text { bonds }\end{array}$ & 0.007 \\
\hline Aromatic:Poslonizable-4.00 & $\begin{array}{l}\text { Number of pairs of aromatic-poslonazable } \\
\text { atoms within } 4 \text { bonds }\end{array}$ & 0.006 \\
\hline
\end{tabular}




\begin{tabular}{|l|l|r|}
\hline fr_phos_acid & Number of phosphoric acid groups & 0.006 \\
\hline Donor:Poslonizable-3.00 & $\begin{array}{l}\text { Number of pairs of donor-poslonazable } \\
\text { atoms within 3 bonds }\end{array}$ & 0.006 \\
\hline Donor:Poslonizable-2.00 & $\begin{array}{l}\text { Number of pairs of donor-poslonazable } \\
\text { atoms within 2 bonds }\end{array}$ & 0.006 \\
\hline fr_term_acetylene & Number of terminal acetylenes & 0.002 \\
\hline
\end{tabular}

Table S35. Distribution of PPIs inhibitors retrieved from 3 databases: TIMBAL, iPPI-DB, 2P2I-DB. Number of compounds per PPI target is shown before and after clustering with Tanimoto similarity cutoff of 0.8 .

\begin{tabular}{|c|c|c|}
\hline PPI & \# compounds & \# compounds (clustering 0.8 ) \\
\hline Integrins & 1606 & 907 \\
\hline Bromodomain / Histone & 680 & 501 \\
\hline MDM2-Like / P53 & 768 & 440 \\
\hline LFA / ICAM & 277 & 150 \\
\hline BCL2-Like / BAX-BAK & 386 & 149 \\
\hline LEDGF / IN & 158 & 122 \\
\hline HIF-1a & 121 & 82 \\
\hline Cyclophilins & 105 & 73 \\
\hline XIAP / Smac & 99 & 68 \\
\hline Ras / SOS1 & 64 & 55 \\
\hline WDR5/MLL & 39 & 37 \\
\hline CD80 / CD28 & 73 & 35 \\
\hline BRD2 / Ack & 47 & 32 \\
\hline MENIN / MLL & 49 & 32 \\
\hline Annexin A2/S100-A10 & 44 & 29 \\
\hline Keap1 / Nrf2 & 31 & 26 \\
\hline Neuropilin / VEGF & 41 & 24 \\
\hline FKBP1A/FK506 & 29 & 21 \\
\hline STAT3 & 38 & 21 \\
\hline IL2 / IL-2R & 36 & 18 \\
\hline TTR & 19 & 17 \\
\hline VHL / HIF1-alpha & 33 & 17 \\
\hline Transthyretin & 16 & 16 \\
\hline BRD4 / NUT & 16 & 15 \\
\hline BetaCatenin / Tcf4 and Tcf3 & 40 & 15 \\
\hline Rac1 & 15 & 15 \\
\hline Tubulin & 14 & 14 \\
\hline SPIN1 / H3 & 15 & 10 \\
\hline CIAP1-BIR3/CASPASE-9 & 10 & 9 \\
\hline E2 / E1 & 11 & 9 \\
\hline MLLT1 / H3 & 16 & 8 \\
\hline
\end{tabular}




\begin{tabular}{|l|r|r|}
\hline DCN1/UBC12 & 7 & 7 \\
\hline PCNA trimer & 7 & 7 \\
\hline C-Myc / Max & 8 & 7 \\
\hline SOD1 & 4 & 7 \\
\hline RUNX1 / CBFb & 5 & 5 \\
\hline TNFa / TNFa & 4 & 4 \\
\hline VEGF / VEGFR & 4 & 4 \\
\hline ZipA / ftsZ & 3 & 4 \\
\hline 53BP1 / H4 & 3 & 4 \\
\hline WD40 / H3 & 2 & 3 \\
\hline CRM1 / Rev & 2 & 3 \\
\hline NRP / VEGF & 3 & 2 \\
\hline UPAR / UPA & 1 & 2 \\
\hline BRI1 & 1 & 2 \\
\hline CD4 / gp120 & 1 & 1 \\
\hline CD40 / CD154 & 1 & 1 \\
\hline CaM / CaMBD2 & 1 & 1 \\
\hline Rad51 & 1 & 1 \\
\hline TNFR1A / TNFB & 1 & 1 \\
\hline Tak1 / Tab1 & & 1 \\
\hline
\end{tabular}




\section{FIGURES}
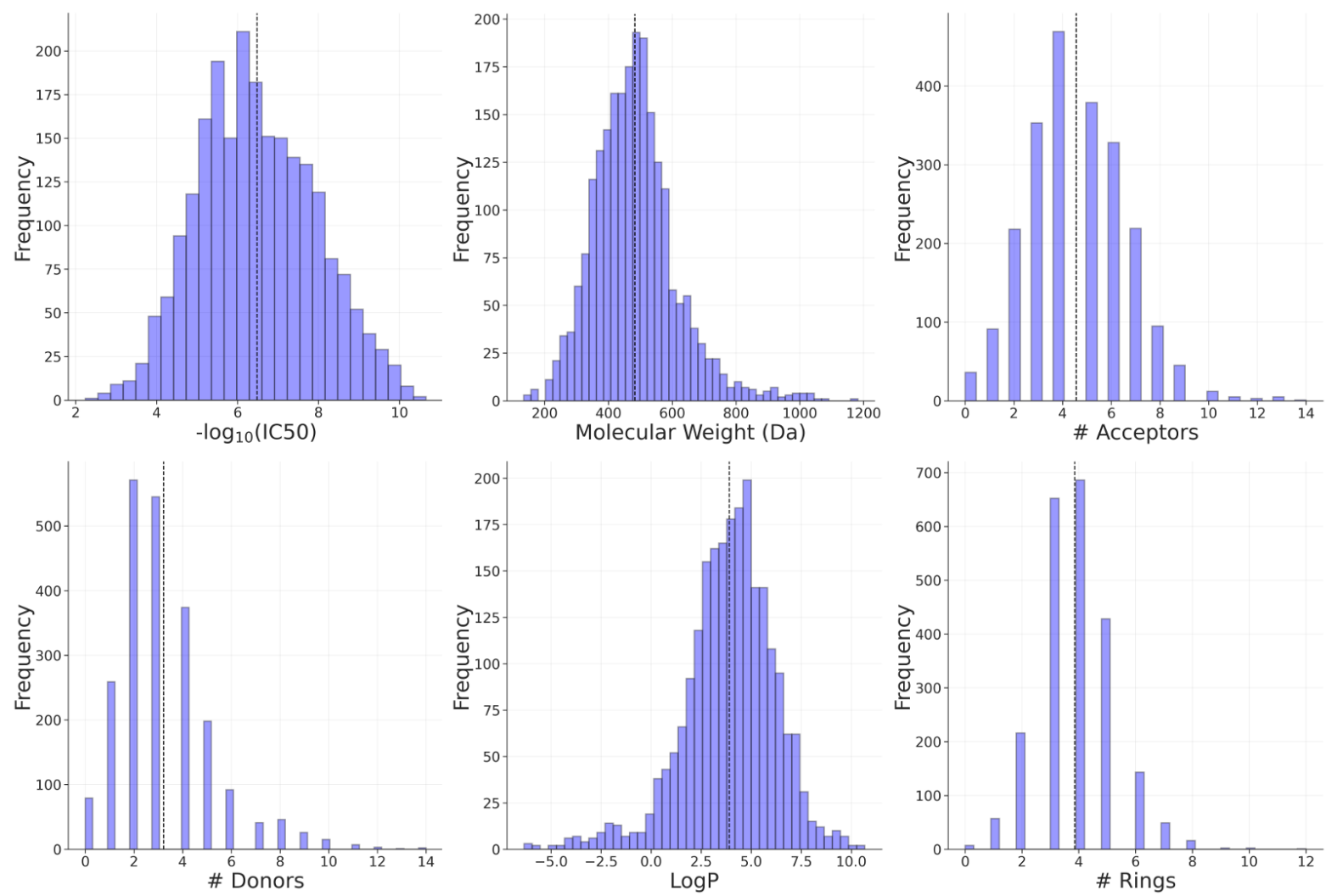

Figure S1 - Distribution of experimental IC50 values and property distribution of PPI inhibitors. The topleft histogram shows the distribution of experimental IC50 values for all inhibitors in the dataset after clustering with Tanimoto similarity of 0.8 , in terms of $-\log _{10}$ (Molar). The remaining histograms depict the distribution of common physicochemical properties of the compounds, including molecular weight (in $\mathrm{Da}$ ), log $\mathrm{P}$, number of hydrogen acceptors and donors, and number of rings.
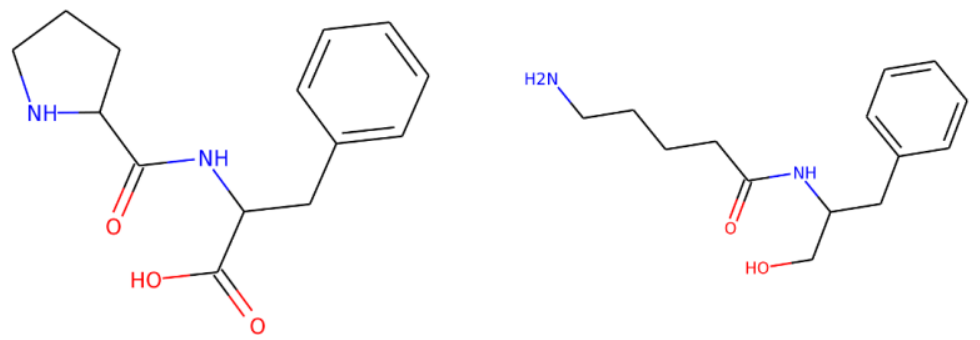<smiles>CC(=O)N1CCCC(C(=O)NCCC(=O)O)C1</smiles><smiles>CCNC(C)Cc1ccc(-c2c(OC)cccc2OC)cc1</smiles><smiles>C=C(O)CCNC(=O)CCC(CC)C(=O)CCC1CCCCC1</smiles> 
Figure S2. Frequent substructures within the dataset of PPI inhibitors. More potent (IC50<1uM) compounds are enriched with ring substructures, including biphenyls.
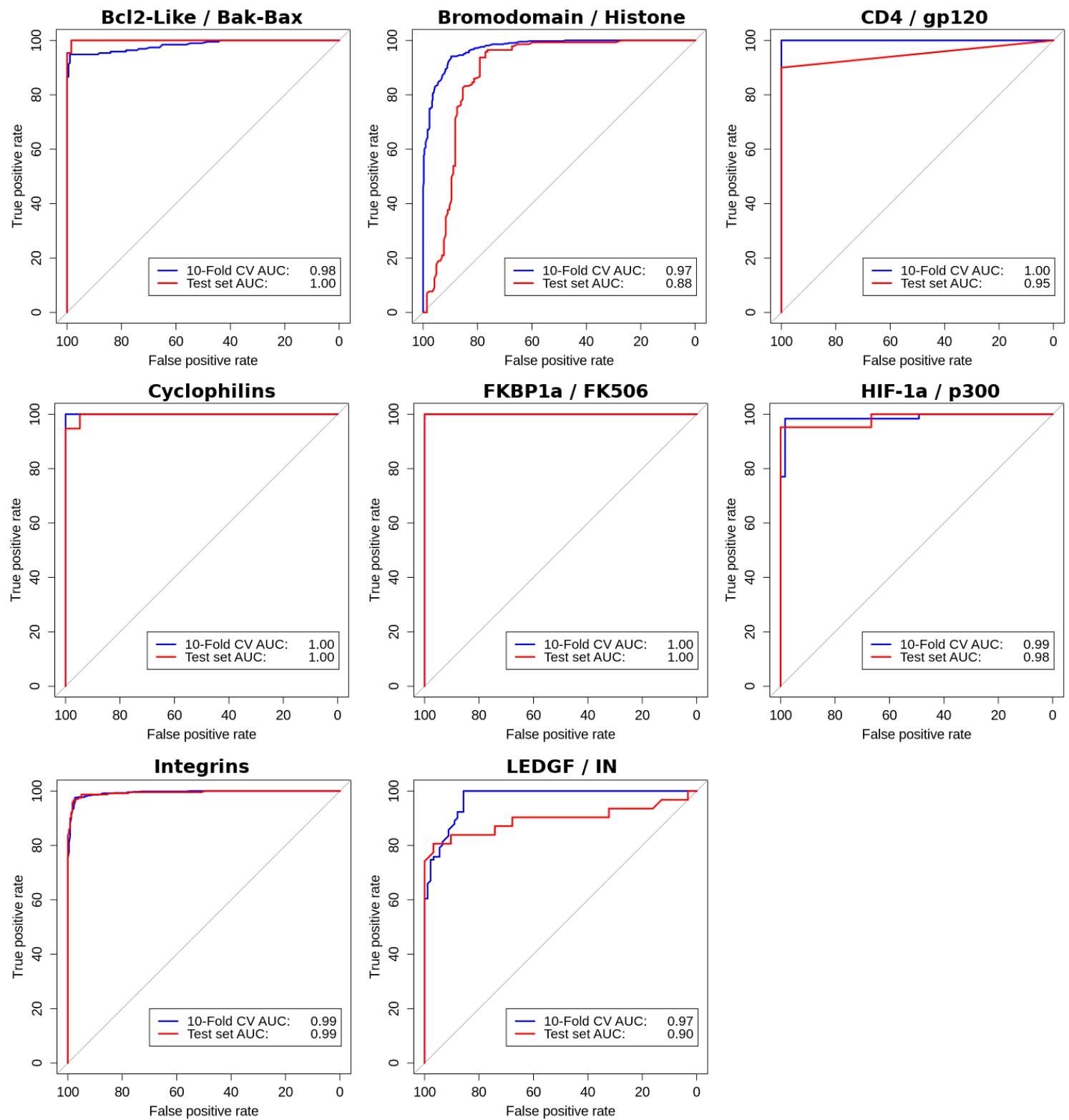

Figure S3. ROC curves for class-specific predictors during training under 10-Fold cross-validation and non-redundant test sets. 

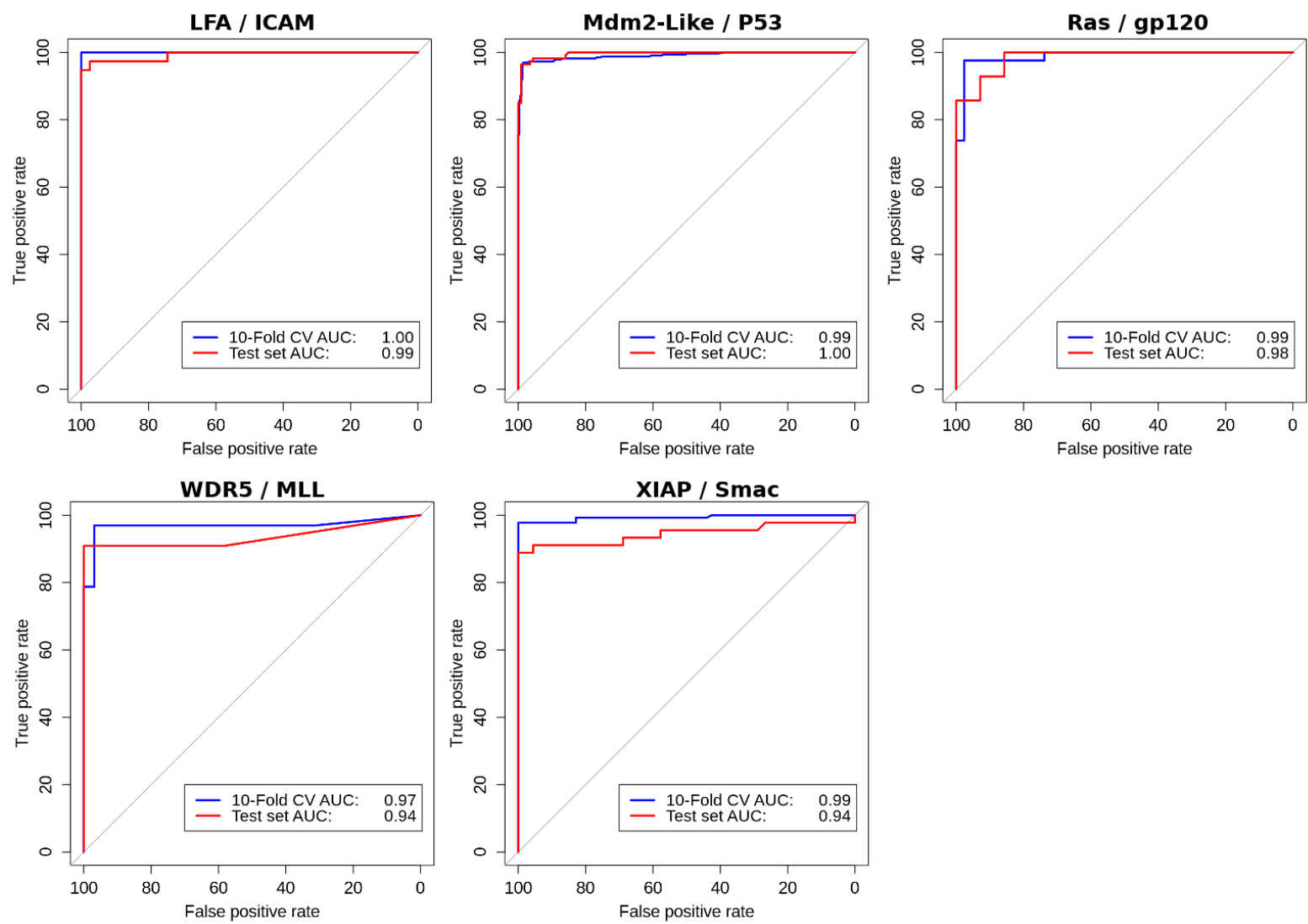

Figure S4. ROC curves for class-specific predictors on 10-Fold cross-validation and non-redundant test sets. 

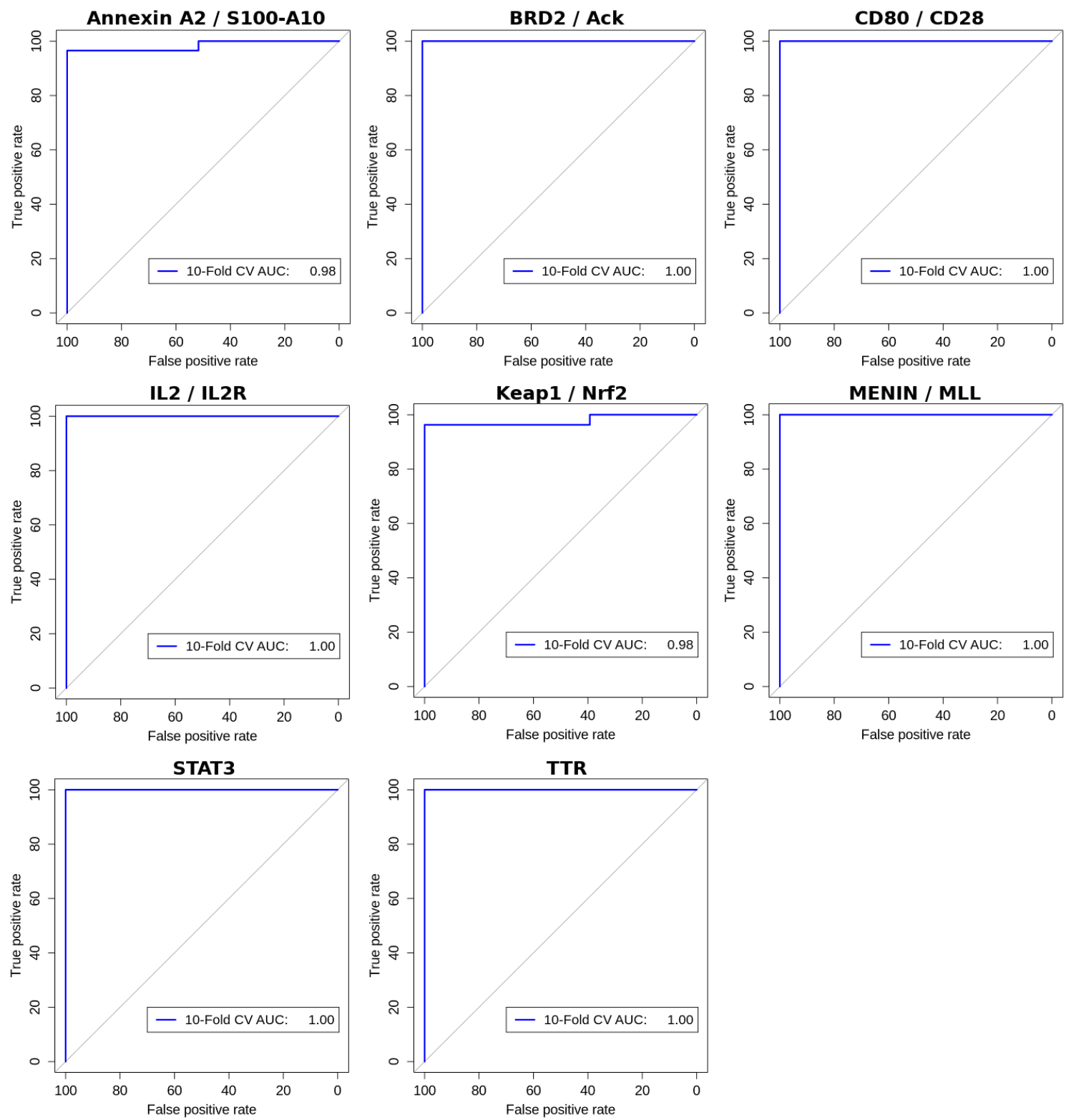

Figure S5. ROC curves for class-specific predictors with a limited number of inhibitors. Given the lack of data available for $8 \mathrm{PPI}$ targets results are shown under 10-Fold cross-validation. 

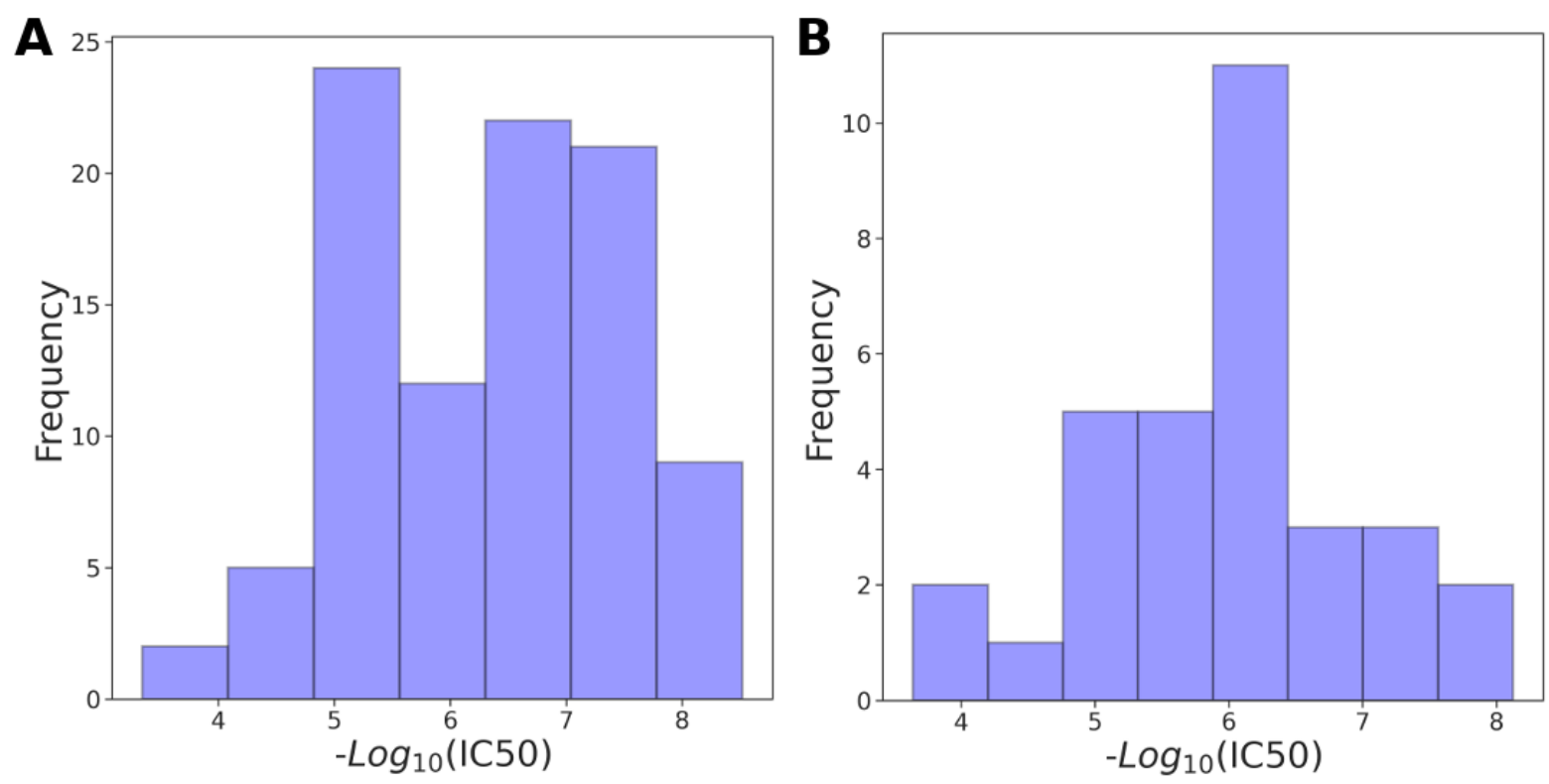

Figure S6. Distribution of PPI half maximal inhibitory concentration (IC50) for inhibitors targeting the BCL2-Like / BAX-BAK complex. A) depicts distribution on the set of molecules used for training the regression model and $B$ ) on the non-redundant test set. IC50 values are shown as - $\log 10$.
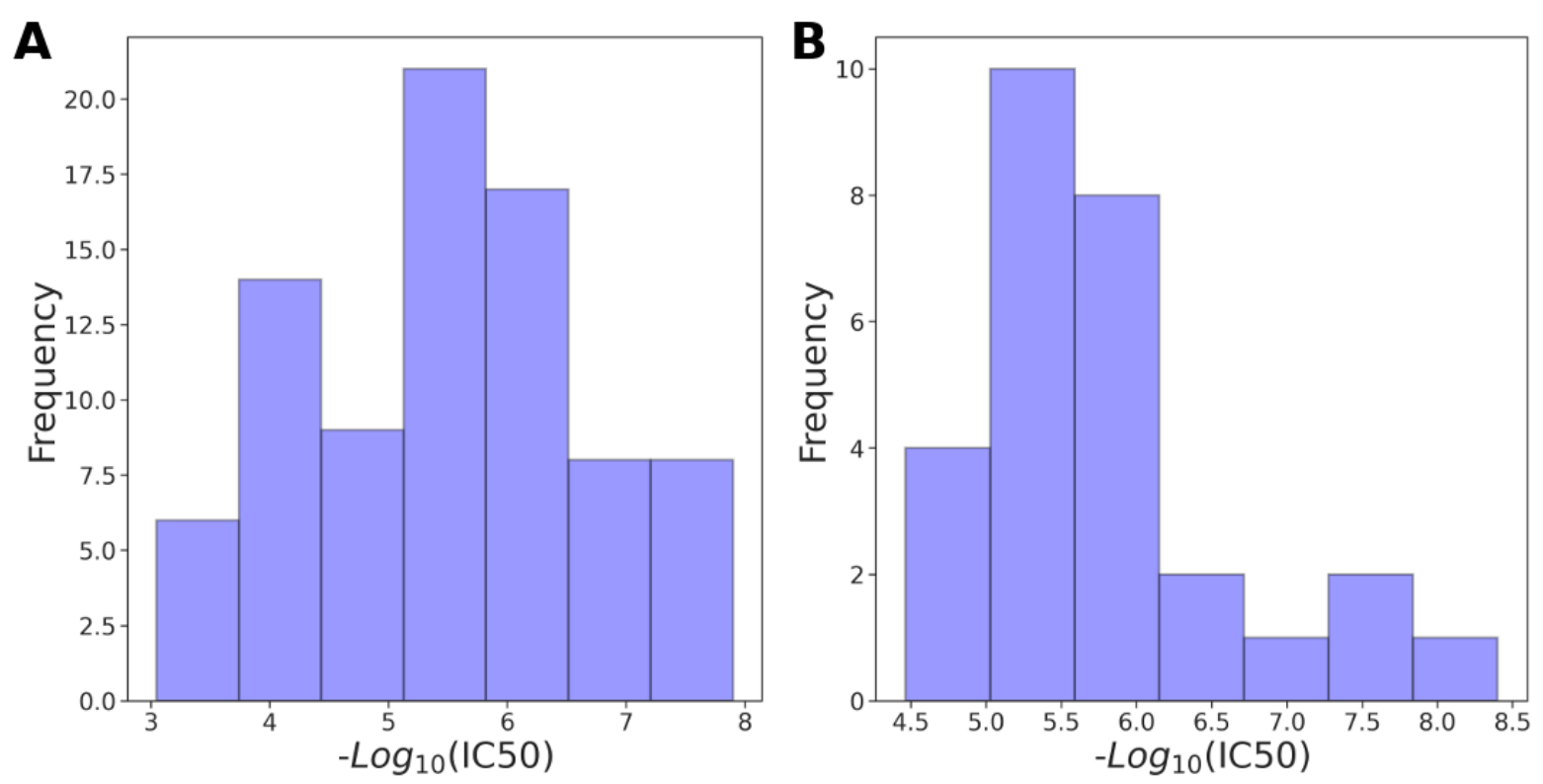

Figure S7. Distribution of PPI half maximal inhibitory concentration (IC50) for inhibitors targeting the Bromodomain / Histone complex. A) depicts distribution on the set of molecules used for training the regression model and $B$ ) on the non-redundant test set. IC50 values are shown as - $\log 10$. 

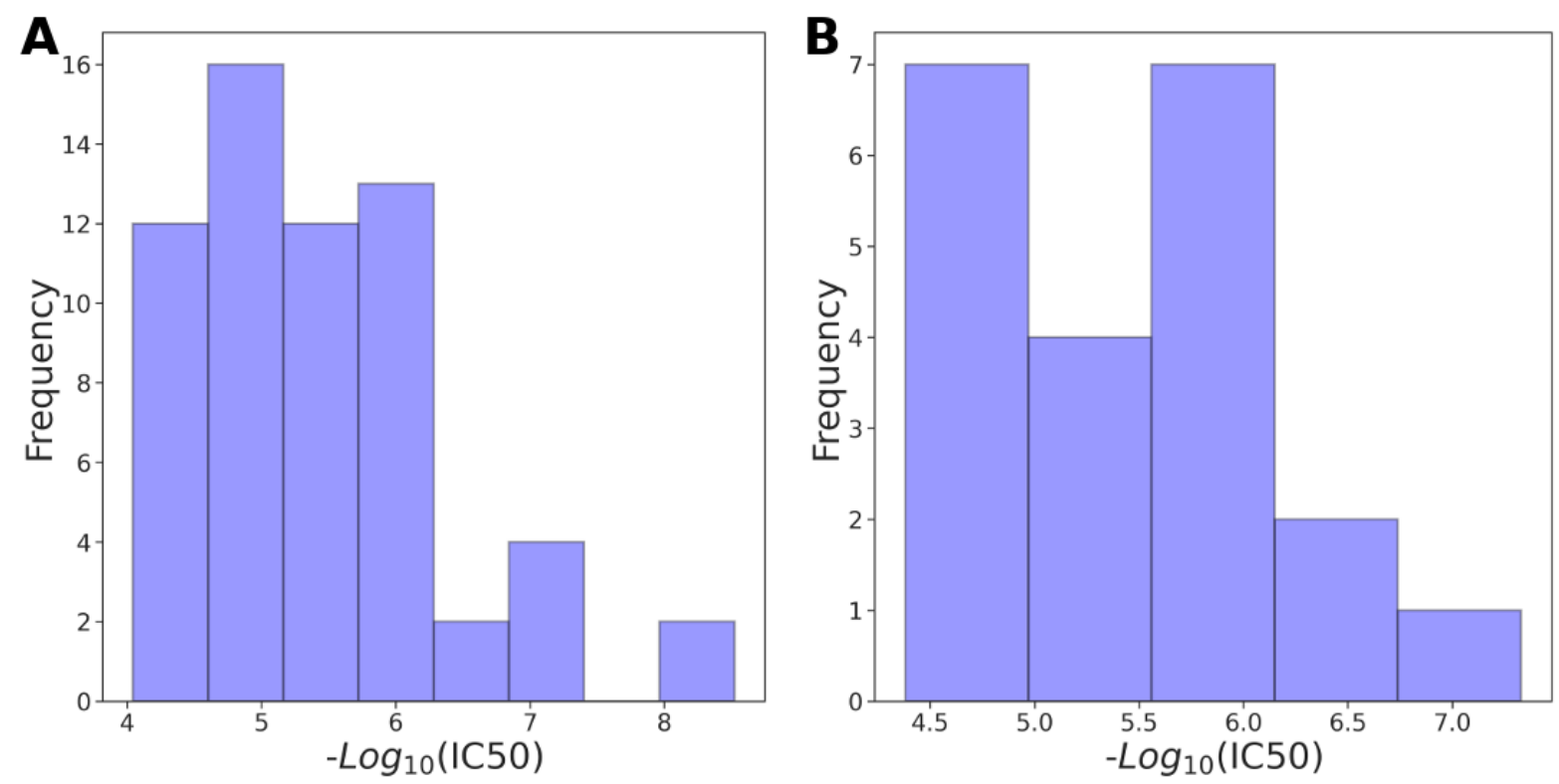

Figure S8. Distribution of PPI half maximal inhibitory concentration (IC50) for inhibitors targeting the HIF-1a / p300 complex. A) depicts distribution on the set of molecules used for training the regression model and B) on the non-redundant test set. IC50 values are shown as - $\log 10$.
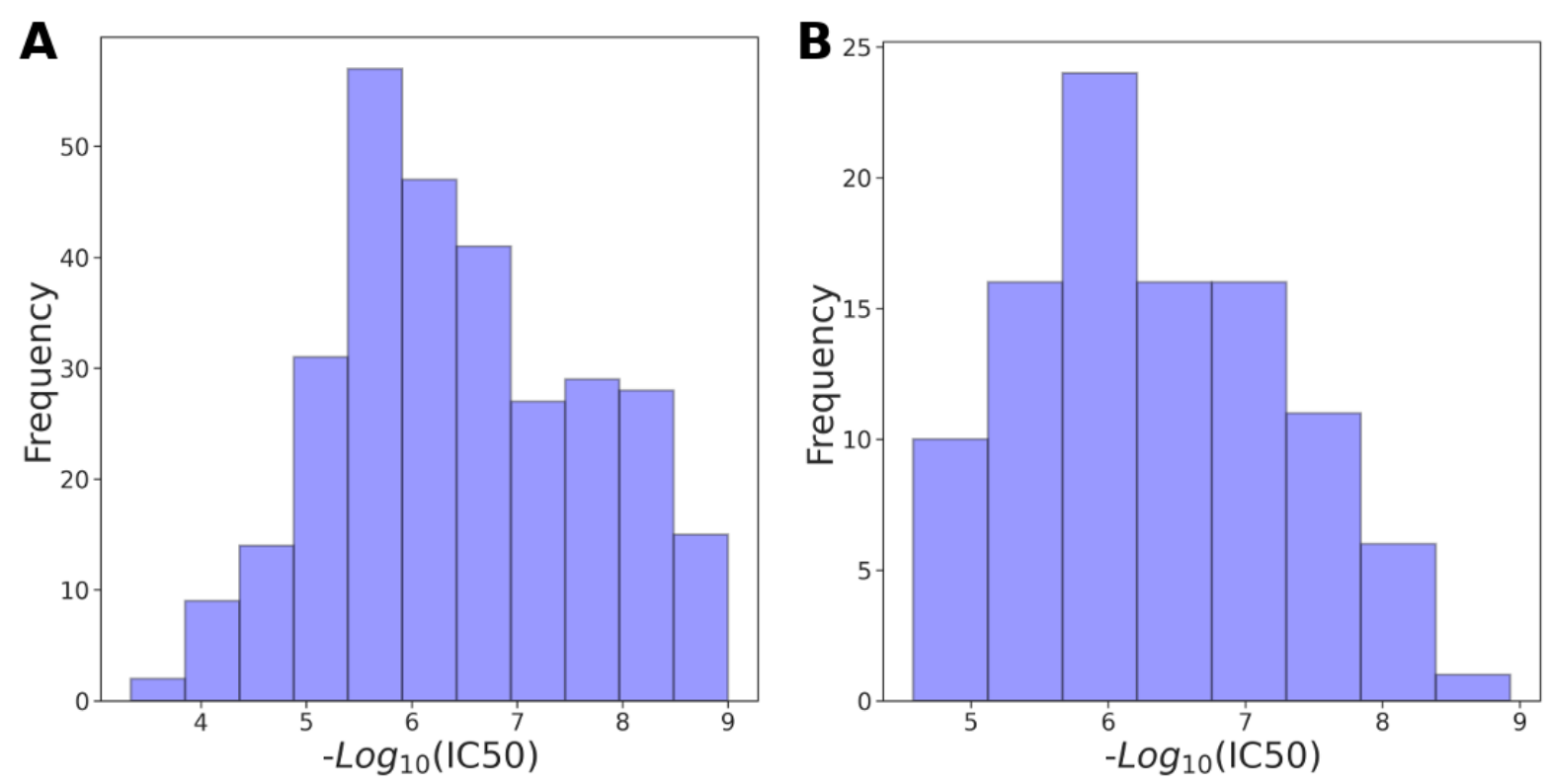

Figure S9. Distribution of PPI half maximal inhibitory concentration (IC50) for inhibitors targeting the Mdm2-Like / P53 complex. A) depicts distribution on the set of molecules used for training the regression model and B) on the non-redundant test set. IC50 values are shown as - $\log 10$. 

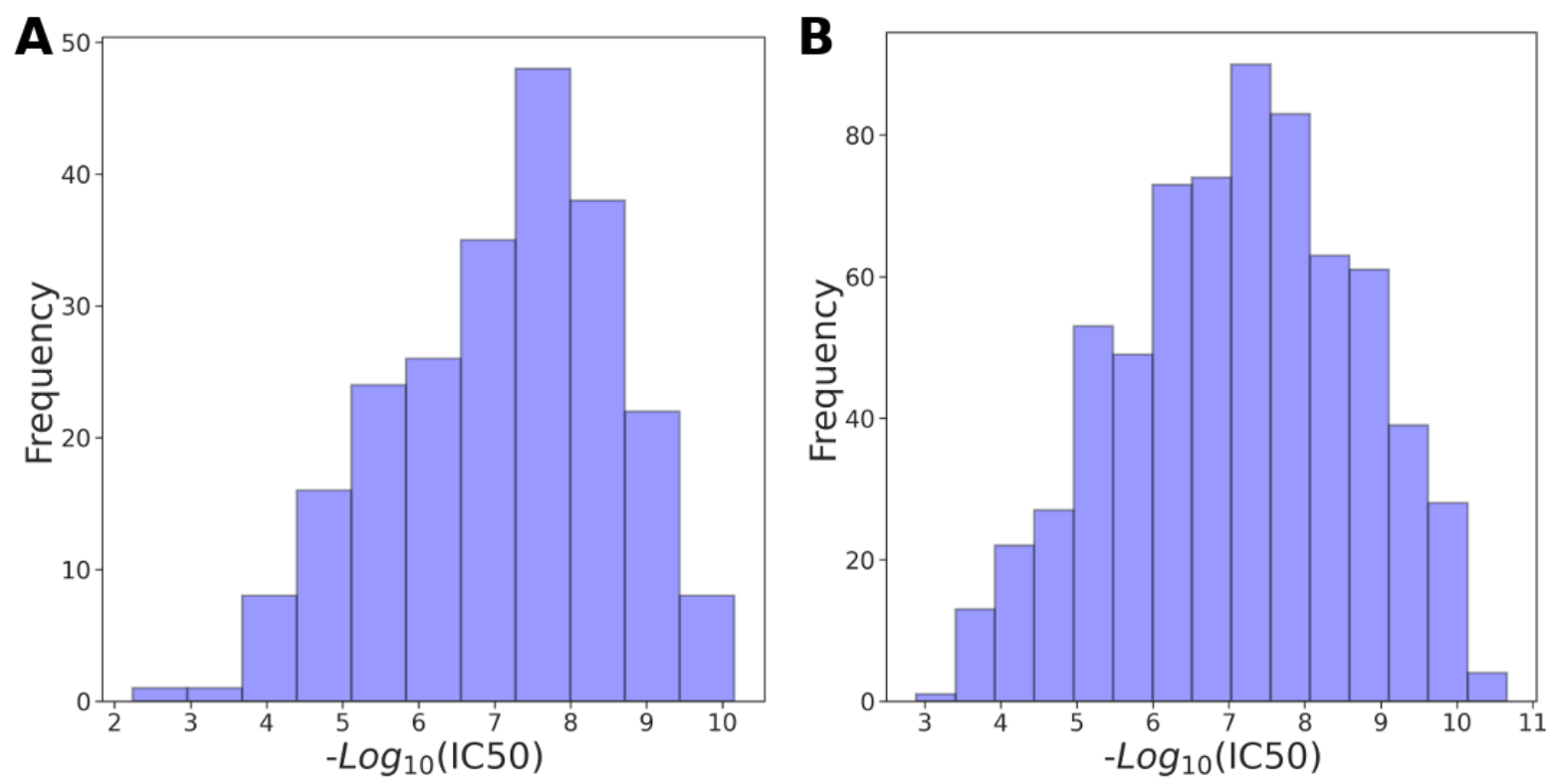

Figure S10. Distribution of PPI half maximal inhibitory concentration (IC50) for inhibitors targeting the Integrins complex. A) depicts distribution on the set of molecules used for training the regression model and $B$ ) on the non-redundant test set. IC50 values are shown as - $\log 10$.
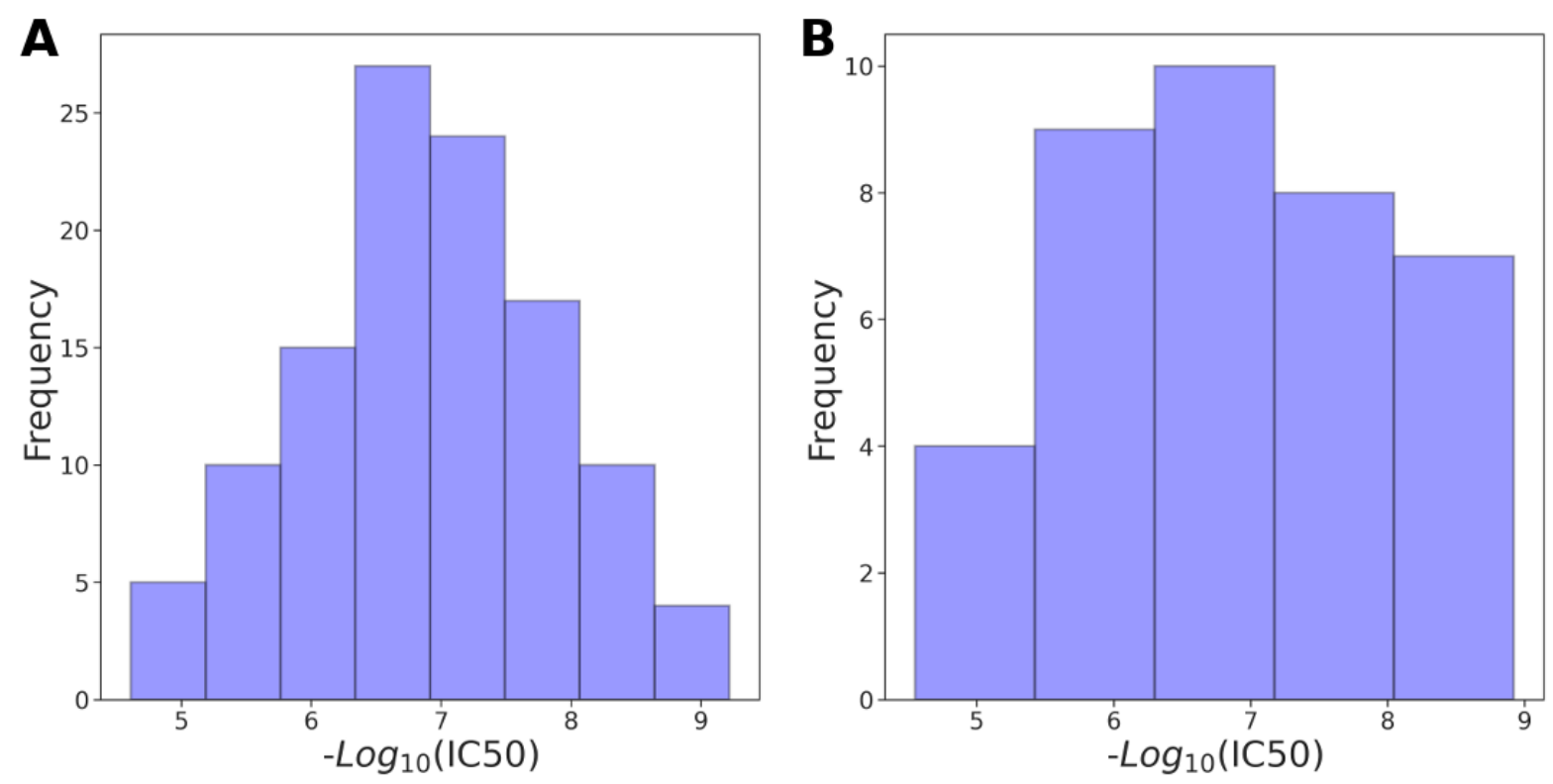

Figure S11. Distribution of PPI half maximal inhibitory concentration (IC50) for inhibitors targeting the LFA / ICAM complex. A) depicts distribution on the set of molecules used for training the regression model and B) on the non-redundant test set. IC50 values are shown as - $\log 10$. 

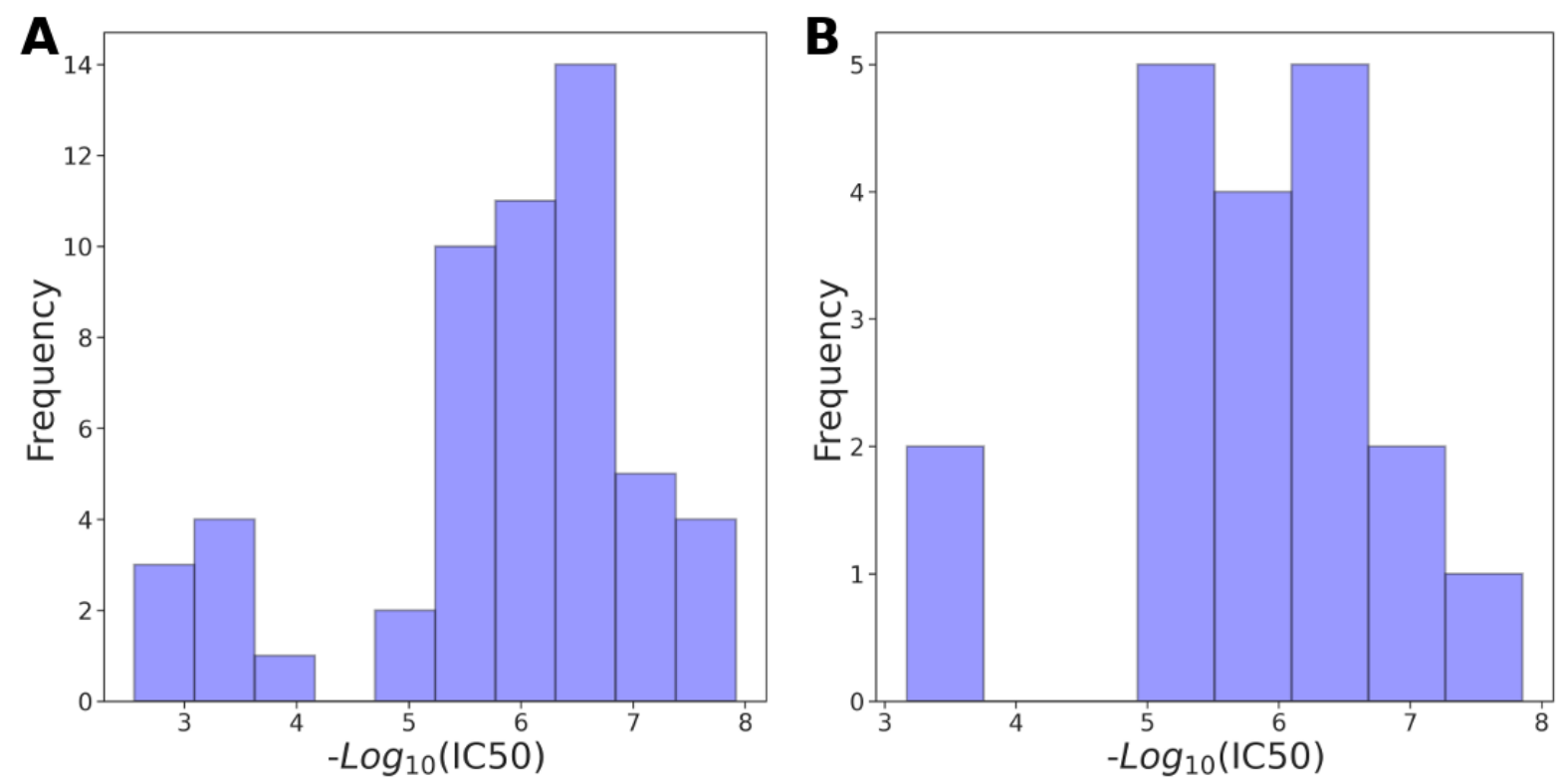

Figure S12. Distribution of PPI half maximal inhibitory concentration (IC50) for inhibitors targeting the Cyclophilins complex. A) depicts distribution on the set of molecules used for training the regression model and B) on the non-redundant test set. IC50 values are shown as - $\log 10$.
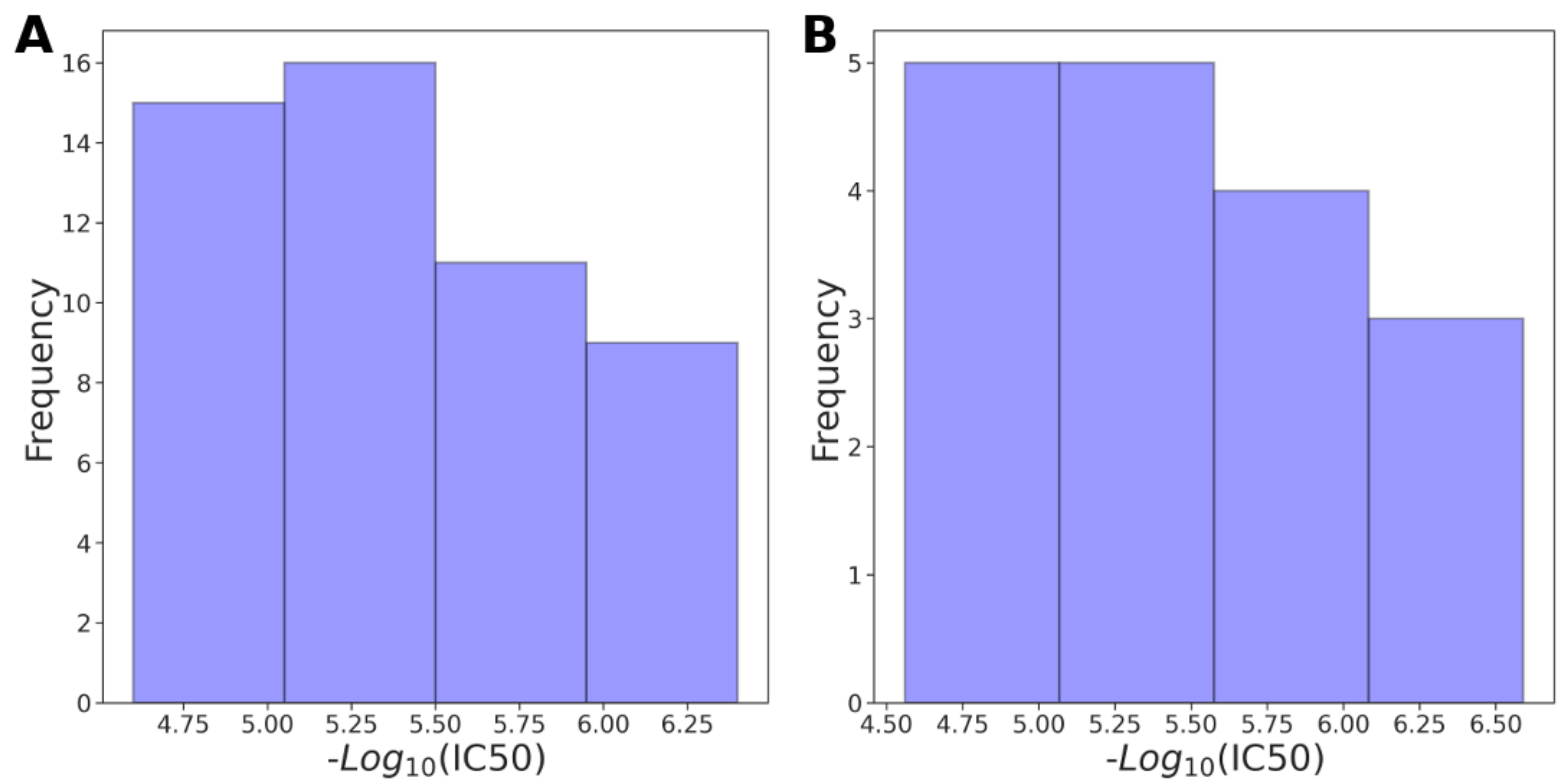

Figure S13. Distribution of PPI half maximal inhibitory concentration (IC50) for inhibitors targeting the LEDGF / IN complex. A) depicts distribution on the set of molecules used for training the regression model and B) on the non-redundant test set. IC50 values are shown as - $\log 10$. 

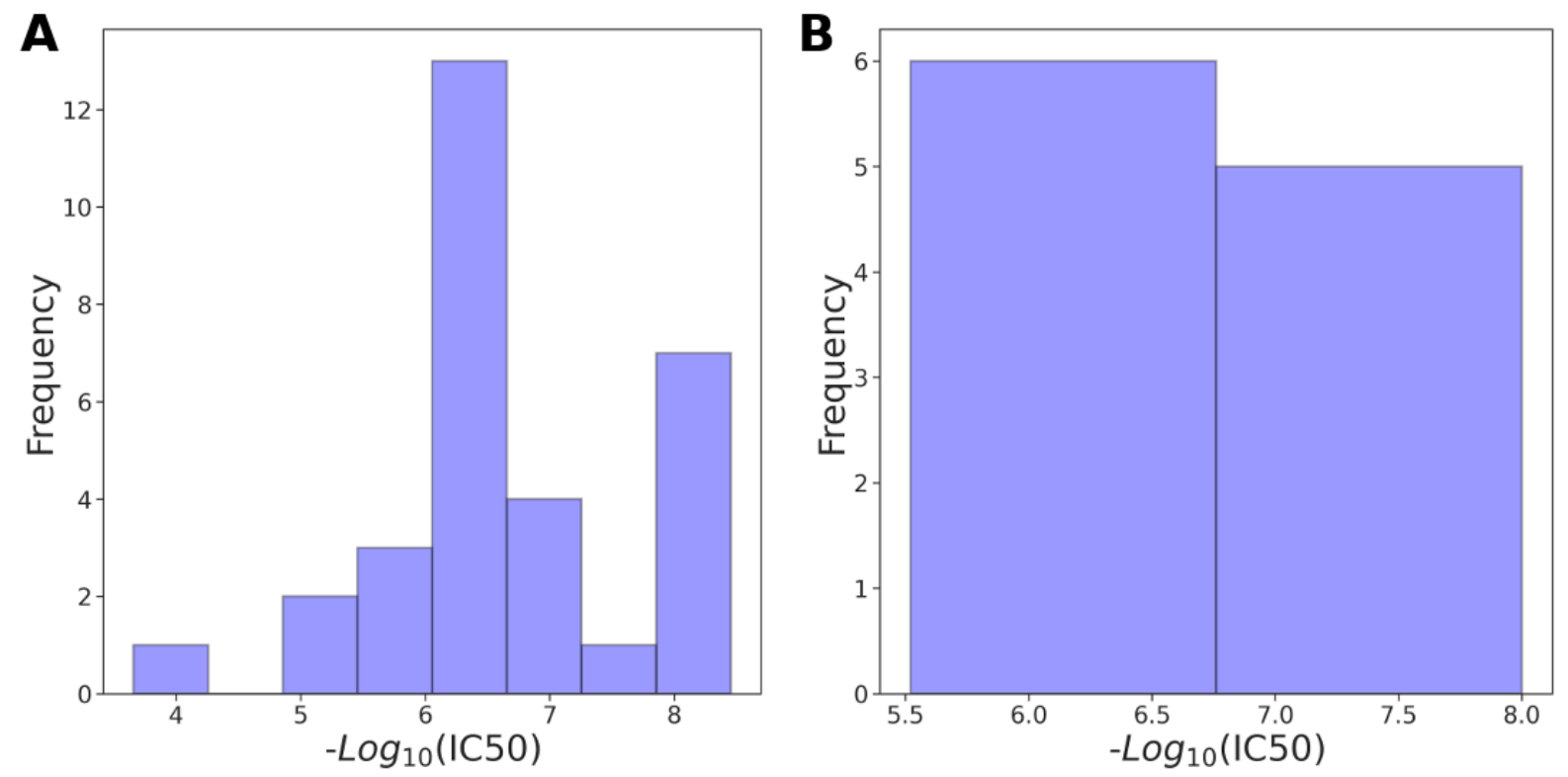

Figure S14. Distribution of PPI half maximal inhibitory concentration (IC50) for inhibitors targeting the XIAP / Smac complex. A) depicts distribution on the set of molecules used for training the regression model and B) on the non-redundant test set. IC50 values are shown as - $\log 10$.
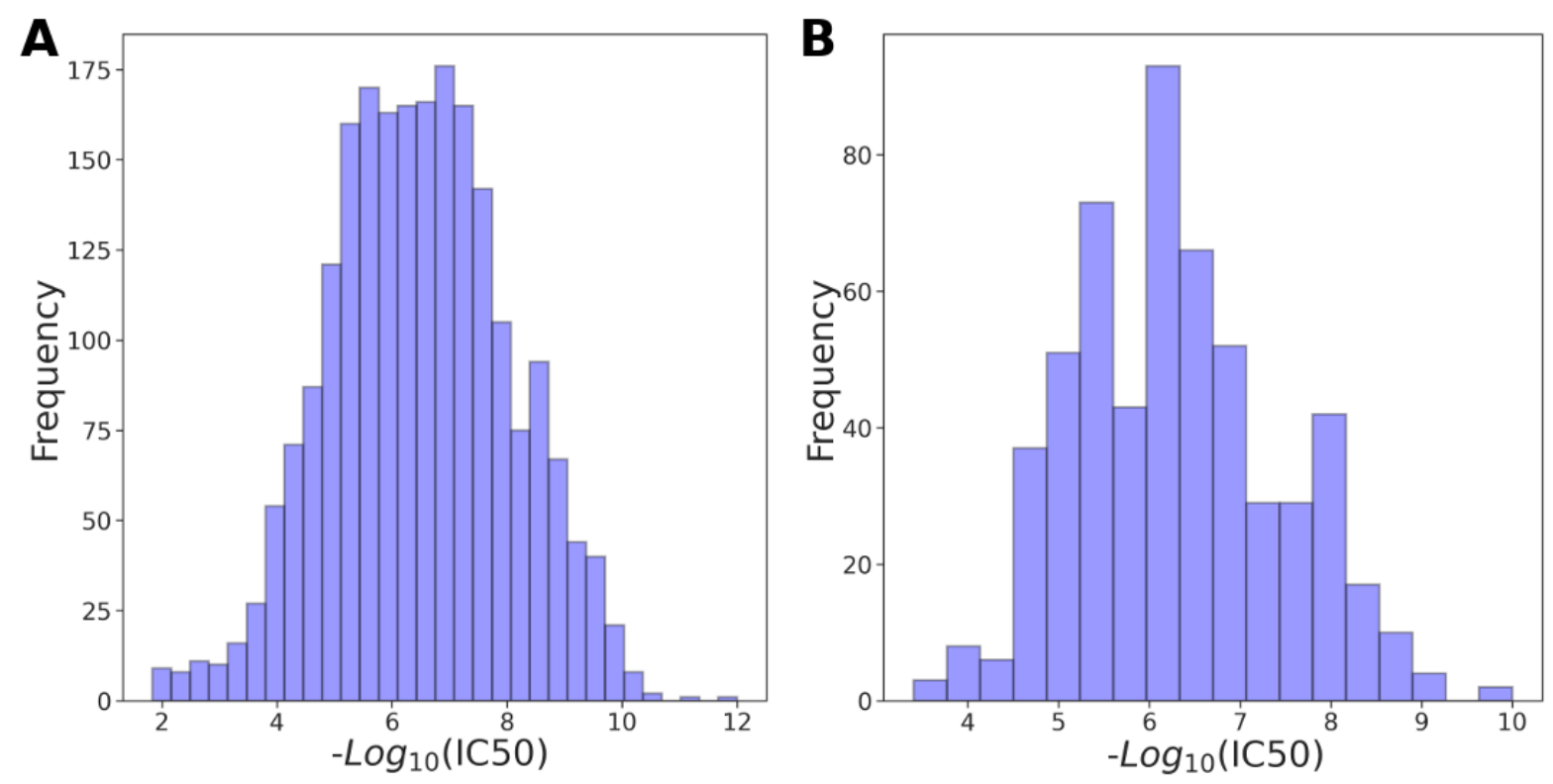

Figure S15. Distribution of PPI half maximal inhibitory concentration (IC50) for inhibitors used to build the general predictor of PPI inhibitory activity. A) depicts distribution on the set of molecules used for training the regression model and B) on the non-redundant test set. IC50 values are shown as - $\log 10$. 


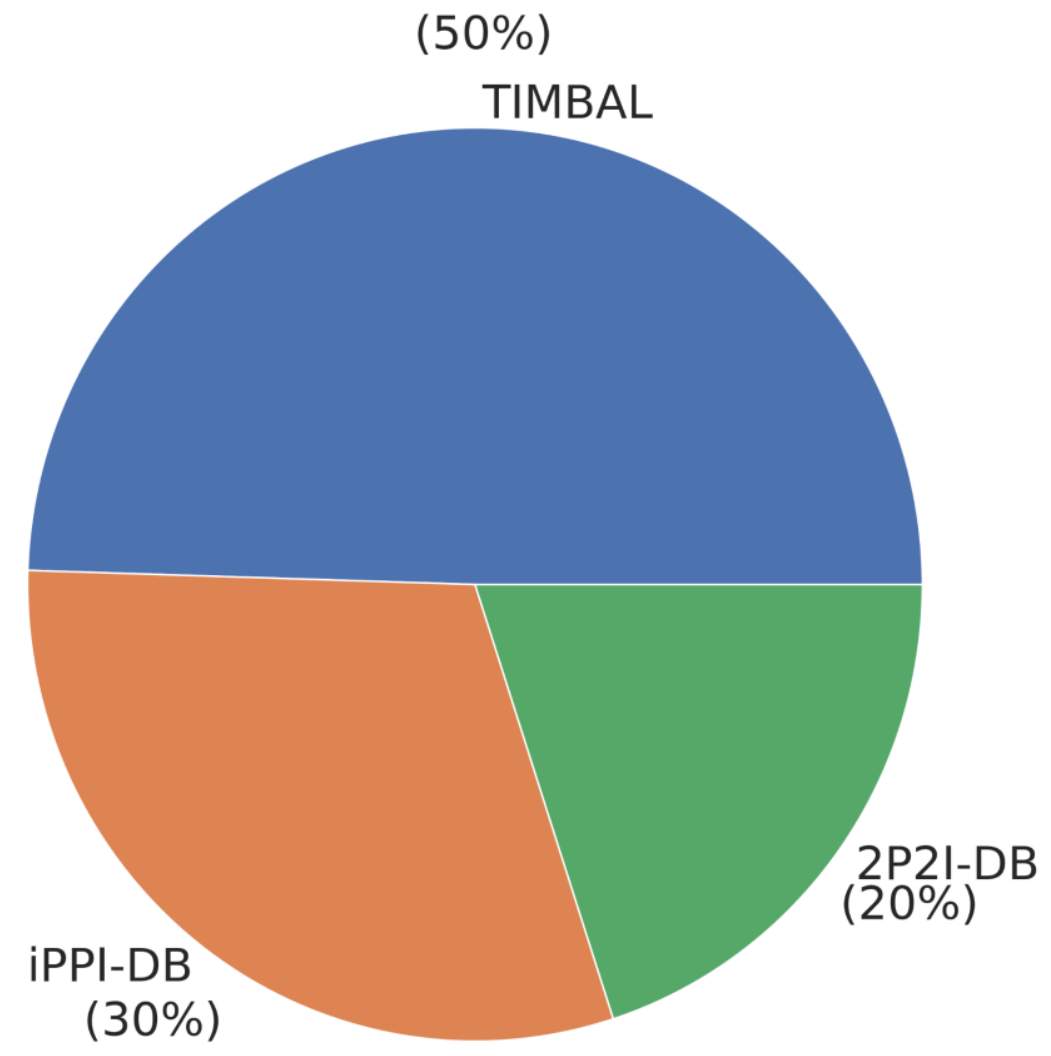

Figure S16. Distribution of PPI inhibitors retrieved from 3 different databases: TIMBAL, IPPI-DB and 2P2I-DB. 